\title{
The BepiColombo Laser Altimeter
}

\author{
N. Thomas ${ }^{1}$ - H. Hussmann ${ }^{2}$ T. Spohn ${ }^{2,3}$ - L.M. Lara ${ }^{4}$ U. Christensen ${ }^{5}$ M. Affolter ${ }^{1}$. \\ T. Bandy ${ }^{1}$ T. Beck ${ }^{1}$ S. Chakraborty ${ }^{1}$ U. Geissbuehler ${ }^{1} \cdot$ M. Gerber ${ }^{1} \cdot$ K. Ghose $^{1}$. \\ J. Gouman ${ }^{1}$ - S. HosseiniArani ${ }^{1}$ - K. Kuske ${ }^{1}$ - A. Peteut ${ }^{1}$ • D. Piazza ${ }^{1}$ - M. Rieder ${ }^{1}$. \\ A. Servonet ${ }^{1}$ - C. Althaus ${ }^{2}$ - T. Behnke ${ }^{2}$ - K. Gwinner ${ }^{2}$ - C. Hüttig ${ }^{2}$. R. Kallenbach ${ }^{2,5}$. \\ A. Lichopoj ${ }^{2}$ - K. Lingenauber ${ }^{2}$ - H.-G. Lötzke ${ }^{2}$ - F. Lüdicke ${ }^{2}$ - H. Michaelis ${ }^{2}$. \\ J. Oberst $^{2} \cdot$ R. Schrödter ${ }^{2}$ - A. Stark ${ }^{2}$ - G. Steinbrügge ${ }^{2,6} \cdot$ S. del Togno ${ }^{2}$. \\ K. Wickhusen ${ }^{2}$ J.M. Castro ${ }^{4}$ M. Herranz ${ }^{4}$ J. Rodrigo ${ }^{4}$ - H. Perplies ${ }^{5}$ - T. Weigel $^{7}$. \\ S. Schulze-Walewski ${ }^{7}$ - S. Blum ${ }^{7}$ - A. Casciello ${ }^{7}$ - E. Rugi-Grond ${ }^{7} \cdot$ W. Coppoolse ${ }^{7}$.

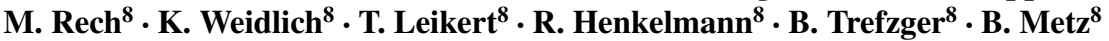

Received: 17 June 2020 / Accepted: 9 January 2021 / Published online: 15 February 2021

(C) The Author(s) 2021

\begin{abstract}
The BepiColombo Laser Altimeter (BELA) is the first European laser altimeter constructed for interplanetary flight. BELA uses a $50 \mathrm{~mJ}$ pulsed Nd:YAG laser operating at $10 \mathrm{~Hz}$ with a $20 \mathrm{~cm}$ aperture receiver to perform the ranging. The instrument also uses a digital approach for range detection and pulse analysis. The ranging accuracy is expected to be better than 2 metres and $\sim 20 \mathrm{~cm}$ in optimum conditions. With the given, only slightly elliptical, orbit, BELA should return a consistent data set for the most if not all of the planet. The instrument is required to function in an extreme environment with the thermal issues being particularly demanding. Novel solutions have been taken to resolve these issues. BELA is described in detail and its predicted performance outlined on the basis of pre-flight testing.
\end{abstract}

The BepiColombo mission to Mercury

Edited by Johannes Benkhoff, Go Murakami and Ayako Matsuoka

$凶$ N. Thomas

nicolas.thomas@space.unibe.ch

1 Physikalisches Institut, Sidlerstr. 5, University of Bern, 3012 Bern, Switzerland

2 Deutsches Zentrum für Luft- und Raumfahrt (DLR), Institut für Planetenforschung, Rutherfordstraße 2, 12489 Berlin, Germany

3 International Space Sciences Institute, Hallerstrasse 6, 3012 Bern, Switzerland

4 Instituto de Astrofisica de Andalucia, C/ Camino Bajo de Huétor 24, 18008 Granada, Spain

5 Max Planck Institute for Solar System Research, Justus-von-Liebig-Weg 3, 37077 Göttingen, Germany

6 Department of Geophysics, Stanford University, 397 Panama Mall, Stanford, CA 94305, USA

7 Thales-Alenia Space Schweiz (formerly at RUAG Space), Schaffhauserstrasse 580, 8052 Zürich, Switzerland

8 Hensoldt Optronics GmbH (formerly Cassidian Optronics GmbH), Carl-Zeiss-Strasse 22, 73447 Oberkochen, Germany 
Keywords Mercury · BepiColombo · Instrument · Geophysics · Topography · Interior

Acronyms

\begin{tabular}{|c|c|}
\hline $\mathrm{ADC}$ & Analogue to Digital Converters \\
\hline ADR & Altimetry Data Record \\
\hline AEU & Analogue Electronics Unit \\
\hline APD & Avalanche Photodiode \\
\hline APD-A & Avalanche Photo Diode-Assembly (board) \\
\hline APSO & Adaptive Particle Swarm Optimization \\
\hline AWG & Arbitrary Waveform Generator \\
\hline BELA & BepiColombo Laser Altimeter \\
\hline BEO & Back-End Optics \\
\hline BEX & Beam Expander \\
\hline $\mathrm{BFO}$ & Basis Function filter Output \\
\hline BPU & Baseplate Unit \\
\hline CAD & Computer-Aided Design \\
\hline CAM & Computer-Aided Manufacturing \\
\hline $\mathrm{CCA}$ & Circuit Card Assembly \\
\hline CFRP & Carbon-Fibre Reinforced Polymer \\
\hline $\mathrm{COO}$ & Collimating Objective of the BEO \\
\hline $\mathrm{CP}$ & Control Packet \\
\hline CTE & Coefficient of Thermal Expansion \\
\hline CW & Correlation Window \\
\hline CWS & Correlation Window Samples \\
\hline DC & Direct Current \\
\hline DLR & Deutsches Zentrum für Luft- und Raumfahrt \\
\hline DP & Data Packet \\
\hline DPM & Digital Processing Module \\
\hline ELU & Electronics Unit \\
\hline EMI & Electro-Magnetic Interference (filter) \\
\hline EQM & Engineering and Qualification Model \\
\hline ESA & European Space Agency \\
\hline ESTEC & European Space Research and Technology Centre \\
\hline FM & Flight Model \\
\hline FOV & Field of View \\
\hline FPA & Focal Plane Assembly \\
\hline FPGA & Field Programmable Gate Array \\
\hline GALA & Ganymede Laser Altimeter \\
\hline GLAS & Geoscience Laser Altimeter System \\
\hline HV & High Voltage \\
\hline IAA & Instituto de Astrofisica de Andalucia \\
\hline IMO & Imaging Objective of the BEO \\
\hline IR & Infrared Radiation \\
\hline JAXA & Japan Aerospace Exploration Agency \\
\hline JUICE & JUpiter ICy moons Explorer \\
\hline LCM & Laser Control Module \\
\hline LDA & Laser Diode Assembly \\
\hline
\end{tabular}




\begin{tabular}{|c|c|}
\hline LDD & Laser Diode Driver \\
\hline LEU & Laser Electronics Unit \\
\hline LHB & Laser Head Box \\
\hline LIDAR & Light Detection and Ranging \\
\hline LOLA & Lunar Orbiter Laser Altimeter \\
\hline LVDS & Low Voltage Differential Signaling \\
\hline LVIS & Laser Vegetation Imaging Sensor \\
\hline M1, M2 & Primary and Secondary Mirrors of the RTL \\
\hline M-EQM & Mechanical Engineering and Qualification Model \\
\hline MESSENGER & Mercury Surface, Space Environment, Geochemistry and Ranging \\
\hline MLA & Mercury Laser Altimeter \\
\hline MLI & Multilayer Insulation \\
\hline MMO & Mercury Magnetospheric Orbiter \\
\hline MOLA & Mars Orbiter Laser Altimeter \\
\hline MPO & Mercury Planetary Orbiter \\
\hline MPS & Max-Planck-Institut für Sonnensystemforschung \\
\hline N/A & Not Applicable \\
\hline NASA & National Aeronautics and Space Administration, \\
\hline Nd:YAG & Neodymium-Doped Yttrium Aluminum Garnet \\
\hline NLRI & NEAR Laser Ranging Investigation \\
\hline OPM & Off-axis Parabolic Mirror \\
\hline PBS & Primary Boot Software \\
\hline PCB & Printed Circuit Board \\
\hline PCM & Power Converter Module \\
\hline PDU & Power Distribution Unit \\
\hline PFD & Probability of False Detection \\
\hline PPS & Pulse Per Second \\
\hline RBU & Receiver Baffle Unit \\
\hline RFM & Rangefinder Module \\
\hline RMS & Root Mean Square \\
\hline RTL & Receiver Telescope \\
\hline RTP & Rubidium Titanyle Phosphate \\
\hline RW & Range Window \\
\hline RWS & Range Window Samples \\
\hline $\mathrm{Rx}$ & Receiver \\
\hline $\mathrm{S} / \mathrm{C}$ & Spacecraft \\
\hline SCD & Semiconductor Devices \\
\hline SLA & Shuttle Laser Altimeter \\
\hline SM & Secondary Mirror \\
\hline SOB & Spacecraft Optical Bench \\
\hline SPDT & Single Point Diamond Turning \\
\hline SPU & Straylight \& Contamination Protection Unit \\
\hline SpW & Spacewire \\
\hline SSP & Simple Serial Protocol \\
\hline STM & Structural Thermal Model \\
\hline TBU & Transmitter Baffle Unit \\
\hline TC/TM & Telecommand/Telemetry \\
\hline TEC & Thermoelectric Cooler \\
\hline TIA & Transimpedence Amplifier \\
\hline
\end{tabular}




$\begin{array}{ll}\text { Tx } & \text { Transmitter } \\ \text { UBE } & \text { University of Bern } \\ \text { UHMW-PE } & \text { Ultra-high-molecular-weight Polyethylene } \\ \text { UV } & \text { Ultraviolet } \\ \text { VGA } & \text { Variable Gain Amplifier } \\ \text { VUV } & \text { Vacuum Ultraviolet } \\ \text { WFE } & \text { Wavefront Error }\end{array}$

\section{Introduction}

In 2004 it was decided to design a laser altimeter for the European Space Agency's BepiColombo mission to Mercury. This instrument became the BepiColombo Laser Altimeter (BELA), - the first long range laser altimeter for interplanetary flight to be developed in Europe - and was selected for flight in 2005.

Laser altimetry is a powerful remote sensing technique although the measurement concept is simple (Hofton et al. 2000). The time-of-flight of a pulse of light from a laser to a reflecting surface and back is measured. The range, $z$, from the laser to the illuminated spot on a surface is related to the laser pulse time of flight by

$$
z=\frac{c \Delta t}{2}
$$

where $\mathrm{c}=299792458 \mathrm{~m} / \mathrm{s}$ (Cohen and Taylor 1997). Hence, a $1 \mathrm{~ns}$ delay corresponds to approximately $15 \mathrm{~cm}$ range. Ancillary information such as laser location and pointing at the time of each laser shot, enables the laser footprint to be geolocated in a global reference frame. It is this position in 3D space and the accuracy of the measurement that makes laser altimetry such a powerful technique for in-orbit studies of planetary bodies. While numerous short-range devices have flown on spacecraft missions to small bodies (e.g. the NEAR Laser Ranging Investigation (NLRI) Cole et al. 1997, the OSIRIS-REx Laser Altimeter (OLA), Daly et al. 2017, and the Hayabusa 2 LIDAR Watanabe et al. 2017), we are here concerned with medium to long range ( $>200 \mathrm{~km}$ ) altimetry from orbit around a planet.

Early medium-range laser altimeter systems were flown onboard the Apollo 15, 16, and 17 missions. More recently, laser altimeters flown in space include the Shuttle Laser Altimeter (SLA) (Bufton et al. 1995), the Clementine laser altimeter (Smith et al. 1997), the Mars Orbiter Laser Altimeter (MOLA) (Zuber et al. 1992), the Geoscience Laser Altimeter System (GLAS) (Abshire et al. 1998), the lunar Laser Altimeter (LALT) on the Kaguya/Selene mission (Araki et al. 2009), LAM and LLRI on Chang'E-1 and Chang'e-2 and Chandrayaan1, respectively (Jin et al. 2013), and the Lunar Orbiter Laser Altimeter (LOLA) (Smith et al. 2010). Results from the latter have recently been summarized by Smith et al. (2017) showing the power of this technique. These missions demonstrated that metre-level topography of the Earth and other planets is routinely obtainable using this technique. Recent advances in ranging and processing techniques and the digital recording of the return laser pulse shape in airborne systems such as the laser vegetation imaging sensor (LVIS) show that decimetrelevel of accuracy is now obtainable from medium-large footprint systems (Blair et al. 1999).

NASA's MESSENGER mission has provided the most detailed observations of Mercury to date. The MESSENGER spacecraft carried a laser altimeter, MLA (Mercury Laser Altimeter) that was described by Cavanaugh et al. (2007). An example of the results derived from the data can be found in Zuber et al. (2012). A more detailed discussion is presented in the next section as it provides an important benchmark for the BELA instrument. 
Table 1 Comparison of the nominal orbits of MESSENGER (Solomon et al. 2001; Mazarico et al. 2014) and the MPO (project start). The table illustrates the advantage of MPO for laser altimetry

\begin{tabular}{|c|c|c|c|}
\hline & \multicolumn{2}{|c|}{ MESSENGER } & \multirow[t]{2}{*}{ BepiColombo/MPO (original design) } \\
\hline & Initial & After 1 year & \\
\hline Periherm $[\mathrm{km}]$ & $200 \mathrm{~km}$ & & 400 km (now 480 km) \\
\hline Apoherm $[\mathrm{km}]$ & $15000 \mathrm{~km}$ & $12570 \mathrm{~km}$ & $1500 \mathrm{~km}$ \\
\hline Period $[\mathrm{h}]$ & $12 \mathrm{~h}$ & $8 \mathrm{~h}$ & $2.32 \mathrm{~h}$ \\
\hline Eccentricity & 0.74 & 0.7 & 0.162 (now 0.149 ) \\
\hline Periherm latitude & $\sim 60^{\circ} \mathrm{N}$ & & $20^{\circ} \mathrm{N}$ (now $16^{\circ} \mathrm{N}$ ) with slow rotation south \\
\hline
\end{tabular}

The BepiColombo mission is described in detail in Benkhoff et al. (2021) and we only briefly summarize key aspects here. BepiColombo comprises two main spacecraft, the Mercury Planetary Orbiter (MPO) and the Mercury Magnetospheric Orbiter (MMO), which are brought into orbit around the planet using a transfer module. The MMO is provided by the Japan Aerospace Exploration Agency (JAXA) and will enter a fairly elliptical orbit (nominally $400 \mathrm{~km} \times 19,200 \mathrm{~km}$ ) that is optimum for magnetospheric studies but less well suited to mapping of the planet's surface. The MPO is provided by the European Space Agency (ESA) and will enter a fairly circular intermediate orbit. During the design phase, the nominal orbital axes were $400 \mathrm{~km} \times 1,500 \mathrm{~km}$ above the surface. This was modified during the latter stages of the instrument development (Table 1). The near circular orbit is close to ideal for surface mapping. The current mission design will place MPO in an orbit with the periapsis slightly north of the equator. The periapsis will rotate southward with time (Benkhoff et al. 2021). The prime mission is intended to last one Earth year.

The science requirements for the MPO included goals for a laser altimeter as part of a geophysics package for studying the topography and internal structure of Mercury. This package also includes radio science (Iess et al. 2021), accelerometers (Iafolla et al. 2021), imaging (Cremonese et al. 2020) and other payload elements. The payload definition document (SCI-A/2002/007/Dc/CE, iss. 3, rev. 0; 23 May 2003) noted that no space heritage for space-borne laser altimetry was available in Europe at the time. Hence, the subsequent selection and development of a laser altimeter for MPO was a significant first for ESA. BELA, while experiencing several difficulties in development, has subsequently become a pathfinder for other European instruments such as the Ganymede Laser Altimeter (GALA) that is slated to fly on ESA's JUpiter ICy moons Explorer (JUICE) mission in 2023 (Hussmann et al. 2019).

In the following sections, we shall describe the science objectives and the instrument concept for BELA. We will then describe the sub-systems individually followed by a brief summary of the system level tests and expected science performance based upon the test results. We finish with some simple conclusions.

\section{Scientific Objectives}

The scientific objectives of BELA are to measure

- the figure parameters of Mercury to establish accurate reference surfaces

- the topographic variations relative to the reference figures and a geodetic network based on accurately measured positions of prominent topographic features 
- the radial tidal deformations of the surface

- to measure the rotation state of Mercury, including obliquity and libration amplitude

- the surface roughness, local slopes and albedo variations globally and within permanently shaded craters near the poles

BELA forms an integral part of a larger geodesy and geophysics package, incorporating radio science and stereo imaging. Although the package consists of stand-alone instruments, only the synergy between these will provide full scientific return. The synergy will support determination of planetary figure and gravity field determination, interior structure exploration, surface morphology and geology, and extend into the measurement of tidal deformation (see Genova et al. 2020 for an overview of the geodesy and geophysics investigations of BepiColombo). The offset between the centre of mass and the centre of figure will also be derived. The reference surfaces and the geodetic network will provide the coordinate system for any detailed geological, physical, and chemical exploration of the surface (see Rothery et al. 2020 for an overview of the surface and composition investigation of BepiColombo). The topography is needed to develop digital terrain models which will allow quantitative study of the geology, tectonics, and age of the surface. As an example, the detailed characterization of the $\mathrm{km}$ deep but only a few $10 \mathrm{~s}$ of $\mathrm{km}$ wide pits on Mercury will provide insights into their creation mechanism and through this to the thermal history of Mercury. BELA will contribute to BepiColombo's surface and composition investigation by providing topographic data, surface roughness at various scales and albedo measurements at the laser wavelength of $1064 \mathrm{~nm}$ (see also Rothery et al. 2020). These measurements will also be essential for investigating volatile deposits in the permanently shadowed areas in the polar regions of both hemispheres. The topography is further needed for a reduction of the gravity field data because topographic contributions to gravity must first be removed before using gravity anomalies for the investigation of sub-surface structures. The use of topography together with gravity data will constrain the characteristics of the lithosphere by an admittance analysis and a flexure model. Examples include the lithosphere elastic thickness (essential for the reconstruction of the thermal history of Mercury) and the crustal density (essential for the construction of a Hermean interior structure model). The moment of inertia will be obtained from accurate measurements of the libration amplitude and obliquity of Mercury. The moments of inertia and the tidal deformations, measured by BELA and the radio science instrument, will place further constraints on global models of the interior structure, including e.g., the size of a solid inner core (Steinbrügge et al. 2018). We note that Mazarico et al. (2014) and more recently Thor et al. (2021) detected the lunar body tide using LOLA data from onboard the Lunar Reconnaissance Orbiter. BELA will contribute to these objectives by determining the deformation of the Hermean surface while the radio science package will measure the mass relocations. Under favourable conditions, it will even be possible to constrain the rheology of the interior of the planet by measuring the time lag between the motion of the tidal bulge and the disturbing potential.

MLA was a very powerful instrument and comparable in capability, under similar conditions, to BELA (Zuber et al. 2012). However, the eccentric orbit of the MESSENGER spacecraft (Table 1) resulted in good quality data being acquired only in the northern hemisphere. The southern hemisphere of Mercury is almost completely unexplored with laser altimetry (Perry et al. 2015). The rotation of the orbit of MPO, bringing the periapsis to southern latitudes, is therefore of significance and implies that BELA is likely to make a major contribution to Mercury studies beyond that made by MLA.

The main observations to be performed by BELA are itemised in Table 2. The basic instrument concept and the link budget were developed to perform these observations and respond to the science goals from the nominal orbit. 
Table 2 Main observation types of BELA mapped to the science goals

\begin{tabular}{|c|c|}
\hline $\begin{array}{l}\text { Observation } \\
\text { type }\end{array}$ & Goal \\
\hline 1 & $\begin{array}{l}\text { Obtain a dense global grid of range measurements between S/C and Mercury's surface } \\
\text { throughout the whole science mission phase }\end{array}$ \\
\hline 2 & Measure the topography of selected morphologic features \\
\hline 3 & $\begin{array}{l}\text { Measure periodic surface deformation as a function of time (i.e. as a function of true anomaly } \\
\text { of Mercury in its orbit around the Sun) }\end{array}$ \\
\hline 4 & Assist in determining Mercury's pole position and rotation rate \\
\hline 5 & Assist in determining periodic variations of Mercury's rotation rate (physical librations) \\
\hline 6 & $\begin{array}{l}\text { Measure the albedo globally (at laser wavelength of } 1064 \mathrm{~nm} \text { ) actively (with laser pulse) } \\
\text { including in permanently shadowed regions in craters near the poles. }\end{array}$ \\
\hline 7 & $\begin{array}{l}\text { Measure the albedo (at Laser wavelength of } 1064 \mathrm{~nm} \text { ) passively (without Laser pulse) on the } \\
\text { Sun-illuminated surface }\end{array}$ \\
\hline 8 & Measure surface roughness globally and locally by analysing the shape of the return pulse \\
\hline
\end{tabular}

\section{Basic Instrument Concept and Link Budget}

\subsection{Direct Detection v. Photon-Counting}

Although there are significant differences in the exact implementations of laser altimeter systems used in interplanetary space, all of them to date have used the "classical" (direct detection) approach to laser altimetry which uses a high power laser (typically 20-50 mJ) at a low pulse repetition frequency (typically 1-10 Hz). An alternative approach is to use a high repetition rate (typically $1 \mathrm{kHz}$ ), low laser power, photon-counting approach (e.g. Blazej et al. 2007). This was studied in an early phase but it was recognized that the development time and costs for technologies not previously flown at all were likely to be prohibitive. Hence, the classical, high power laser approach was adopted.

With the choice of the classical approach, we can easily define three measurable quantities (Gardner 1992). The total number of photons, $N$, in the return pulse is related to the albedo of the surface and can be measured using

$$
N=\int_{0}^{\infty} p(t) d t
$$

where $p(t)$ is the photon flux, i.e. the number of photons received in a short interval of time. The time of flight measurement, which is our primary measurement and related to the surface elevation, is then illustrated through the equation

$$
T_{p}=\frac{1}{N} \int_{0}^{\infty} t p(t) d t
$$

and finally, the width of the return pulse, which is related to surface roughness, is given by

$$
\sigma_{P}=\frac{1}{N} \int_{0}^{\infty}\left(t-T_{P}\right)^{2} p(t) d t
$$

The BELA instrument has been designed to place constraints on all of these three parameters $\left(N, T_{p}, \sigma_{P}\right)$ for every pulse returned from the surface. 


\subsection{Digital Signal Processing}

For direct detection laser altimeters, photon return rates of $10^{2}-10^{4}$ photons per pulse (Gardner 1992; Zuber et al. 1992) are typical. Most laser altimeters developed in the past (e.g. the NEAR Laser Ranging Investigation, NLRI) detected the return pulse using analogue electronics with thresholding algorithms (e.g. leading-edge detection). This form of detection is quite robust but has the disadvantage that there is "range walk" - fixed threshold, leadingedge detection, produces an earlier trigger if the signal is higher. Multiple thresholds can be used to address this at the cost of added complexity.

The GLAS instrument (Abshire et al. 2005) used a hybrid technique where analogue leading edge detection was coupled with a subsequent digitization of the waveform. The digitized signal was then downlinked for Earth-based processing. GLAS was in Earth-orbit and a large data volume could be downlinked. This approach could not be used unmodified at Mercury by BELA because of the need to keep the telemetry rate in the $1-3 \mathrm{kbit} / \mathrm{s}$ range. The approach adopted in the BELA instrument was therefore to digitize the signal immediately and fit the return pulse in firmware, transmitting only the parameters of the fit. This has the advantage of finding the peak exactly but also allows the digitized signal to be downlinked intermittently for on-ground verification purposes and more detailed study. It does have the disadvantage that the range accuracy is dependent upon the digitization rate. At the time of the design of BELA, $40 \mathrm{MHz}$ analogue to digital converters (ADCs) were available in space-qualified quality but this was not considered to be fast enough. Hence, 2 phase-shifted ADCs were combined to provide $80 \mathrm{MHz}$ sampling. With improvements in technology, future instruments should be able to improve on these speeds but the ADCs were fast enough to allow BELA to meet its system level requirements. The Ganymede Laser Altimeter (GALA), selected for flight on ESA's JUICE mission to Ganymede, uses $200 \mathrm{MHz}$ sampling (Hussmann et al. 2019).

\subsection{Link Budget}

A simple link budget calculation is informative in revealing the main drivers for the instrument.

The distance over which a laser altimeter can range to a defined target is controlled by the product of the emitted laser pulse energy, $E_{\mathrm{t}}$, and the receiver aperture area, $A_{\mathrm{r}}$. The return pulse energy at nadir can be computed using the link equation

$$
E_{r}=E_{t} T_{r} \frac{A_{r}}{z^{2}} \frac{A_{N}}{\pi}
$$

where $E_{\mathrm{r}}$ is the received pulse energy, $T_{\mathrm{r}}$ is the receiver optics transmission efficiency, $A_{\mathrm{N}}$ is the target surface reflectivity in normal geometry, and $z$ is the altitude of the spacecraft above the surface (Zuber et al. 1992; Abshire et al. 2000). The quantity $A_{\mathrm{N}} / \pi$ is sometimes known as the target backscattering (coefficient) and has units of [ $\left.\mathrm{sr}^{-1}\right]$ (Bufton 1989). $A_{\mathrm{N}}$ is related to the geometric albedo but not in a straightforward manner because of the influence of the opposition effect - the strong increase in reflectance as one reduces the phase angle to zero. During the design phase, a nominal value of 0.26 (cf. the geometric albedo of 0.14 determined by (Robinson and Lucey 1997)) was used but a wide range of values was included in the simulations.

$T_{\mathrm{r}}$ is determined by the reflectivities of the telescope primary and secondary mirrors, the transmission of any re-focussing optics, and the transmission of a narrow-band interference filter which is used to isolate the return pulse from other light sources such as straylight and 
reflected sunlight from the planetary surface. Values of the order of 0.6-0.7 would be typical and were achieved in the BELA case (see below).

The figure of merit used to quantify the performance of a configuration is the probability of false detection (PFD). Although there are several possible ways to define this quantity, we choose it here to represent the probability that the system will fail to detect the return pulse. It should be clear from equation (2) that, assuming the instrument has the highest transmission achievable, the product of $\mathrm{E}_{t}$ and $\mathrm{A}_{\mathrm{r}}$ is the instrumental quantity that controls the performance of the system.

Equation (2) provides a first estimate of the product $E_{\mathrm{t}} A_{\mathrm{r}}$. Slightly more sophisticated results can be obtained by including other effects, namely the laser divergence, the solar background, and the distribution and magnitude of the surface slope variations. $E_{\mathrm{t}} A_{\mathrm{r}}$ was chosen to be $1.57 \times 10^{-3} \mathrm{~J} \mathrm{~m}^{2}$ offering acceptable solutions for justifiable values of the other parameters. Two key points were critical in this discussion. Firstly, the receiver telescope is effectively a large hole in the thermal protection system of the spacecraft. This allows a substantial amount of heat into the spacecraft that can significantly influence the spacecraft thermal control sub-system. Hence, the size of this hole and consequently the telescope aperture needed to be minimized. Secondly, the most efficient laser for this application is a Nd:YAG laser. A trade-off was carried out as to whether operation at $1064 \mathrm{~nm}$ or the frequency-doubled wavelength at $532 \mathrm{~nm}$ would be more power efficient. The strong reddening of the surface of Mercury, combined with the high performance of the Excelitas infrared enhanced silicon avalanche photodiode (APD), played a decisive role in this trade-off showing that greater signal to noise could be achieved at $1064 \mathrm{~nm}$.

While high laser pulse energies can be chosen, the power needed to drive this type of laser is typically factors of 20-40 times the emitted laser power. Consequently, there are natural limits to the laser pulse energy arising from the power available to the instrument and the maximum dissipation that can be allowed by the spacecraft thermal control subsystem. These factors led to a $20 \mathrm{~cm}$ diameter telescope and a $50 \mathrm{~mJ}$ pulse energy laser being baselined with a repetition frequency of $10 \mathrm{~Hz}$.

The top level characteristics of the instrument are indicated in Table 3.

\subsection{Reducing the Probability of False Detection (PFD)}

With only $\sim 1000$ photons in the return pulse, a highly sensitive detector is required. The detector of choice is a near-infrared enhanced, avalanche photo-diode (APD) (Laforce 2009). The signal from the APD must be amplified. It has been recognized that the noise floor of the APD-amplifier combination has a significant effect on the PFD when close to the detection threshold of the system. Improvements in the PFD can be made by using previously obtained good returns to predict the time of future returns (range tracking). Most altimeters activate a search for the return pulse within a range window. This window is usually set to a large value (e.g. equivalent to $30 \mathrm{~km}$ in altitude) to be sure that the return pulse is within it. However, if the pulse is found, and if the variation in altitude between each measurement is small, then the length of the range window can be reduced. This reduces the probability of the rangefinding algorithm misinterpreting a noise spike as a valid return and therefore decreases the PFD. Onboard filtering of the times of received returns can also be used in a statistical way to reduce the PFD. This led to baselining of range tracking and algorithms for the active reduction of the range window size within a software range tracking algorithm based on Bayesian techniques that was implemented within the onboard computer. 
Table 3 Top level properties of the BELA instrument

\begin{tabular}{ll}
\hline Property & Description \\
\hline Receiver aperture (nominal; effective) & $202 \mathrm{~mm} ; 198.5 \mathrm{~mm}$ \\
Laser wavelength & $1064 \mathrm{~nm}(\mathrm{Nd}:$ YAG) \\
Pulse repetition frequency & $1-10 \mathrm{~Hz}$ (nominal 10 Hz) \\
Laser pulse energy & $50 \mathrm{~mJ}$ \\
Laser beam divergence & $60 \mu \mathrm{rad}$ (full-cone) \\
Laser pulse width & $5 \mathrm{~ns}$ \\
Digitization frequency & $80 \mathrm{MHz}$ (equivalent to $1.87 \mathrm{~m})$ \\
Internal filter bandwidth & $20 \mathrm{MHz}$ (equivalent to $7.5 \mathrm{~m})$ \\
Maximum range window size & $30 \mathrm{~km}$ \\
Filter bandwidth & $1.4 \mathrm{~nm}$ \\
Receiver efficiency (telescope and filter) & 0.84 ( \pm 0.06$)$ \\
(10\% reduction expected at end-of life) & \\
Receiver detector & Excelitas custom-APD (812 $\mu \mathrm{m}$ diameter circular sensitive \\
& area) \\
Nominal quantum efficiency at 1064 nm & 0.34 \\
Mass (inc. thermal hardware) & $15.006 \mathrm{~kg}$ \\
Power (max average@ 10 Hz, standby) & $43.24 \mathrm{~W} ; 13.47 \mathrm{~W}$ \\
Data rate (min operational; max operational) & $3.17 \mathrm{kbit} / \mathrm{s} ; 9.89 \mathrm{kbit} / \mathrm{s}$ \\
\hline
\end{tabular}

\subsection{Accuracy of the Time of Flight Measurement}

There were two other important elements in the design that are needed to ensure accurate measurement of the time of flight. Firstly, the BELA instrument clock is based around an oscillator that is required to be stable. Rather than ensuring stability against drift over many years, it is more efficient and reliable to calibrate the internal timing against a reference. It was decided that this should be performed by using a pulse per second (PPS) from the spacecraft which could itself be calibrated through spacecraft telemetry. BELA was therefore required to count the number of internal clock cycles between each PPS from the spacecraft to calibrate internal drift. All times of flight are referenced to the number of clock cycles per second counted by the instrument. Secondly, it was necessary to guarantee that the time of the outgoing pulse could be accurately linked to the time of the return pulse. This is best achieved by using the receiver electronics to detect both the outgoing and the incoming pulse. Any drifts of the timing of the pulse emission with respect to pulse detection would then be irrelevant. It was decided to take a small fraction of the emitted pulse from the laser beam and feed it, using optical fibres, into the receiver where the detection of an emitted pulse would initiate the time of flight measurement (see Fig. 1).

\subsection{Physical Design Drivers}

There were three main challenges in generating a design to meet the requirements - thermal control, low mass, and limited power.

The thermal load on the instrument is significant. While the low orbit implies that Mercury itself shields the instrument from the Sun at phase angles greater than $142^{\circ}$, sunlight can still reach the instrument directly in the range $90^{\circ}-142^{\circ}$. In addition, reflected light and 


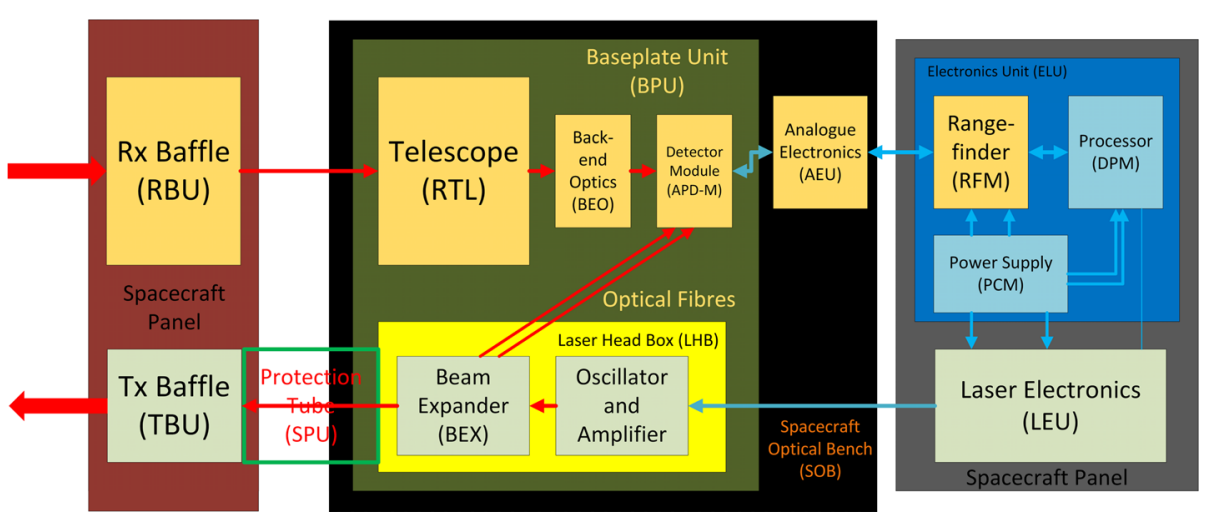

Fig. 1 Functional block diagram of the BELA instrument. The instrument building blocks are indicated with the transmitter at the bottom and the receiver at the top. The electronics are to the right. Optical signals are in red, electrical signals in light blue. Multiple lines from one box to another indicate redundancy. Orange boxes define the receiver (Rx), light green boxes define the element of the transmitter (Tx). Further details on the components are provided in Table 4

Table 4 Description of the individual units of BELA and their functions (from Thomas et al. 2019)

\begin{tabular}{lll}
\hline Unit & Acronym & Purpose \\
\hline Electronics Unit & ELU & $\begin{array}{l}\text { The ELU contains the DPM (Digital Processing Module), the PCM } \\
\text { (power converter module) and the RFM (Rangefinder module, i.e. the } \\
\text { digital part of the rangefinder electronics) in one box. This box has the } \\
\text { only direct electrical interface to the spacecraft. }\end{array}$
\end{tabular}

Laser Electronics Unit LEU The LEU controls the laser and provides the laser with power (dedicated power converter).

Transmitter Baffle Unit

The TBU has no mechanical or thermal (conductive) connection to any other part of BELA, but is mounted directly to a dedicated support bracket, provided by the spacecraft. It prevents sunlight reaching the beam expander and the laser head box.

Straylight \&

Contamination

Protection Unit

Receiver Baffle Unit

RBU

Analogue Electronics Unit

Baseplate Unit

SPU
The SPU is mounted on the spacecraft optical bench and closes the gap between the transmitter aperture (the beam expander last surface) and the TBU and provides protection against contamination and straylight.

The RBU limits the absorption of direct sunlight entering the receiver aperture. It has no mechanical or thermal (conductive) connection to any other part of BELA. It is mounted directly to a dedicated support bracket, provided by the spacecraft.

AEU An electronics box which contains a high voltage $(<500 \mathrm{~V})$ source for the APD, and proximity electronics for the rangefinder (analogue electronics).

BPU The BPU comprises

- a baseplate upon which other elements are mounted

- the receiver telescope (RTL)

- the Rx Focal Plane Assembly (FPA), which includes the Back-End Optics (BEO) (which itself includes an interference filter for isolating the $1064 \mathrm{~nm}$ laser light), the avalanche photo-diode (APD) detector

- the laser head box (LHB) incl. the beam expander required to produce the required footprint diameter. Two optical fibres take a fraction of the emitted pulse to the Rx FPA to support detection of the time of the emitted pulse. 
thermal IR emission from Mercury's sunlit hemisphere provides a further large heat load over the orbital period. Hence, the thermal design was recognized as being critical at an early stage of the design process.

Orbital laser altimeters can be developed for $<8 \mathrm{~kg}$ and $<25 \mathrm{~W}$ peak (e.g. Cavanaugh et al. 2007). The original target mass and power allocations of $12 \mathrm{~kg}$ and $40 \mathrm{~W}$ might therefore appear generous. However, the mass needed to include all electronics, ther$\mathrm{mal} /$ protection hardware and control (including Peltier elements and baffles), and harness. This led to a number of hard decisions on instrument properties. It will be seen in the instrument description how these constraints were addressed.

\subsection{Distribution of Activities}

At the time of the proposal, it was already clear that the cost of development of the instrument would exceed the resources available within any one member state of ESA. It was therefore decided that a Swiss-German consortium (with support from a Spanish institute) should divide the responsibilities roughly equally. The resulting consortium comprised the German Aerospace Centre (Institute for Planetary Research) (DLR), the Max-PlanckInstitut für Sonnensystemforschung (MPS), the Instituto de Astrofisica de Andalucia (IAA), and the University of Bern (UBE) supported by their local industries.

While other options were considered, the best solution appeared to be that DLR would take over the transmitter (Tx) section of the instrument and the control electronics with support for the laser from MPS, while UBE would build the receiver (Rx) section and perform the system integration. This approach both played to the strengths of the two parties and, more critically, allowed the Rx and Tx parts of the instrument to be tested independently before system level integration. IAA took responsibility for the power converter module which was needed very early in the project to support this concept. A functional block diagram is shown in Fig. 1 indicating the individual elements and the electrical, optical and mechanical interfaces between the elements.

The individual units of the laser altimeter and their acronyms are itemized in Table 4. To give a less abstract view of the system, Fig. 2 shows an image of the system without baffles and cables acquired prior to shipment for spacecraft integration. The units, which are marked together with some key components, are roughly in the relative positions indicated by the schematic diagram in Fig. 1. The baffles were integrated directly to the spacecraft and their positioning is shown in Fig. 3.

The next sections of the text describe the individual elements. We begin by describing the transmitter section of the instrument.

\section{Transmitter}

The development of the dedicated BELA laser system design inherited major aspects of reliable range finders at Carl Zeiss Optronics (now Hensoldt Optronics) in Oberkochen, Germany (Becht et al. 2010). The actual hardware consists of two boxes, the lightweight laser head box (LHB; Fig. 4 Kallenbach et al. 2013) isostatically mounted onto the BELA instrument baseplate (Fig. 1) and the laser electronics unit (LEU; Fig. 5) mounted onto the spacecraft bench, providing laser voltage and current supply as well as control communication for the LHB on one side and a command interface to the electronics unit of the BELA instrument on the other side. 


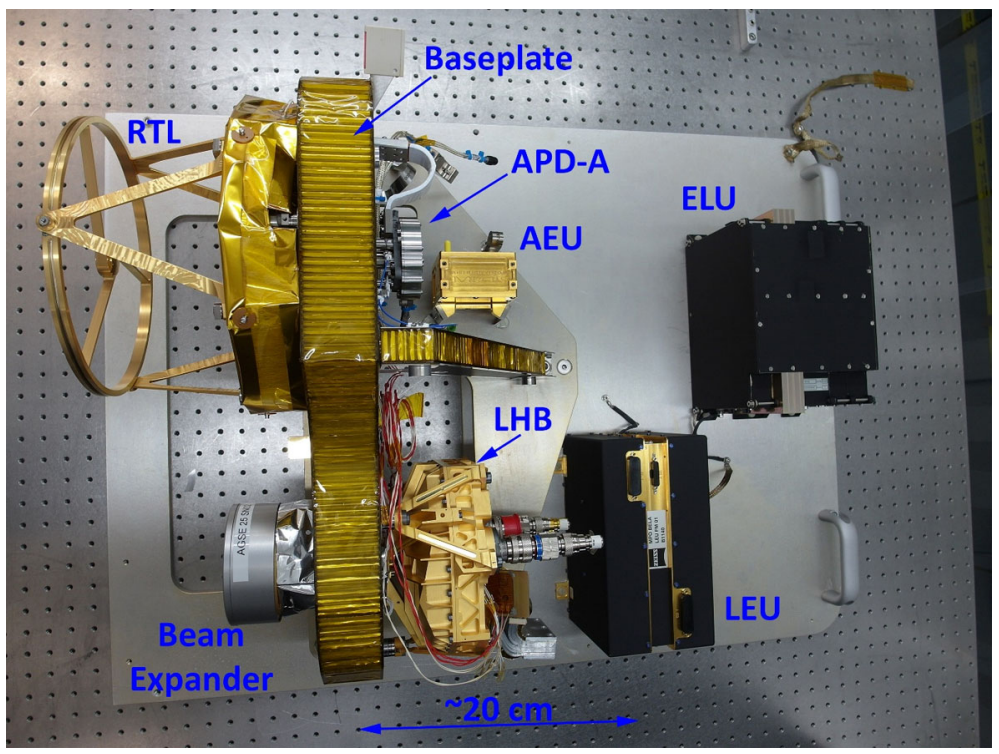

Fig. 2 The BELA system (without cables) on the bench at the time of final inspection. The baffle units and the Straylight \& Contamination Protection Unit (SPU) are not shown. The individual units and some key elements are marked. An approximate scale is given

\subsection{Optical Design}

The Laser Head Box (LHB) contains two cold-redundant Nd:YAG laser systems both emitting pulses at $1064 \mathrm{~nm}$ wavelength with typically $50 \mathrm{~mJ}$ energy, a pulse length of $5 \mathrm{~ns}$ and an adjustable pulse repetition rate of 1 to $10 \mathrm{~Hz}$. The schematic of the single-stage, actively q-switched, diode laser side-pumped Nd:YAG rod laser system with unstable resonator geometry is illustrated in Fig. 6. Only a few key features of the laser system are summarized here. Technical details and verification test results have already been reported in Kallenbach et al. (2013) and Weidlich et al. (2011).

The four pump laser diode assemblies (LDAs) of each cold-redundant Nd:YAG laser are driven in series by 100 A diode laser current pulses with a length of up to $200 \mu$ s supplied from a $300 \mathrm{~V}$ capacitor reservoir. The LDAs emit at about $808 \mathrm{~nm}$ wavelength with an electrical-to-optical efficiency of about $50 \%$. The pump light is converted with an opticalto-optical efficiency of about $10 \%$ to laser radiation by means of a pump cavity of diffusing reflectors made from purified polytetrafluorethylene material. In order to extract the stored energy at this high efficiency into a short laser pulse at $1064 \mathrm{~nm}$, an electro-optic crystal (Pockels cell) switches from non-polarizing to quarter-wavelength condition within less than $30 \mathrm{~ns}$ rise time to actively open the optical path in the laser resonator. This electro-optic switch is driven by a miniaturized $3 \mathrm{kV}$ transistor cascade in close proximity to the laser resonator inside the LHB. With this active q-switch, typical pulse durations of $5 \mathrm{~ns}$ are achieved at $1064 \mathrm{~nm}$ as required by the application.

During its few round trips, the stimulated laser beam pulse at $1064 \mathrm{~nm}$ expands in diameter. This allows for subsequent stimulation of radiation from the entire laser rod volume. An out-coupling mirror with radially variable reflectivity determines the mode profile and establishes a near Gaussian mode shape of the laser pulse leaving the resonator in direction of the beam expander (BEX) optics. The outgoing laser beam has a divergence of 40-60 $\mu$ rad after 
Fig. 3 The baffles were integrated directly onto the spacecraft. This image shows the baffles and their positioning with respect to the baseplate

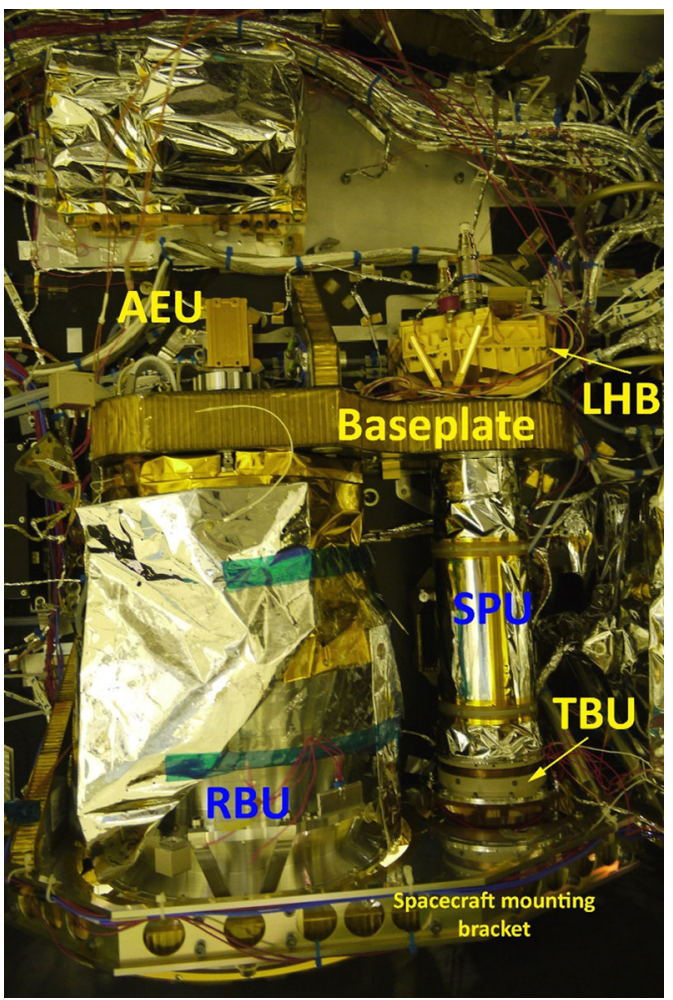

leaving the BEX and reaches Mercury's surface with spot sizes between 16 and $90 \mathrm{~m}$. An optical fiber leading to the BELA receiver is connected to the BEX, providing a reference to the outgoing laser pulse and the start signal for the altimetry measurement.

To protect the laser optics from laser-induced damage effects (Kallenbach et al. 2013; Riede et al. 2008, 2009, 2011), the LHB was initially pressurized with 1.24 bar of synthetic air and sealed by C-rings between the LHB structure (Fig. 7) and its cover. This ensures a pressure drop of no more than 100 mbar over the 12-year mission time. Additionally, a catalyst convertor inside the LHB housing reduces concentrations of contaminants near the laser optics. Figure 7 shows a CAD drawing of the BELA LHB.

\subsection{Structural and Thermal Design}

Launch vibrations, spacecraft separation shocks, and rapid changes of thermal conditions during the $2.3 \mathrm{~h}$ orbit may have serious consequences for laser alignment stability and, hence, for the co-alignment of the BELA transmitter and receiver. Therefore, the thermal interfaces have been separated from alignment-critical mechanical interfaces in order to reduce thermo-mechanical deformation.

The heat dissipation of the LHB is dominated by the $\sim 5 \mathrm{~W}$ of ohmic dissipation of the diode lasers. In order to minimize thermal gradients and consequent deformations, these are directly mounted onto the LHB structure which is made of highly thermally conductive AlBeMet alloy. From there, the heat flows to the heat-pipe thermal sink provided by the MPO spacecraft via a highly thermally conductive flexible link (Fig. 8, right). The optomechanical stability of the LHB structure itself (Fig. 8, left) is provided by quasi-isostatic 
Fig. 4 Engineering and Qualification Model (EQM) of the BELA Laser Head Box (LHB) without cover and C-ring sealing containing two cold-redundant laser systems and including the beam expander (BEX) optics (from Kallenbach et al. 2013)

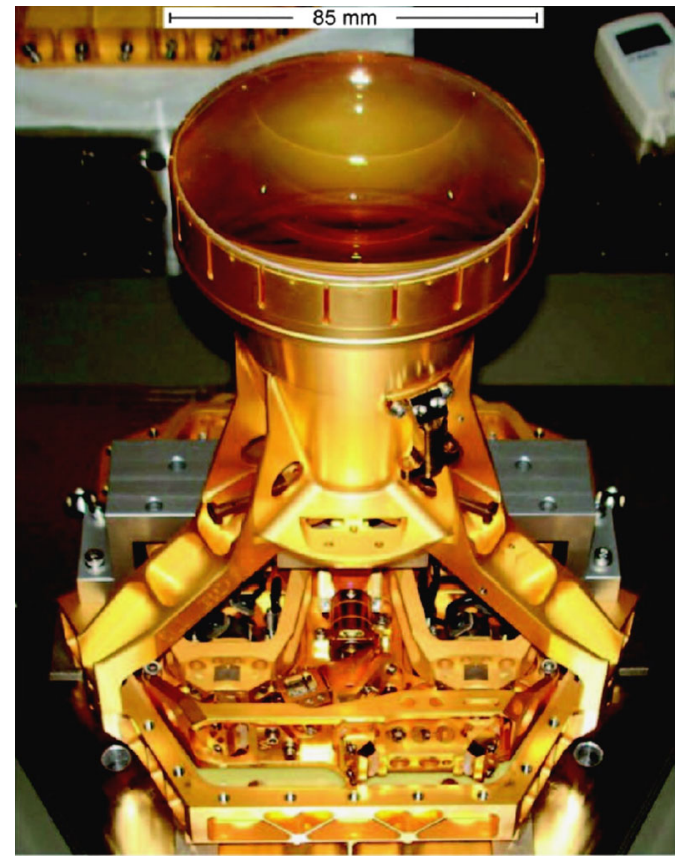

Fig. 5 Engineering and Qualification Model (EQM) Laser Electronics Unit (LEU) with capacitor reservoirs to drive the 100 A diode laser current at pulse lengths of up to $200 \mu$ s. From Kallenbach et al. (2013)

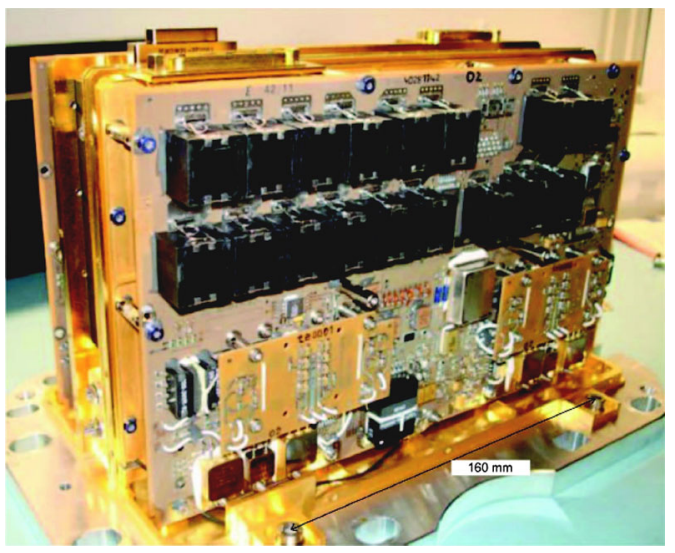

bipod flexures mounted onto the BELA instrument baseplate. These bipods have a low thermal conductivity of $0.02 \mathrm{~W} / \mathrm{K}$ and transfer only very small torques $(<0.04 \mathrm{Nm}$ over the full operational temperature range) to the instrument baseplate to avoid thermo-mechanical deformation.

The pointing of the BELA laser with respect to the receiver is determined mainly by the pointing of the BEX optics (Fig. 8, left). This resulted in a precision design with the BEX supported by quasi-isostatic mounts attached to the base unit of the LHB near its bipod fixations to the instrument baseplate. This solution separates critical optics from the deformation of the box, which is, in turn, induced by thermal expansion and pressure differences between the interior and exterior of the laser box. The BEX is additionally wrapped in multilayer insulation (MLI) in order to minimize radiative heat transfer. 
Fig. 6 Schematics of one of the BELA laser resonators

Fig. 7 BELA laser CAD drawing of the Laser Head Box (LHB) with cover removed. The second and third lens of the beam expander optics outside the pressurized volume are not shown here. From Kallenbach et al. (2013)
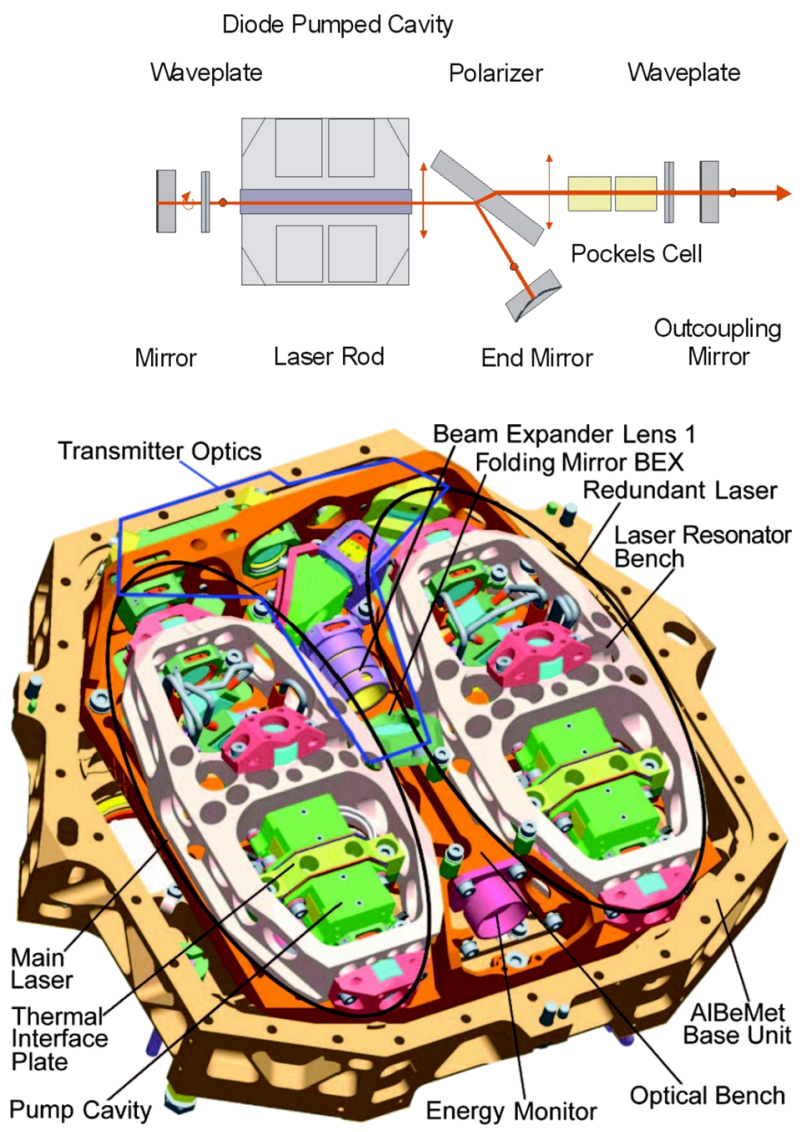

A further thermo-elastic decoupling was achieved by isostatic mounting of the optical bench onto the LHB AlBeMet structure (Fig. 7). This optical bench supports the transmitter optics and the two redundant laser resonator benches which are isostatically mounted themselves. The transmitter optics combine the two laser beams of the two cold-redundant Nd:YAG lasers to pass them through the single BEX optics. The BEX has no redundancy because of size and mass and has received special attention as a potential single point failure.

Using the AlBeMet162 alloy for the LHB structure has several advantages. The thermal expansion coefficient of AlBeMet $\left(\approx 13.9 \mathrm{ppm} \mathrm{K}^{-1}\right)$ is lower than that of aluminium and its thermal conductance is very high $\left(\approx 210 \mathrm{~W} \mathrm{~m}^{-1} \mathrm{~K}^{-1}\right)$. The density of $\approx 2.1 \mathrm{~g} \mathrm{~cm}^{-3}$ of AlBeMet and its elastic modulus of $\approx 180 \mathrm{GPa}$ is well suited to the need to keep the LHB mass within limits acceptable for the BELA instrument design and to keep the eigenfrequency of the standalone LHB system mounted on the bipods at $\geq 275 \mathrm{~Hz}$. Vibration eigenfrequencies of design elements (components and their mounting) inside the LHB have been kept at much higher frequencies than those typically arising from instrument and spacecraft excitation.

An additional heat source of the LHB/BEX system is the $1.5 \mathrm{~W}$ thermal radiation absorbed from the thermal environment outside the spacecraft and transferred from the TBU onto the BEX front lens, which is located some distance from the thermal link. Therefore, the thermo-mechanical behaviour of the LHB (including the BEX) was analyzed in detail. Based on the coefficient of thermal expansion (CTE) data for the BEX tube and the lens materials, and by including the axial temperature distribution indicated in Fig. 8, a $2 \mu \mathrm{rad}$ 

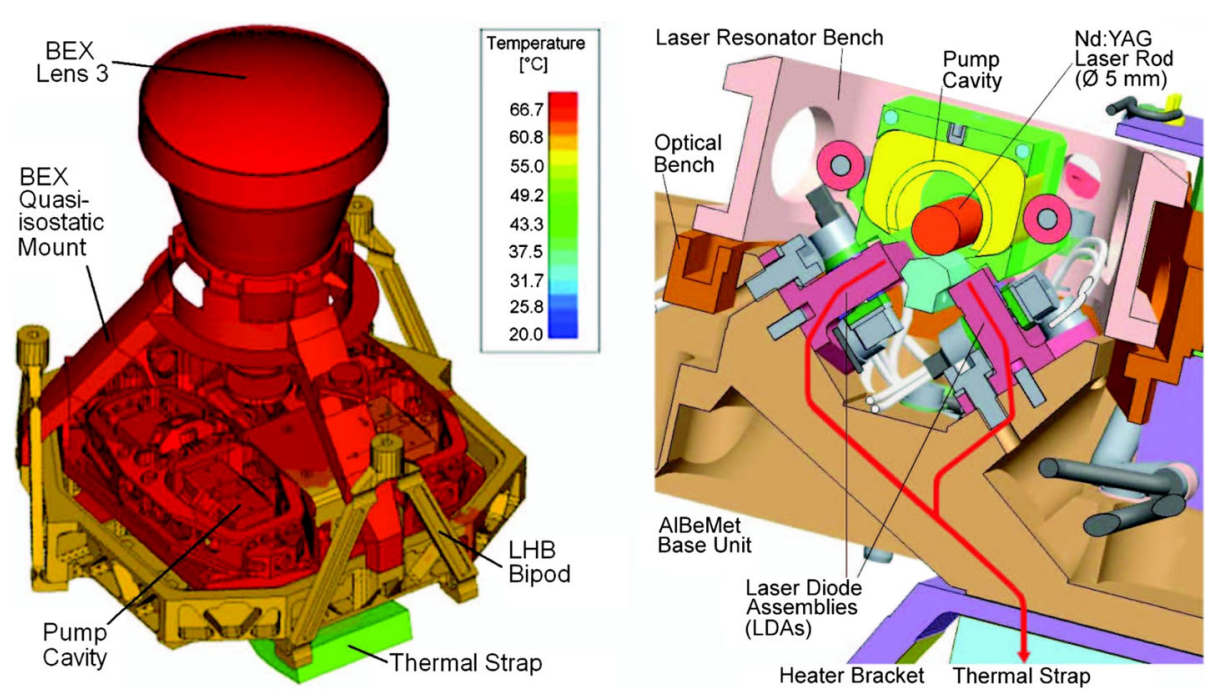

Fig. 8 Left: Laser Head Box (LHB) with removed cover and color-coded temperature distribution as derived through an ESATAN simulation software package. The calculated temperature distribution was applied to the structural mathematical model for thermos-elastic analysis. Right: CAD cross section through one of the BELA laser pump cavities indicating the heat flow from the LDAs to the thermal strap. From Kallenbach et al. (2013)

increase of beam divergence was calculated. If the temperature distribution inside the BEX front lens material and the temperature dependence of its refractive index are taken into account this effect is actually compensated. The laser pointing error caused by temperature effects does not exceed $10 \mu \mathrm{rad}$ because the laser resonator and the transmission optics reside on a structure completely made from the same AlBeMet material as described above. The transmitter's performance with respect to its thermo-optical pointing stability, alignment, pulse energy, wavelength, pulse profile and length have a direct impact on the signal quality and strength of the instrument and in consequence on the quality of science data. Therefore the transmitter was extensively tested at facilities at DLR in Berlin-Adlershof under various environmental conditions in all possible representative configurations showing that the expected performance can be fully met (Althaus et al. 2019).

\subsection{Electrical Design}

The electronics for the BELA Laser System are split between two housings connected by an external cable harness. The Laser Electronics Unit (LEU) is directly mounted onto the spacecraft panel. It comprises the majority of the electronics of the laser system and provides the electrical interface to the Electronics Unit (ELU). The ELU contains the Power Converter Module (PCM) where a first group of secondary instrument voltages, $\pm 3.3 \mathrm{~V}$, $\pm 5.2 \mathrm{~V}$, and $+12 \mathrm{~V}$, is generated from the primary $28 \mathrm{~V}$ DC voltages by fly-buck convertors and referenced to a single-point grounding in the ELU. These secondary voltages drive the small-signal circuits in both the BELA receiver and the BELA laser system. The high-power electronics of the laser system are driven by power supplies inside the LEU which are fed directly by the spacecraft primary $28 \mathrm{~V}$ line. The diode laser current pulses, with amplitude of $100 \mathrm{~A}$ and duration up to $200 \mu \mathrm{s}$, are derived from a $40 \mu \mathrm{F}$ capacitor reservoir charged 
up to $350 \mathrm{~V}$. The latter voltage source also feeds a Greinacher cascade inside the LHB to generate the $3 \mathrm{kV}$ needed to switch the Pockels' cell (active Q-switch).

\section{The LEU Comprises Three Modules}

1) The Laser Control Module (LCM) was manufactured on a single rigid-flex-rigid printed circuit board (PCB), containing main and redundant circuitry. The LCM mainly implements low-frequency control and digital interface functions.

2) The Laser Diode Driver (LDD) consists of two identical rigid PCBs, one containing main and the other redundant circuitry, implementing the $350 \mathrm{~V}$ high voltage power supply and the generation of a high current pulse to the LDA in the LHB.

3) The soft start (reduction of extreme in-rush currents into the LEU) and electro-magnetic interference (EMI) filter (LEU_EMI) is built on a single rigid-flex-rigid PCB, containing main and redundant circuitry and implements an in-rush current soft start facility and filtering of the primary $+28 \mathrm{~V}$ power.

These three modules are contained in the mechanical LEU housing containing the electronic CCAs and designed to reduce internal and external EMI.

The LHB contains the electronics to control the generation of the laser pulse and to detect its emission. The energy monitor detects the laser pulse. It is implemented on a single rigidflex-rigid PCB, containing main and redundant circuitry. The Pockels Cell Driver generates a $3 \mathrm{kV}$ pulse with less than $30 \mathrm{~ns}$ rise time to trigger the Q-switch of the laser resonator after the Nd:YAG rod has been pumped optically by the LDAs. It has been manufactured on a single rigid PCB, containing main and redundant circuitry. The LHB housing is made of AlBeMet and contains the electro-optic components and electronic CCAs. It has also designed to reduce EMI.

Simplified overall block diagrams for the LEU and LHB are shown in Fig. 9.

\subsection{Verification Testing}

Only a small selection of test results from the BELA laser Engineering and Qualification Model (EQM) and its Flight Model (FM) can be reported here. Lifetime and reliability have been verified with respect to launch vibration loads, spacecraft separation shock loads, and irradiation dose as well as thermal loads over the course of the full mission duration.

At the time of the BELA laser hardware development, qualification of laser optics and electro-optics could not be performed according to standard procedures. A special program was set-up with the help of ESA to qualify the LDAs produced by Semiconductor Devices (SCD) in Israel (type R8 in SCD nomenclature see Berk et al. 2009). A simple life test demonstrated the capability of the pump diode lasers to operate for the required 200 MShots needed for the mission.

During this life test and during other accompanying tests it turned out that the LDAs may undergo initial power degradation after a few MShots which is presumed to be caused by "infant mortality." In order to mitigate this effect, the FM LDAs underwent a burn-in of about 20 MShots to try to eradicate this risk. Special focus has also been put on critical optical components such as the RTP crystal (Rubidium Titanyle Phosphate - $\mathrm{RbTiOPO}_{4}$ ) of the Pockels' cell inside the laser resonator and the Nd:YAG laser rod. In particular, their radiation hardness has been studied (Fig. 10).

Compared to subsequent missions employing laser altimeters, e.g., the JUICE mission to the Jovian magnetosphere, a conservative approach was taken by testing against very high irradiation doses. In the representative pulsed operation mode (cf. Rose et al. 1995), 


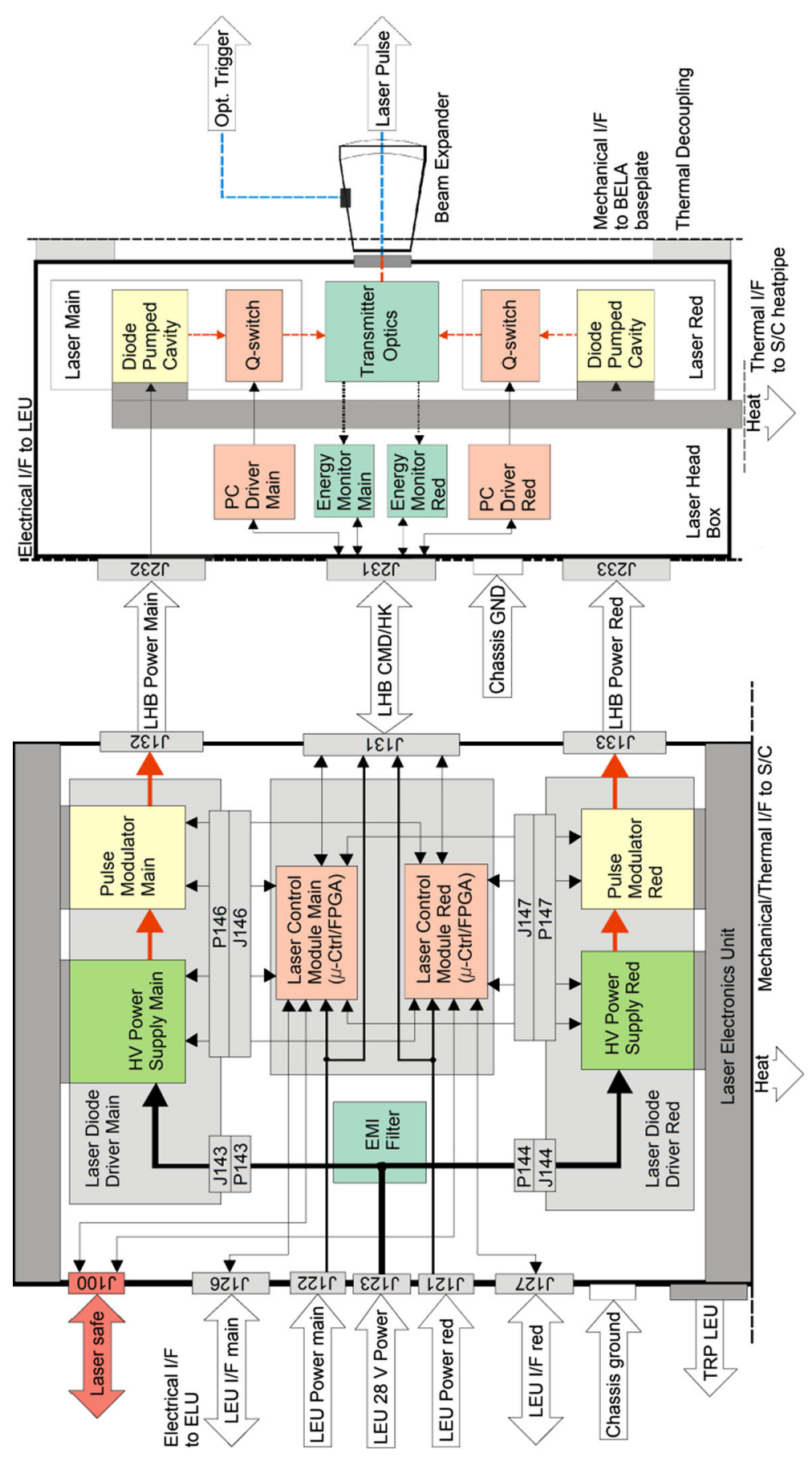

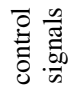

芯

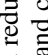

芩

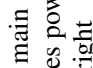

胥

넌을

这焉界

尊宁

可 吾

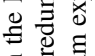

ธี ซ్ ซ్

츨

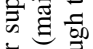

$\vec{D}$ के

․․ㄹ

志 $\frac{0}{0} \cdot \frac{0}{0}$

吾:

范

守 $\frac{0}{8}$

코 롤

怘范

约

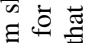

的

要

원

ช

응

过

额

謌

牙岕. 言

융

법

콭

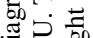

담

记

ह

造造

a

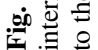



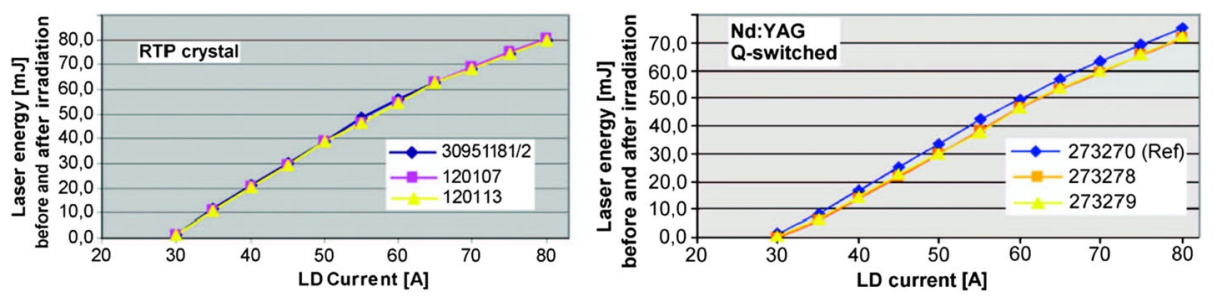

Fig. 10 Left: Laser pulse energy before irradiation of the Rubidium Titanyle Phosphate (RTP) crystal (blue dots), after $200 \mathrm{kRad}$ of proton irradiation (purple), and after additional $200 \mathrm{kRad}$ of gamma irradiation (yellow). Right: laser pulse energy before irradiation of the Nd:YAG laser rod (blue), after $200 \mathrm{kRad}$ of gamma irradiation (orange), and after additional $200 \mathrm{kRad}$ of proton irradiation (yellow)

Table 5 Boresight budget of the BELA laser system

\begin{tabular}{lc}
\hline Load contribution & $\Delta_{\mathrm{bs}}(\mu \mathrm{rad})$ \\
\hline Random vibration total & 25 \\
Shift of bipods & 23 \\
Shift of reference mirrors & 8 \\
Rotation in Earth's gravity field & 11 \\
Shock (1000 g) & 10 \\
Thermal cycling total & 44 \\
Baseplate deformation & 42 \\
Shift opto-mechanical parts & 10 \\
Total qualification loads & 54 \\
\hline
\end{tabular}

a degradation in output power of $8.3 \%$ was measured after $200 \mathrm{kRad}$ of gamma irradiation and a total degradation $11.3 \%$ was measured following an additional $200 \mathrm{kRad}$ of proton irradiation. Even this degradation in output power could be compensated by adaptation of the pump time from typically $125 \mu$ up to $200 \mu$ s. During the full mission duration of BepiColombo, the BELA Nd:YAG laser rod, however, should only experience up to 20 $\mathrm{kRad}$ of irradiation according to established models.

As part of the structural and thermal analysis and testing of the BELA instrument as a whole and of the laser system in particular, the pointing accuracy has received particular attention. Bore-sight stability remained the most critical parameter (Kallenbach et al. 2013). The boresight changes caused by random vibration testing, shock testing, reorientation of the LHB in Earth's gravity field, and during thermal cycling resulted in the boresight budget as summarized in Table 5. The loads experienced in vibration were significant. At RMS levels of $9.1 \mathrm{~g}$ at the LHB bipod footpoints at the interface to the BELA baseplate, the BEX lens experienced $40.3 \mathrm{~g}$. Despite this, the boresight remained within required limits.

The change of beam divergence during qualification testing was dominated by increases of up to $8 \mu \mathrm{rad}$ due to vibration loads and $4 \mu \mathrm{rad}$ due to thermal loads (although the measurement accuracy was estimated to be $\sim 5 \mu \mathrm{rad}$ ).

Finally, it was decided to seal the laser to limit the effects of diffusion. The leakage rate of the C-ring sealed LHB has been determined experimentally after vibration of the LHB during thermal cycling in a vacuum chamber. The synthetic air filled into the LHB has an admixture of $1 \%$ He. With the help of a helium leak detector (Alcatel ASM 180), the leakage rate was determined to vary between $2 \times 10^{-9}$ and $10^{-8} \mathrm{mbar} \mathrm{s}^{-1}$ in the temperature range between $-5{ }^{\circ} \mathrm{C}$ and $55^{\circ} \mathrm{C}$. A He leakage rate of $10^{-6}$ mbar s $^{-1}$ would correspond to a pres- 


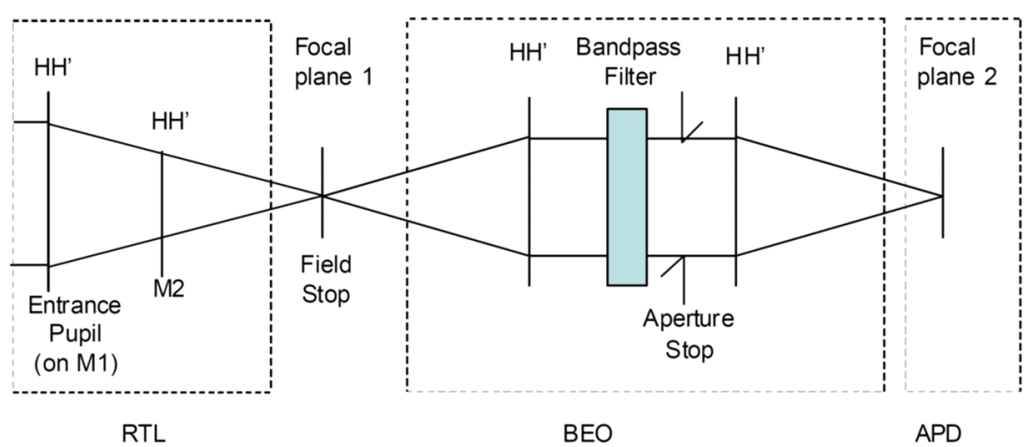

Fig. 11 The optical design concept for the BELA receiver. Light enters the receiver on the left. The back-end optics re-images the focus of the telescope and is used to house the bandpass filter before transferring the final image to the detector

sure drop from 1 bar to 900 mbar over the projected mission time. Therefore, we consider the risk as very low that the pressure inside the LHB drops below the critical value governed by the so-called Paschen curve (in our case a few millibars) at which corona discharge at the active Q-switch and its driver electronics could occur.

A summary on electromagnetic compatibility issues of the BELA transmitter and receiver can be found in Kallenbach et al. (2016) and Behnke et al. (2019).

\section{Receiver}

\subsection{Optical Design Concept}

The optical design concept for the BELA receiver is shown in Fig. 11. A Cassegrain telescope (the receiver telescope - RTL, Fig. 12) collects the photons from the return pulse. A field stop is placed at the focus of the telescope as the first step in removing straylight. The field stop is imaged by a back-end optics (BEO) that transfers the collected photons to the detector. This 1:1 imaging optic includes a narrowband filter centred at the laser wavelength to reject reflected light from the planet. It is important to note that the BEO is not symmetrical about the bandpass filter. The entrance side is longer to provide more effective straylight rejection. We now discuss the RTL in detail before describing the BEO.

\subsection{Telescope}

The original mass allocation to BELA placed very tight constraints on the receiver telescope (RTL). The design drivers were

- Low mass allowance of $600 \mathrm{~g}$ (including contingency)

- Maximum design wavefront error (WFE) of $\lambda / 10$ RMS at $1064 \mathrm{~nm}$

- Stability of WFE of $\lambda / 3$ RMS at $1064 \mathrm{~nm}$ under all operational environmental conditions

- Co-alignment of the line of sight with the optical axis within $4 \mu \mathrm{rad}$ (for alignment purposes)

- Stability of line of sight within $25 \mu \mathrm{rad}$ under all operational environmental conditions.

- High reflectivity of mirrors and inner surfaces to fulfil transmission and straylight requirements 
Fig. $12 \mathrm{CAD} / \mathrm{CAM}$ of the

BELA receiver telescope (RTL)

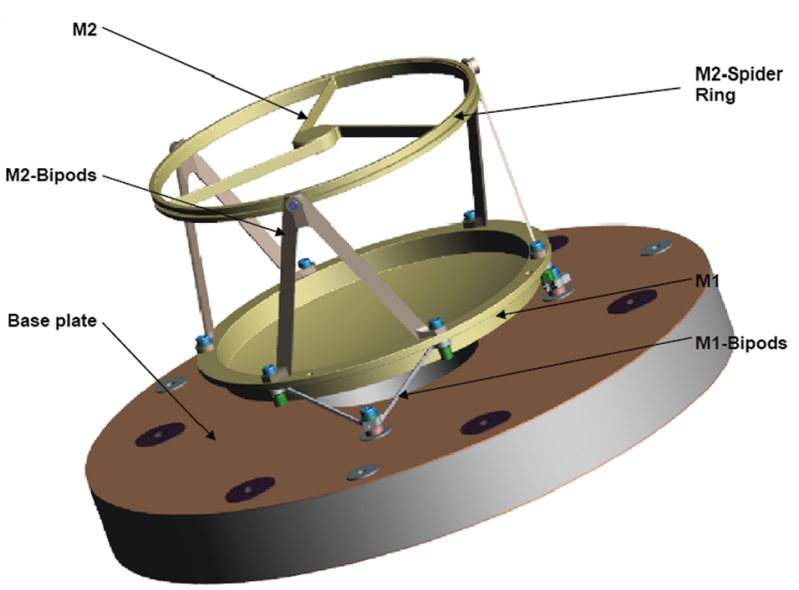

- Surface roughness of $<2.2 \mathrm{~nm}$ RMS to fulfil straylight requirements

- Extremely hot and variable thermal environment around Mercury, requiring good thermal conductivity within the telescope to smooth out temperature gradients rapidly.

Several materials were considered and rejected during a trade-off phase. Electroformed nickel was rejected because of its relatively poor thermal conductivity which would have led to distortion and loss of efficiency. Silicon carbide was considered to be too massive. An all-beryllium design was eventually chosen as a consequence of its high strength to mass ratio and good thermal properties. A compact design was chosen to reduce the overall length and therefore lower the mass. The resulting telescope met the weight allocation using a focal length of $1.25 \mathrm{~m}$, and a field of view of just over $530 \mu \mathrm{rad}$. The field of view was a trade-off between the background reflected light from the planet and the need to align and maintain the alignment of the outgoing laser pulse within the receiver field of view when the instrument was placed under thermal load.

The telescope comprises a parabolic primary mirror (M1) and a hyperbolic secondary mirror (M2). M2 was mounted on a spider-like structure also made from beryllium that was robust against thermal distortion (Fig. 13). A cross hair was drawn into the rear side of M2 within the highly reflective surface to support alignment during telescope and instrument integration (Fig. 13). The whole telescope was insulated from the baseplate using titanium washers and low conducting titanium bipods.

The manufacturing of the mirrors followed a 6 step process in order to meet the tight tolerances on the surface forms and low surface roughness:

- The raw shapes of the mirrors were manufactured from certified instrument grade beryllium (beryllium $\mathrm{I}-70 \mathrm{H}$ ) to tolerances achievable with best of class milling machines at houses qualified to process beryllium.

- The inspected and measured beryllium parts were sealed by a thin adhesion and sealing layer of chrome sputtered directly onto the beryllium. On top of this chrome layer, a gold layer was applied to further protect the chrome-coated beryllium part during the subsequent galvanic processes.

- The sealed raw mirror was plated with a sufficiently thick copper layer by applying a galvanic process.

- The shape of the mirror and the required low surface roughness of $<2.2 \mathrm{~nm}$ RMS was established by applying a Single Point Diamond Turning (SPDT) process in which the copper layer is milled down to the requested layer thickness and surface form. 
Fig. 13 Images of the M2 mirror and part of its support spider. Top: The optical (hyperbolic) surface. Bottom: The rear (flat) surface with the cross-hair used to support alignment of the completed telescope with the laser
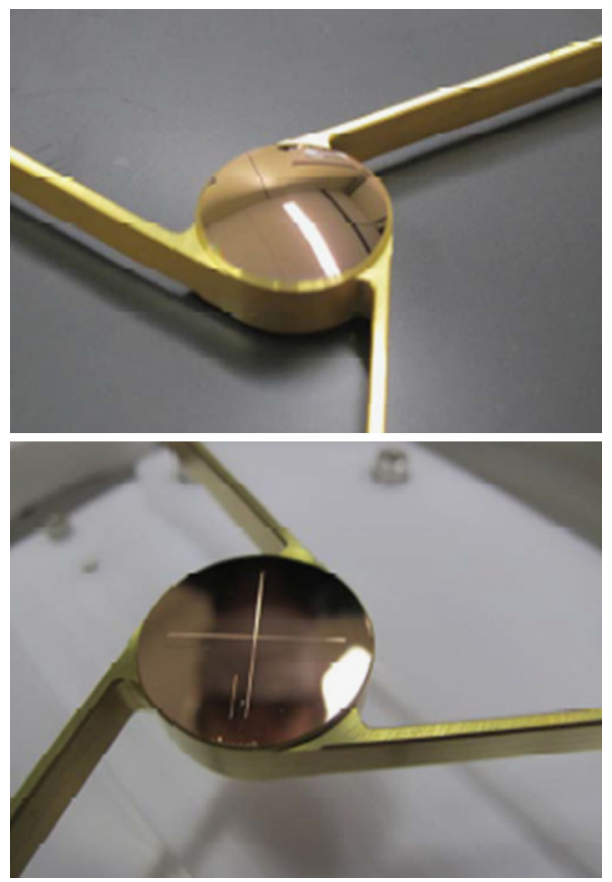

- All surfaces were coated and sealed against corrosion with a metal glass layer deposited on a thin chromium adhesion layer.

- Finally a thin Cr-Au layer (chrome for adhesion and gold for optical purposes) covered completely M1, the M2 bipods and M2. The gold coating was applied to satisfy the optical requirements of reflectivity at the working wavelength as well as over the solar and thermal spectrum.

Major issues were encountered during the qualification of this process. It was recognized in particular that cleanliness of the surfaces prior to coating was absolutely critical to prevent delamination. Baths used for washing had, in all cases, to be with fresh, clean solvent.

M1 has a thickness of $2 \mathrm{~mm}$ which was considered optimum by trading thermal conductivity, stiffness, low mass and manufacturability. It has an active surface in optical quality with a diameter of $206 \mathrm{~mm}$, which is slightly larger than the entrance pupil of $204 \mathrm{~mm}$ to avoid any edge effects (Table 6).

At the location of the intermediate image of the telescope, a field stop blocks all light and all thermal radiation from outside the field of view. The field stop is made of titanium and has a cylindrical shape. It was bonded with three small posts to the rear side of the mirror after axial and lateral alignment.

\subsection{Back-End Optics and Filter}

The instrument operates over both the nightside and the dayside of Mercury. Over the dayside, the reflected light from the surface of Mercury is, of course, intense and hence a narrowband interference filter to isolate the laser wavelength was incorporated into the design. The width of the filter was a delicate trade-off. The temperature inside the spacecraft and at the instrument was expected to be between $-30{ }^{\circ} \mathrm{C}$ and $+55^{\circ} \mathrm{C}$ depending upon the position of 
Table 6 Summary of the characteristics of mirrors, M1 and M2

\begin{tabular}{lll}
\hline Parameter & M1 & M2 \\
\hline Active diameter & $204 \mathrm{~mm}$ & $27 \mathrm{~mm}$ \\
Radius of curvature & $317.053 \pm 0.006 \mathrm{~mm}$ & $41.898 \pm 0.006 \mathrm{~mm}$ \\
Conic constant & $-1 \pm 0.05 \%$ & $-1.6653 \pm 0.025 \%$ \\
& $(\mathrm{sag} \pm 212 \mathrm{~nm}$ at $102 \mathrm{~mm}$ radius) & $(\mathrm{sag} \pm 80 \mathrm{~nm}$ at 13.5 mm radius) \\
Central hole diameter & $2.002 \pm 0.020 \mathrm{~mm}$ & $\mathrm{~N} / \mathrm{A}$ \\
$\begin{array}{l}\text { Coating reflectivity } \\
\text { (solar spectrum) }\end{array}$ & $93 \%$ & $93 \%$ \\
$\begin{array}{l}\text { Coating reflectivity } \\
\text { (thermal IR) }\end{array}$ & $98 \%$ & $98 \%$ \\
Surface roughness & $2.2 \mathrm{~nm}$ & $2.0 \mathrm{~nm}$ \\
\hline
\end{tabular}

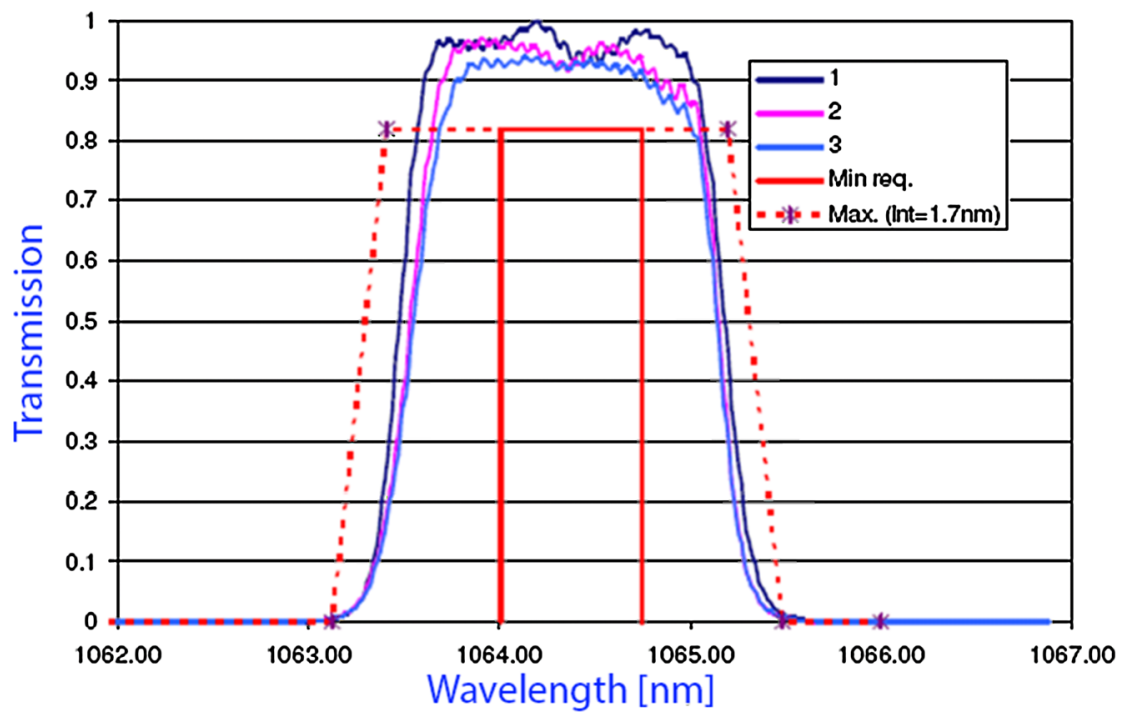

Fig. 14 Maximum (dashed red) and minimum (solid red) widths for the Back-End Optics (BEO) filter and the minimum transmission requirement compared to 3 filters from the batch delivered by Barr Associates

Mercury about the Sun (Mercury has a relatively high orbital eccentricity which produces a strongly varying heat load on the spacecraft) and the errors in the spacecraft thermal model. Variations in temperature affect both the laser emission wavelength $(\sim 4.85 \mathrm{pm} / \mathrm{K})$ and the bandpass of interference filters $(\leq 8 \mathrm{pm} / \mathrm{K})$. It was therefore necessary to select a bandwidth about the Nd:YAG wavelength that was both large enough to cope with the temperature variations at both positions in the instrument and yet narrow enough to eliminate most of the reflected sunlight from the surface. The resulting requirements are indicated in Fig. 14.

Filters were obtained from Barr Associates with a peak transmission of $\sim 92 \%$. Some slight differences within the batch were identified in testing (cf Fig. 14). The filter substrates were of fused silica (ES Fused Silica of Fujitok Corporation) which is intrinsically radiation hard, and were coated with hard-oxides. Subsequent testing showed the wavelength of the 
Fig. 15 Mechanical implementation of the Back-End optics (BEO)

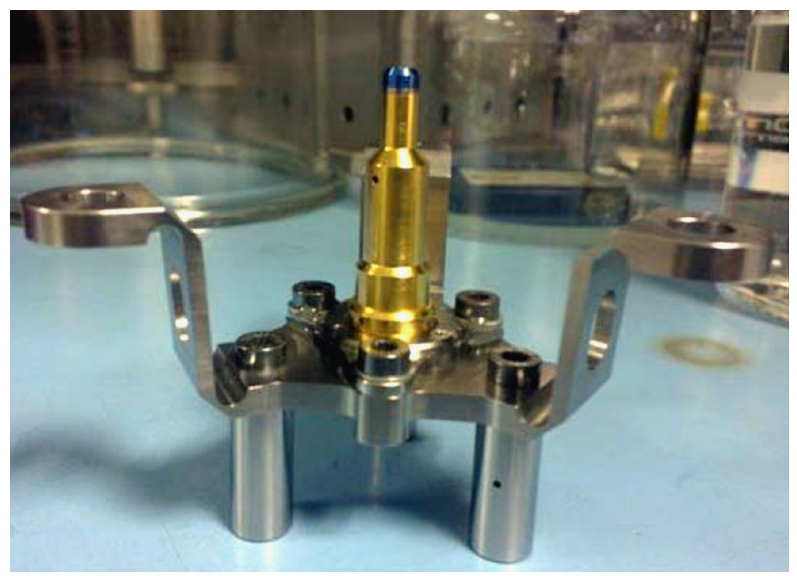

filter transmission to vary with temperature according to the equation

$$
\lambda_{c}=0.0063 T_{0}+c
$$

where $\mathrm{T}_{0}$ is the temperature in ${ }^{\circ} \mathrm{C}$ and $\lambda_{\mathrm{c}}$ is in [nm]. The constant, c, could not be measured precisely (the response curve is close to a top hat function as can be seen in the figure) but is around $1063.85( \pm 0.02) \mathrm{nm}$. The peak transmission of the integrated system (RTL, BEO and filter) was found to be $76.7 \%$ (beginning of life).

The optical elements of the BEO followed the original design concept. The rays come from the image plane of the telescope and enter the first part of the BEO (the collimating objective or COO). From there they pass as a collimated beam through the filter. Collimation assures that deviations of the thickness or the mean refractive index of the real filter have only minor influence on the optical properties of the whole system. The second part of the BEO (the imaging objective, IMO) focuses the light on the avalanche photo diode (APD) behind the window of the APD module in which the APD itself is housed. The required angle of incidence at the filter determines the angular magnification of the COO subsystem.

As pointed out above, although the BEO provides a 1:1 imaging optic and, thus acts a transfer optic, the $\mathrm{COO}$ and the IMO are not symmetrical in order to support the straylight rejection concept. The optical design produces defocusing of only $\pm 9 \mu \mathrm{m}$ over the design temperature range $\left(-20{ }^{\circ} \mathrm{C}\right.$ to $+65{ }^{\circ} \mathrm{C}$ optimized at $20{ }^{\circ} \mathrm{C}$ but including margin) and meets the required radiation tolerance.

The aperture stop of the whole receiver system is located in the collimated beam of the BEO. The position of the aperture stop was adjusted in a way that the entrance pupil is located on the surface of the primary mirror of the RTL. The mechanical parts were coated internally with a low reflection material (PCO22). The visible external surfaces of the housing, which were made of titanium, were gold-plated to support thermal control. The final mechanical implementation is shown in Fig. 15.

\subsection{The Focal Plane Assembly (FPA), the Detector and Its Housing}

The Avalanche Photo Diode-Assembly (APD-A) is the detector assembly that is mounted onto the BEO tripod. Together with the BEO, it forms the Focal Plane Assembly (FPA). APD-A is responsible for the detection of the start and return laser pulses. The start pulse is derived from the transmitter and delivered to the APD-A by redundant optical fibres. In 
Fig. 16 CAD model of the focal plane assembly (the Avalanche Photo Diode-Assembly) showing its main interfaces (thermal, optical and electrical)
Fig. 17 Two APD hybrid modules from one of the flight batches. The APD itself can be seen as the bright round disc mounted within a black square at the centre of the head. The APD is seen through a fused silica window. The head is sealed and contains dry nitrogen
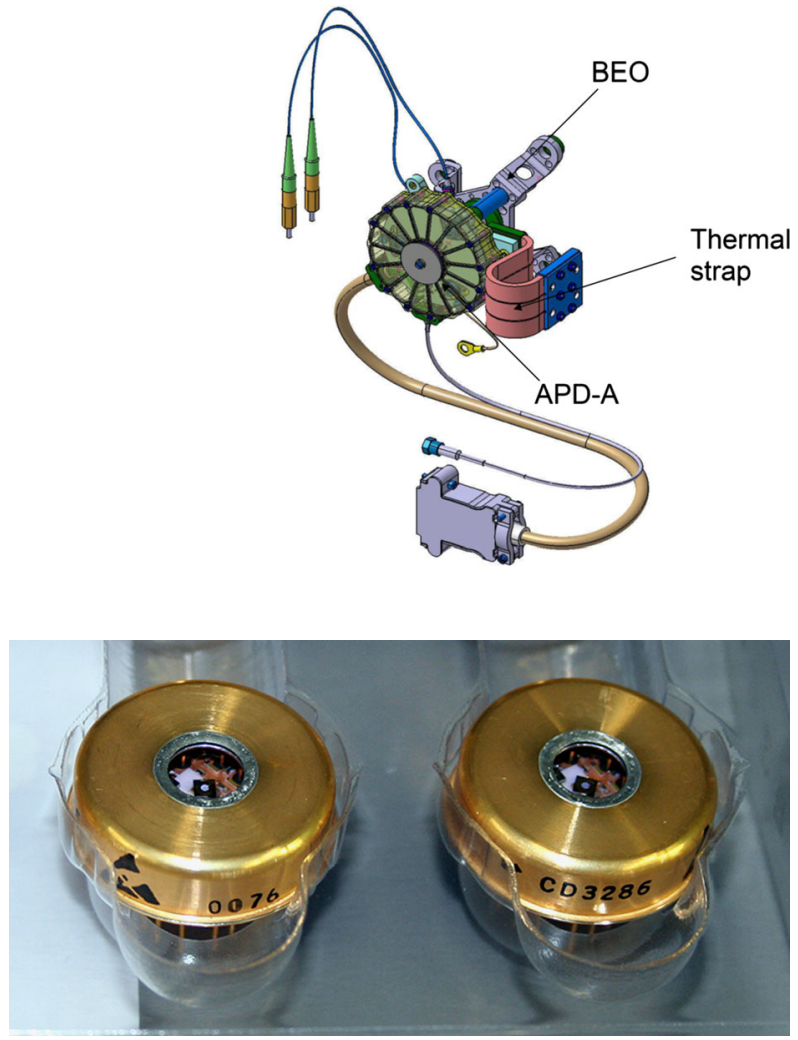

addition to measuring the start time, the start pulse is also used to determine the emitted energy. The return pulse is delivered by the RTL through the BEO.

The main component of the APD-A is the APD which is integrated in a housing referred to as the APD module. This housing also contains a transimpedence amplifier (TIA), a Peltier element and a temperature sensor in order to keep the detector at its operating temperature defined as a set-point by the flight software. The thermal coupling needed to evacuate or deliver heat to the APD is provided through a heat sink made of AlSiC. This heat sink is connected to the spacecraft cold finger via thermal straps (Fig. 16). The module is sealed with a fused silica window.

The APD module was produced Excelitas (formerly Perkin-Elmer) (Laforce 2009). This device (CD 3286) was based on the C30954E APD which has flown, in various forms, in several laser altimeters built by Goddard Space Flight Center for interplanetary flight including MLA, LOLA, and MOLA (Cavanaugh et al. 2007; Smith et al. 2010; Zuber et al. 1992). The device (Fig. 17) contains an infrared enhanced silicon APD that has a quantum efficiency of $34 \%$ at $1064 \mathrm{~nm}$ - an very high value for silicon detectors making it the best choice for this application. The active surface is around in $800 \mu \mathrm{m}$ diameter. It should be noted that the sensitivity of the device is not uniform at high gains (bias voltages) and peaks at the centre of the device. Therefore centering of the returned beam on the APD is of considerable importance. Maps of the sensitivity over the APD were provided by the manufacturer to allow calibration if high bias voltages are used. The mounting concept to ensure good alignment is shown in Fig. 18. 
Fig. 18 Mounting of the Back-End Optics (BEO) to the APD-A to ensure accurate positioning at the focus. The window of the APD module can be seen in purple just to the left of the focus position on the APD itself

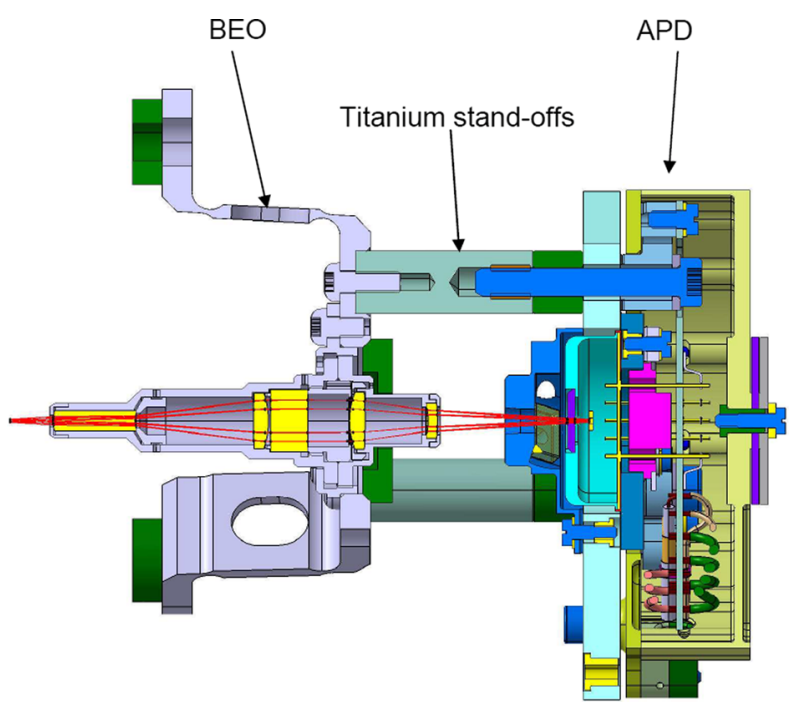

APDs use a multiplication mechanism for generating additional electrical charge carriers that contribute to the photocurrent. The multiplication process is initiated by an external electric field that accelerates the electrons in the conductive band to the positive pole. As a result of the acceleration process, the electrons gain kinetic energy. If the kinetic energy of the accelerated electron is high enough it can eject other electrons by collision. This collision results in a multiplication process which is also called an avalanche process. The magnitude depends on the electrical field across the photodiode and the ambient temperature. Because the electrical field depends on the bias voltage, the avalanche process can be expressed as a function of the applied bias voltage on the photodiode. A higher bias voltage, and therefore a higher electrical field across the photodiode, causes a higher multiplication of the photocurrent. The ratio of the photocurrent at any bias voltage to the photocurrent at a specific bias voltage determines the multiplication of electrical charge carriers as function of the bias voltage (Laforce 2009). Operating the APD at a high bias voltage results in a high multiplication of electrical charge carriers and thus a high electrical current. Because the multiplication process is limited by device properties there is an upper limit for the bias voltage beyond which an uncontrolled fluence of electrical charge carriers is seen. This voltage threshold is called the breakdown voltage and is around $400 \mathrm{~V}$ for these devices at $25^{\circ} \mathrm{C}$. It is, however, highly temperature and device dependent, as shown in Fig. 19. Although the APD hybrid module was fitted with a Peltier element to stabilize the temperature such that a specific voltage could be defined (see below), it was decided to implement a variable high voltage source for the APD as a safety measure. This is provided by a separate analogue electronics board within the Analogue Electronics Unit (AEU).

The APD needs to be operated below breakdown. It was originally intended to operate at a voltage that would provide a responsivity of $1500 \mathrm{kV} / \mathrm{W}$. The measured voltages for the flight module and the noise generated by the device at two different operating temperatures are given in Table 7.

A Peltier element was added to the APD packaging to allow thermal control. In normal mode, the APD temperature can be set between $0{ }^{\circ} \mathrm{C}$ and $25^{\circ} \mathrm{C}$ (cf Table 7). The optimum signal to noise is a complex trade-off between detection efficiency and dark current. Originally, the plan was to reduce the dark current by selecting lower temperatures. However, testing illustrated that the optimum signal to noise has a rather broad maximum and 


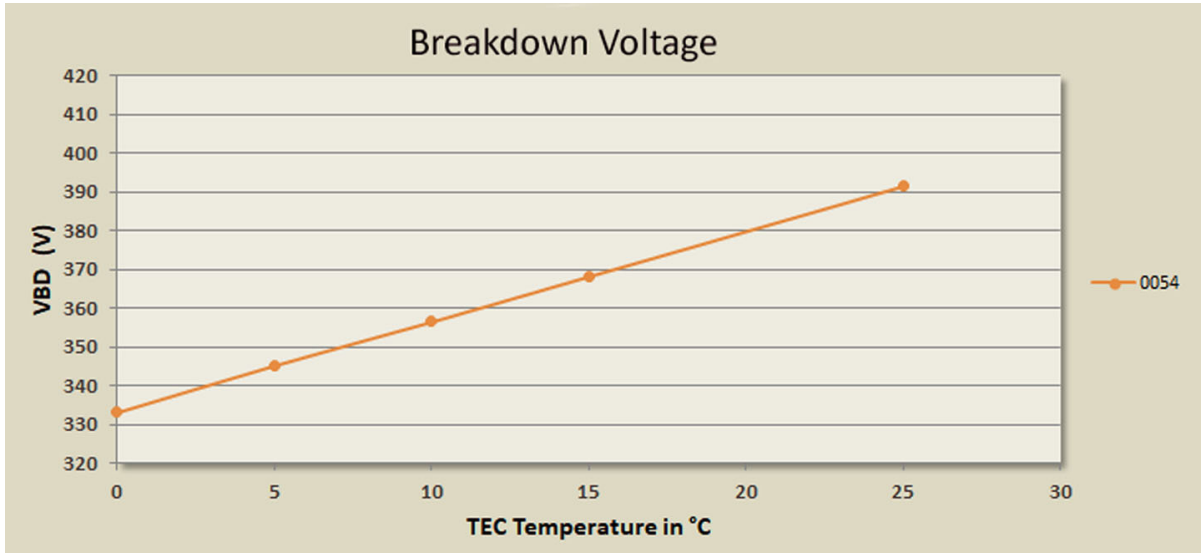

Fig. 19 Breakdown voltages with temperature for the APDs in one batch of APDs as provided by the manufacturer

Table 7 Properties of the flight APD (module \#54) at the two temperature extremes of the thermal control system

\begin{tabular}{lrl}
\hline Property & & Value \\
\hline Breakdown voltage & $25^{\circ} \mathrm{C}$ & $391.5 \mathrm{~V}$ \\
& $0{ }^{\circ} \mathrm{C}$ & $333.1 \mathrm{~V}$ \\
Operating voltage (to & $25{ }^{\circ} \mathrm{C}$ & $354.6 \mathrm{~V}$ \\
give $1500 \mathrm{kV} / \mathrm{W}$ ) & $0{ }^{\circ} \mathrm{C}$ & $303.3 \mathrm{~V}$ \\
Dark Noise Equivalent & $25{ }^{\circ} \mathrm{C}$ & $0.0133 \mathrm{pW} / \sqrt{ } \mathrm{Hz}$ \\
Power & $0{ }^{\circ} \mathrm{C}$ & $0.0157 \mathrm{pW} / \sqrt{ } \mathrm{Hz}$ \\
Illuminated Noise & $25^{\circ} \mathrm{C}$ & $0.2500 \mathrm{pW} / \sqrt{ } \mathrm{Hz}$ \\
Equivalent Power & $0{ }^{\circ} \mathrm{C}$ & $0.3013 \mathrm{pW} / \sqrt{ } \mathrm{Hz}$ \\
Bandwidth & $25^{\circ} \mathrm{C}$ & $54 \mathrm{MHz}$ \\
& $0{ }^{\circ} \mathrm{C}$ & $47 \mathrm{MHz}$ \\
Output voltage swing & & $1.20 \mathrm{~V}$ \\
\hline
\end{tabular}

higher temperatures may actually be preferable. A nominal value of $25^{\circ} \mathrm{C}$ has been chosen for flight. The Peltier can also be used in reverse mode to heat to $>60{ }^{\circ} \mathrm{C}$ to allow for the possibility of in-flight annealing of radiation damage (referred to as Annealing Mode).

The heat conduction between the heat sink and the spacecraft heat pipe system is performed using a thermal strap ( $50 \mathrm{~mm} \times 15 \mathrm{~mm}$ in cross-section). This has to be flexible as there is relative movement between the APD-A and the heat pipe cold finger caused by thermal flexure. In order to provide these two functions and at the same time keep the volume of the strap compact, a thermal strap design using copper foils was selected. Three stacks of foils provided the required conductive coupling and still left enough flexibility for the relative movement between the two connection sides.

The start pulse fibres (manufactured by Diamond) have to deliver the small fraction of the laser pulse to the APD active area. In order to have a stable pulse energy, single mode fibres were selected rather than multi-mode. It was required that a difference in the time delay between the redundant fibres should be $<20$ ps. Hence routing of the fibres had to be consistent with a length difference of $<6 \mathrm{~mm}$ over a length of $1.06 \mathrm{~m}$ (assumed refractive index $=1.51$ ). The AVIM connectors from Diamond were used for fibre-termination. The fibres 
successfully passed radiation testing. The light emerging from the fibres in the focal plane of the receiver has to be delivered to the APD. This was performed using a small mirror within the FPA which was also used to adjust the flux to the APD thereby preventing saturation. It was required that $\leq 7500$ photons per pulse would be passed through one fibre and $13.6 \%$ of these photons should reach the detector (taking into account the mirror reflectivity and tolerances) to initialize the time-of-flight measurement.

In order to deliver the needed high voltage to the APD detector, the APD module is installed in sockets on the circuit card assembly (CCA) which contains the necessary electrical components. Capacitors were used to protect the Transimpedance Amplifier (TIA) of the APD module and a set of resistors were used to improve ripple rejection. The capacitors were selected to have an impedance of $<1 \Omega$ in the frequency range of interest $(10 \mathrm{kHz}-$ $40 \mathrm{MHz}$ ).

The back of the detector is most vulnerable to radiation because in this direction there is the least shielding from the spacecraft structure or other modules on the optical bench. For this reason, shielding with a high density Polyethylene (UHMW-PE) and an AluminiumTantalum-Aluminium sandwich was originally planned to block energetic neutrons. The UHMW-PE was eventually removed because of perceived risks to the APD associated with electrostatic discharge. The position of the instrument with respect to the spacecraft in the cruise configuration also made this additional protection unnecessary

The whole assembly includes the APD-A housing which contains and protects the fore mentioned parts. The constraints of thermal, mechanical and electronics aspects lead to a complex but still compact and rigid design.

\subsection{The Baseplate and the Baseplate Unit (BPU)}

The function of the Baseplate (Fig. 2) is to support the main opto-mechanical components of the transmitter (LHB) and receiver (RTL and FPA), and maintain their co-alignment in the mission environment. The LHB, RTL and FPA are mounted to the Baseplate to form the Baseplate Unit (BPU) which is then mounted to the spacecraft optical bench (SOB). Despite its somewhat primitive appearance, the Baseplate is a vital element in the BELA system.

To cope with the wide temperature range, a sandwich panel design with near-zero CTE CFRP (carbon-fibre reinforced polymer) facesheets and aluminium honeycomb was selected. The Baseplate is mounted perpendicularly to the SOB, where two bolted connections at the outer corners were foreseen. An additional panel, made of the same materials, was bolted to the main panel and provides the third attachment point to the SOB, thus stiffening the whole design of the BPU. As the Baseplate and the SOB were made of the same materials, thermal expansion mismatch did not need to be considered. This allowed hardmounting the BPU to the SOB, which facilitated the fulfillment of the stiffness requirements. For alignment purposes, shims and oversized holes were foreseen. Two pairs of spherical washers at each interface prevented distortions of the structure during the integration to the spacecraft. MJ55 carbon fibers were selected for their stiffness, while a cyanate-ester matrix was used to minimize the moisture absorption and the related expansion of the composite material when exposed to humid atmosphere and subsequently to vacuum.

\subsection{The Analogue Electronics Unit (AEU)}

The Analogue Electronics Unit provides a programmable high voltage (250 V-450 V) to the APD-A, via a switching converter (variable gain amplifier with a precision of $0.625 \mathrm{~V}$ ) 
and amplifies the APD-A output (carrying start and return pulses) with low noise and programmable gain (approx. $4 \mathrm{~dB}$ to $44 \mathrm{~dB}$ ). The system runs from $+12 \mathrm{~V}$ and $\pm 5.2 \mathrm{~V}$ supplies consuming $73.5 \mathrm{~mA}$ and can operate over a temperature range of $-25^{\circ} \mathrm{C}$ to $+55^{\circ} \mathrm{C}$. The programmable gain is implemented through discrete values of a gain code which provides steps of $<3 \mathrm{~dB}$ in the amplification from gain code 0 to 15 . The output signal is then passed to the rangefinder module (RFM) that is housed within the ELU.

Mechanically, the AEU comprises two PCBs mounted within one frame. The aluminium box is closed by two covers and is mounted using three titanium blade-like feet to ensure thermo-elastic decoupling from the spacecraft platform. All the aluminium components were treated with Alodine 1200 to guarantee electrical coupling. The unit weighs $380 \mathrm{~g}$.

\section{The Aperture Baffling Systems}

\subsection{The Receiver Baffle Unit (RBU)}

The concept and implementation of the Receiver Baffle Unit (RBU) have been discussed in two previous publications (Seiferlin et al. 2007; Beck et al. 2011) and we provide only a brief review here.

\subsection{Baffle Concept}

Because of the high solar flux at Mercury, equivalent to 10 solar constants at perihelion, the use of an absorbing baffle to reduce straylight was excluded from the beginning. From several reflective design options, the Kleinhans/Stavroudis concept was chosen (Kleinhans 1976; Stavroudis 1993). This consists of an alternating sequence of ellipses and hyperbolas which minimizes the number of reflections necessary to reject the light back through the front aperture. By knowing the required inner diameter and the minimum incidence angle of the Sun, the length of the baffle can be determined. The number of elliptical and hyperbolic sections determines the outer diameter. The BELA RBU is divided into six segments, usually referred to as baffle vanes, each comprising an elliptical and a hyperbolic section. This approach was a result of considering the manufacturing aspects. The clear aperture and length are $211 \mathrm{~mm}$ and $315.6 \mathrm{~mm}$, respectively, as shown in Fig. 20.

Modelling of the geometry was presented by Beck et al. (2011) and the results for perfect surfaces (i.e. perfect reflection) are shown in Fig. 21.

\subsection{Baffle Implementation}

The RBU consists of a main body suspended on titanium bipods to provide mechanical and thermal decoupling from the spacecraft panel. The main body is a stack of segments assembled using bolts. The first one is a simple disc which acts as a field stop, while the others comprise the elliptical and hyperbolic sections. This arrangement allows machining a very sharp transition edge, which is of paramount importance to minimize straylight. The interface to the spacecraft MLI is provided by the so-called front ring which has enough overlap to prevent solar radiation being trapped in the spacecraft cavity. The front ring is mounted via thermally insulating flexures to the main body and has a low-emissivity coating to reduce the radiative coupling. The RBU must be insulated from the spacecraft cavity to limit conductive heat input to the spacecraft. Two concentric gold plated titanium foils separated by spacers insulate the front section, while a conventional MLI blanket is mounted to the 


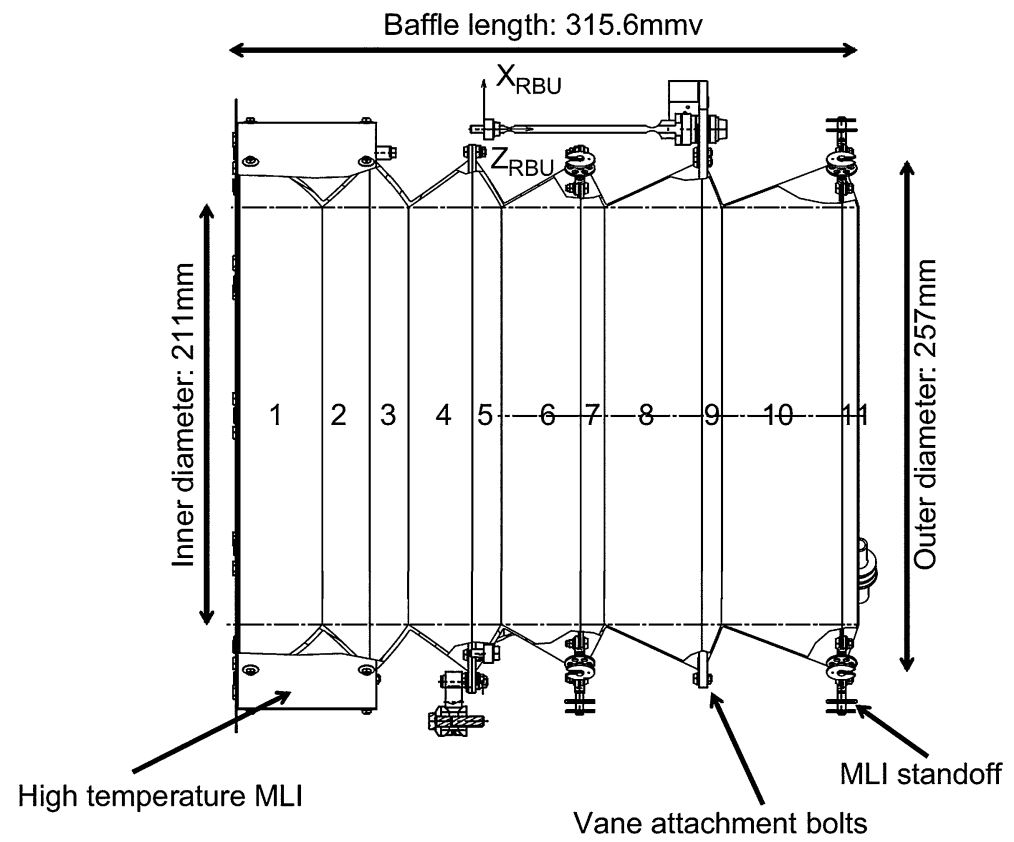

Fig. 20 Geometry of the Receiver Baffle Unit (RBU) for BELA

Fig. 21 The results of a Monte Carlo ray-tracing simulation of the BELA baffle. The solid line represents the reflections per reflected ray and the dashed line shows the percentage of rays that are reflected out of the baffle aperture. (From Beck et al. 2011)

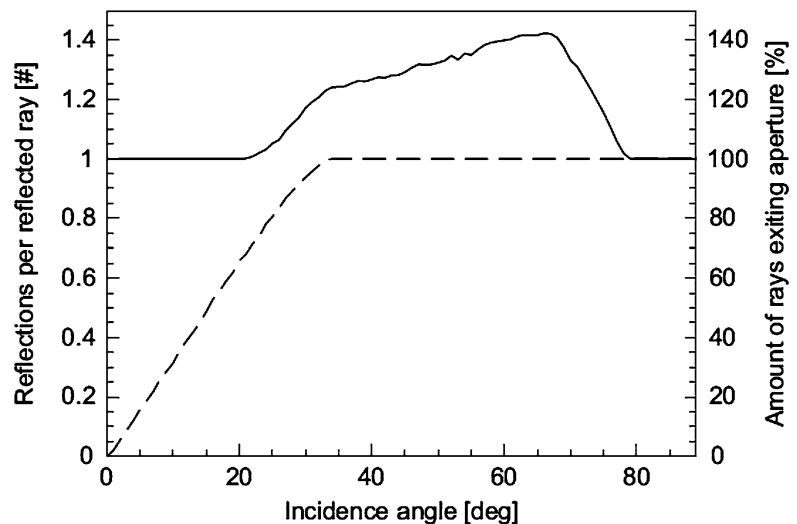

rear section. Anti-contamination heaters were mounted to be used during cruise to keep the RBU slightly above the spacecraft temperature thus preventing condensation of organic substances. Molecular contamination would react with the mirror surfaces when exposed to intense UV radiation and compromise the thermo-optical properties. For the on-ground operations, a protective cover and a purge line were also foreseen to protect the inner cavity of the RBU. For the purpose of co-aligning the RBU to the RTL, optical references were mounted to the RBU. The total mass of the RBU including pigtails for electrical connections and thermal straps for the connection to the spacecraft is $2.150 \mathrm{~kg}$.

After having defined the basic requirements, materials and manufacturing technologies were traded-off. The selected candidates were electroformed nickel, aluminium with diamond machined mirror surfaces, and AlBeMet with mirror surfaces machined onto a thick 
(350-500 $\mu \mathrm{m})$ pure copper layer selectively deposited on the inside of the vanes. Electroformed nickel was rejected because of inadequate thermo-optical properties, the moderate thermal conductivity, and the high density, which would have led to a design that would have both exceeded the available mass budget and failed to fulfil the heat rejection requirements. AlBeMet would have had outstanding properties for this application. Its specific stiffness is second only to pure beryllium and the CTE is moderate, $14 \mathrm{ppm} / \mathrm{K}$ compared to $24 \mathrm{ppm} / \mathrm{K}$ for aluminum. The thermal conductivity is the same range of many aluminium alloys, while the specific heat is significantly higher due to the beryllium content of $62 \%$. The resulting design would have been a very lightweight structure showing low distortions when exposed to variable thermal loads. However, the necessary technological effort for the production of the internal mirror surfaces was deemed excessive and led to the decision to reject this promising solution. As experienced during the development of the receiver telescope, selective copper plating of beryllium and its alloys is a challenge in terms of process reliability. Applying the final high-reflectivity coating on the strongly curved inner surfaces is also a non-trivial engineering task. Aluminium was finally selected as the best material. In the first development phase, the thermo-optical properties of several alloys were investigated, in the course of which diamond machined samples underwent thermal cycling and UV radiation exposure to verify the compatibility with the expected environment. Al 6082 was chosen by considering its machinability, strength, thermal conductivity, and availability. Furthermore, as with all the other tested alloys, no degradation could be observed after the environmental tests. UBE worked in close cooperation with LT-Ultra, the company selected for the diamond machining of the inner mirror surfaces, to define the whole machining sequence of the baffle segments. In the frame of this work a further optimization step was undertaken, whereby the heat treatment of each single segment was tuned to maximize the thermal conductivity while retaining sufficient strength to remain compatible with the results of the FE analysis. A mechanical qualification model, the so-called M-EQM, was produced to verify in advance the strength and stiffness requirements.

An attempt to further improve the performance consisted in depositing a multilayer dielectric coating on the mirror surfaces. This had been indicated as being promising in a paper study conducted by ESA. However, this attempt failed because of the strongly curved geometry of the vanes, which compromised the homogeneity of the thicknesses of the single layers. Despite the custom development of a complex two-axis rotating support to try to produce homogeneous uniform layers within a vapour deposition chamber, the coatings were not sufficiently uniform and this severely affected the overall coating properties. Consequently, the diamond machined mirror surfaces remained uncoated and were protected solely by a natural oxide layer.

\subsection{Front Ring Production}

Being exposed to a more severe thermal environment, as well as to higher risk of contamination and mechanical damage, different materials were chosen for the production of the front ring. Titanium was selected as the substrate because of its moderate thermal expansion, strength, and availability as thin sheet metal. White ceramics (alumina and zirconia) were identified as protection for the external surface against the extreme heat from the Sun. Layers with different thicknesses were deposited by plasma-spray on sandblasted samples, which were exposed to UV radiation and thermal cycling to assess the degradation of the thermooptical performances. After thermal cycling between $-80{ }^{\circ} \mathrm{C}$ and $+400{ }^{\circ} \mathrm{C}$ all samples were intact, thus demonstrating sufficient adhesion of the layers to the substrate. Although the thermo-optical properties of aluminium oxide are initially slightly superior, zirconium 
oxide showed better performances after exposure to UV and molecular contamination. The final configuration was therefore a roughly $100 \mu \mathrm{m}$ thick zirconia layer applied on a $0.5 \mathrm{~mm}$ thick titanium Grade 5 substrate. Zirconia has the further advantages of being less thermally conductive than alumina, and having the same thermal expansion as the titanium substrate. The titanium substrate was sandblasted on both sides to minimize warping and the side facing the spacecraft was gold sputtered to minimize the radiative thermal coupling to the baffle main body.

\subsection{Thermal Behaviour of the RBU}

Modelling of the thermal behavior of the RBU is challenging because of the shapes of the surfaces. At the time, the thermal modelling software, ESARAD, did not support the required geometries which therefore had to be approximated. The BELA baffle geometrical mathematical model was finally approximated with 40 cones per ellipsoidal and hyperboloidal surface as a trade-off between accuracy and computational time. The thermal nodes from which the temperatures and fluxes were averaged for the lumped parameter method were evenly distributed around the baffle axis. The optical surfaces were modeled with 12 nodes each. The flanges combining the baffle surfaces were also modelled with 12 nodes each. Testing for model correlation was performed using a wide-beam solar simulator, custom-built for the purpose (Thomas et al. 2011). A novel method using Adaptive Particle Swarm Optimization (APSO) was used to make the correlation between the model and the thermal balance tests (Beck et al. 2011). The determination of thermal balance test temperatures was correlated to an accuracy of $4.2^{\circ} \mathrm{C} \pm 3.2^{\circ} \mathrm{C}$, over a temperature range of about $200{ }^{\circ} \mathrm{C}$ under solar as well as infrared irradiation. Given the difficulties in modelling the shapes of the surfaces, this was considered an extremely good result.

As part of the project's contribution to technology transfer, the manufacture of the flight unit was transferred to an SME (small medium-sized enterprise, Almatech) which also supported finalization of the design.

\subsection{Protection of the Laser}

To minimize environmental influences (radiation, heat, contamination with particles) on its properties, the transmitter needs to be protected. The Transmitter Baffle Unit (TBU) and the Straylight and Contamination Protection Unit (SPU) were developed for this purpose.

\subsection{The Transmitter Baffle Unit (TBU)}

The purpose of the TBU is to minimize the environmental heat fluxes entering the MPO through the BELA transmitter aperture. In particular, the TBU was required to block direct sunlight, the sunlight reflected by the planet, and the planetary IR-flux from reaching the $\mathrm{BEX}$ and the MPO payload support structure. While rejecting the environmental fluxes into deep space, the TBU needed to be completely transparent to the laser beam $(\lambda=1064 \mathrm{~nm})$ itself and should not obstruct the outgoing beam.

The MPO orbit is designed with a minimum sun angle of $38^{\circ}$ with respect to the boresights of the receiver and transmitter. The TBU is composed of two main elements, a reflective Stavroudis baffle and a bandpass filter. The baffle reflects the direct sunlight back into deep space. The radiated IR flux from the planet cannot be blocked in the same way because BELA will be nadir facing. Therefore a filter has been implemented to protect the laser. The filter blocks both the radiated and reflected fluxes from the planet (Fig. 22). 
Fig. 22 Transmitter Baffle Unit (TBU) design with the front-ring (purple transparent part) facing towards the planet. The

Stavroudis baffle (blue parts) reflects the incident light, the filter (grey part) is on the back end of the baffle and mounted in the filter mount (dark purple)

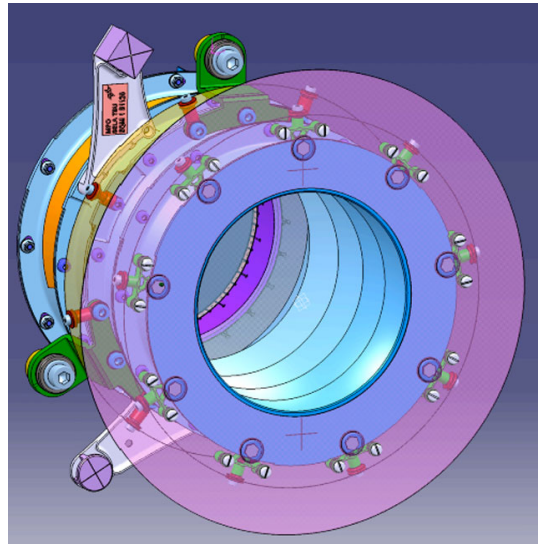

Fig. 23 The Stavroudis baffle of Transmitter Baffle Unit (TBU).

The reflection of the photographer can be seen (inverted) at the top of the baffle

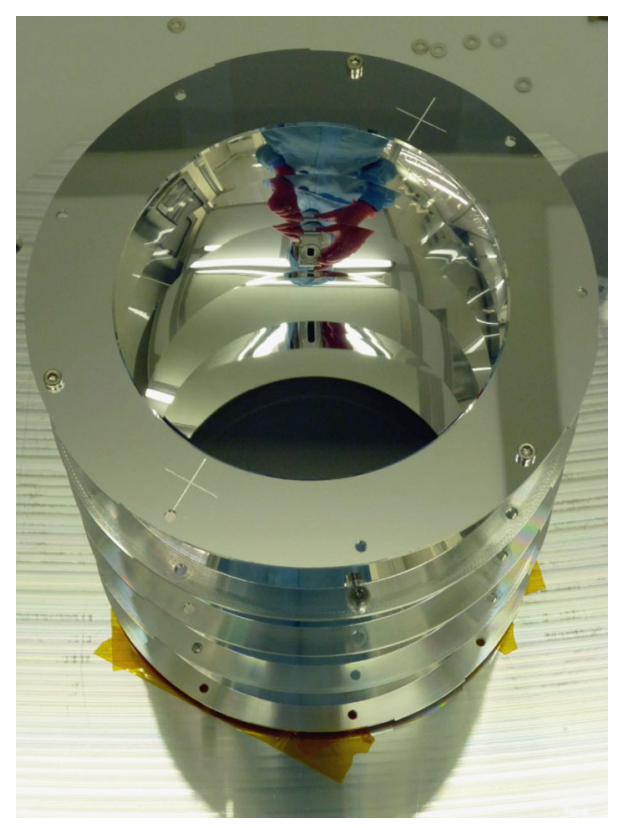

The working principle of the Stavroudis baffle has been described in the previous section. The implementation for the TBU consists of several vanes (segments) made out of optical grade Aluminium RSA-6061-T6 (Fig. 23). This quality provides a surface roughness of $<3 \mathrm{~nm}$ when diamond machined. The thicknesses of the vanes were constrained by thermal, optical and structural needs and is in the range of 1.2 to $1.8 \mathrm{~mm}$. There were no coatings on the inner or outer side of the Stavroudis shape.

The filter consists of a $5 \mathrm{~mm}$ thick Sapphire substrate with a dielectric coating on the front side (towards the planet), and an anti-reflection coating on the back side (towards the laser beam expander). The filter is glued into a filter mount made out of Titanium Ti6Al4V. The Stavroudis baffle and the filter mount are connected via a tight fitting clamp in order to achieve a high structural stiffness and limit the vibration and shock loads transferred to the filter mount assembly. 
The isostatic mounts of the TBU are made of titanium to minimize the conductive flux from the TBU to the baseplate and to ensure a sufficient structural stiffness. The front part of the baffle facing the spacecraft MLI is wrapped in gold-coated titanium foil.

Although optimized for its purpose, the efficiency of the Stavroudis baffle is limited by several manufacturing and degradation aspects, which are taken into account in the TBU design. The specular reflectivity of the baffle is reduced by scatter losses, mainly due to the surface roughness and the rounding of the edges between the vanes. Moreover, the surface will deteriorate because of contamination and environmental degradation (VUV, UV, high temperature, high energy particles). The thermally induced distortion of the Stavroudis shape can lead to a further worsening of the baffle's efficiency because of modification of the geometry. Extensive solar irradiation tests of material samples of the Stavroudis shape were performed at ESTEC and showed no significant degradation. The results of those tests have been incorporated into the thermal-mathematical simulation of the TBU.

The front ring is directly exposed to space and is subject to environmental degradation; the combination of high temperature and solar radiation being the main causes of degradation. The applied ceramic coating is able to withstand these fluxes without significant degradation, as tests at ESTEC have shown.

The filter is also affected by environmental degradation and contamination. A cruise heater is positioned on the filter mount to reduce contamination.

For the thermal design of the TBU, a fully passive thermal control system with the advantage of low mass and complexity was chosen. This means that the thermal behavior of the unit is strongly coupled with its optical design. Because of the harsh environment all exposed surfaces are subject to degradation. Therefore, coatings have been avoided whenever possible.

Very high temperatures are expected on the external side of the TBU. To isolate the system from these temperatures and to avoid direct heat fluxes onto the baffle front section, a titanium front ring was implemented on the external side as a shield. The material chosen here was the titanium alloy Ti6Al4V. On the outermost nadir spacecraft surface, the gap between the Stavroudis shape and the MLI is closed by this front ring. Following the RBU design, a ceramic high reflectance coating was deposited on the outer (planetary facing) surface of the front ring. This coating (Yttrium-Stabilized Zirconia) provides lower solar absorption. The front ring is well insulated from the baffle. The front ring therefore gets much hotter than the baffle but still well below the working limit of titanium and the applied coating.

The performance of the TBU in the operating environment was evaluated by performing detailed numerical analyses. The thermal model was correlated with the results of the STM thermal balance tests. The analysis shows that during orbit the rejection efficiency of the TBU is between $92.5 \%$ and $96.5 \%$. The sunlight entering is limited to $<0.5 \%$. The heat flux towards the laser is $<0.1 \mathrm{~W}$ and the maximum flux towards the spacecraft is $8 \mathrm{~W}$.

Between the front ring and the spacecraft MLI there is a gap to allow the MLI blankets to expand and adjust. As a consequence, environmental radiation may enter the system leading to a hot cavity. To protect the baffle and the rest of the unit from the effects of this gap, a gold coated titanium foil was incorporated into the design. The TBU is mounted to block inserts on the spacecraft as depicted in green in Fig. 24. Shims and spherical washers are used for the alignment with respect to the outgoing beam.

\subsection{The Straylight and Contamination Protection Unit (SPU)}

The purpose of the Straylight and contamination Protection Unit (SPU) is to protect the outer surface of the beam expander front lens and the inner surface of the TBU filter against 
Fig. 24 Transmitter Baffle Unit (TBU) and Straylight \& contamination Protection Unit (SPU) on the right as mounted on the spacecraft

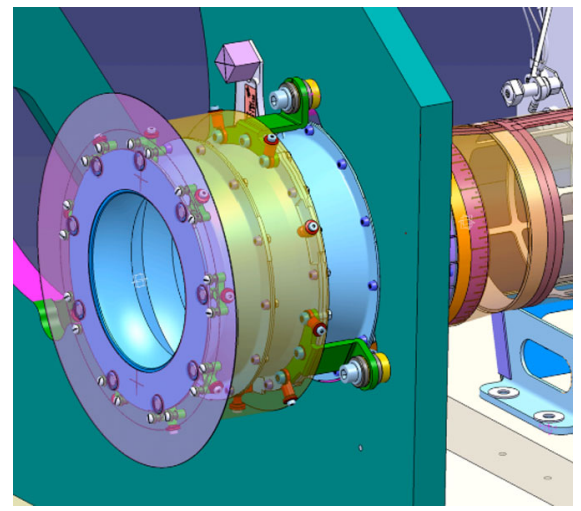

Fig. 25 CAD drawing of the Straylight \& contamination Protection Unit (SPU)

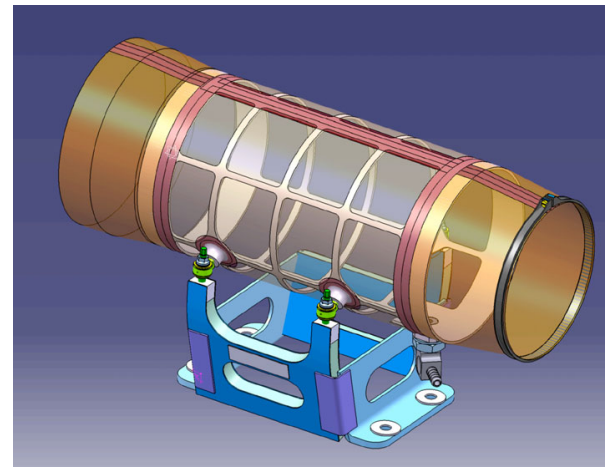

molecular and particulate contamination. The SPU effectively isolates the laser beam on its path towards the aperture in the spacecraft panel and prevents scattering of laser light, that originates from diffuse reflections at the lens/filter surfaces, from entering into the internal spacecraft structure. Furthermore, it supports the limitation of incident light on the laser.

The SPU consists of a cylindrical body connected flexibly on one side to the beam expander of the laser head and on the other to the TBU filter mount (Fig. 25). A support structure fixes the SPU to the spacecraft structure and a purging line is used to limit contamination.

The SPU does not dissipate heat and is not directly exposed to the outer environment. Its temperature is determined by the surrounding elements - primarily by the TBU filter and the BEX. Being mounted on the SOB, a low emittance finish is applied on the outer side to reduce radiation into the spacecraft vane and in particular towards the SOB. The flexible connections to the filter mount and to the beam expander are made of aluminized Kapton foil. The foil is connected to the appropriate structure by the use of clampbands. The tube of the SPU consists of a grid made of ELEKTRON WE43, a high strength magnesiumbased alloy. The grid is also covered by aluminized Kapton foil. The temperature reached by the SPU during the whole mission does not provoke any concern for the materials and the coating used. To avoid contamination of the beam expander front lens and of the TBU filter, the inner side of the SPU uses very low outgassing materials. Instead of applying a coating on the inner side of a bulk cylinder, a Kapton foil is wrapped on a grid. The structural parts and the foils facing the filter and the beam expander have been baked at $80{ }^{\circ} \mathrm{C}$ for three days in order to remove residual contaminants, process contaminants, and handling contaminants. 


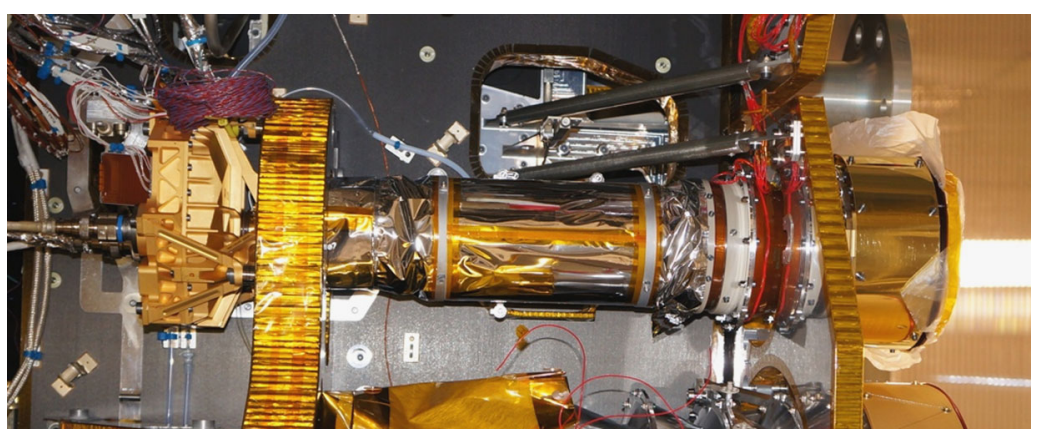

Fig. 26 Laser head (left), Straylight and contamination Protection Unit (SPU) (center) and Transmitter Baffle Unit (TBU) (right) mounted on the Mercury Planetary Orbiter (MPO)

The support structure of the SPU is mounted on the SOB and can be aligned in lateral direction. The grid is mounted with three points to the mount and can be aligned to the boresight axes of the LHB and TBU. The SPU tube was oversized with respect to the laser beam diameter $(85 \mathrm{~mm})$ by $15 \mathrm{~mm}$. This allows a fairly easy integration procedure with adequate accuracy. The flight model TBU and SPU units mounted on the spacecraft are shown in Fig. 26.

\section{The Electronics Unit}

The electronics unit comprises 5 boards ( 3 functional boards with 2 being redundant) that comprise "planes" within the mechanical design allowing independent production.

\subsection{The Digital Rangefinder}

The rangefinder module (RFM) provides

- Tx/Rx pulse detection (the primary task)

- thermal control of the APD (i.e. operating the Peltier element in the APD)

- high voltage set-point generation and control for the APD

- set-point generation and control of the AEU Variable Gain Amplifier (VGA)

- the telecommand/telemetry (TC/TM) interface towards the DPM, i.e. configuration of the RFM for the range window setting

- and the AEU power interface.

The RFM can be operated in 4 different modes as indicated in Table 8.

The RFM digitizes the analogue signal coming from the APD using two analogue to digital converters (ADCs). The ADCs are implemented with a high voltage resolution (12 bits) in order to be able to detect low signal variations. At the time of development, spacequalified 12 bit ADCs could only be procured with $40 \mathrm{MHz}$ clock frequency and hence 2 ADCs were multiplexed to give $80 \mathrm{MHz}$ effective which corresponds to one sample in $12.5 \mathrm{~ns}$. In order to be able to resolve very short pulses, the analogue signal first undergoes a low pass filtering (9.5 MHz cut-off frequency) which results in pulse broadening of the short pulses and reduces noise

A time of flight measurement resolution of $12.5 \mathrm{~ns}$ would correspond to a height resolution of 1.9 metres. This resolution is called here "coarse" measurement resolution. However, 
Table 8 The different modes of the RFM and their purposes. This is expanded further in Table 13 as these operational modes of the RFM effectively define the operational modes of the system

\begin{tabular}{ll}
\hline Mode & Purpose \\
\hline $\begin{array}{l}\text { Test } \\
\text { Albedo }\end{array}$ & $\begin{array}{l}\text { Test mode } \\
\text { Complete sensor signal samples acquired by the RFM without detection of a Tx pulse (to be } \\
\text { used in the event of laser failure to provide surface albedo measurements). }\end{array}$ \\
Sensor & $\begin{array}{l}\text { Complete sensor signal samples (Tx or Rx range window) acquired by the RFM, where the Rx } \\
\text { range window sampling is carried out at a prescribed time delay with respect to the Tx pulse. } \\
\text { Pulse }\end{array}$ \\
\hline
\end{tabular}

the RFM is designed to provide a time of flight measurement accuracy which is better than the coarse resolution. This is done using a set of basis functions which are stored in the built-in field programmable gate array (FPGA) from Actel (model RTAX2000S). The pulse detection is then performed using a matched filter approach on the digitized signal. The signal is convolved with a set of predefined basis functions in order to find the sample bin where maximum signal occurs. Let us look at an example.

In the simplest case, one can assume a Gaussian-shaped return pulse as a basis function and define it as

$$
f(\delta)=\exp \left(\frac{-(t-\delta)^{2}}{4 \ln 2 \sigma^{2}}\right)
$$

where $\delta$ is a sub-sample offset within a range of \pm 0.5 sample and $\sigma$ is the full width half maximum of the Gaussian. The digitized signal from the ADCs is compared with the basis function to obtain the detailed pulse parameters ( $\delta, \sigma$ and pulse energy). Essentially it is performing a pulse fitting on the signal which leads to a so-called "sub-sampling" resolution which is better than $12.5 \mathrm{~ns}$.

The interesting element of the RFM is that basis functions can be defined in an arbitrary way. In order to have an efficient pulse fitting, at a $10 \mathrm{~Hz}$ repetition rate, the arbitrary fitting functions are represented as bi-quadratic polynomials in $\sigma$ (pulse width) and $\delta$ (subsampling offset). In other words, if the arbitrary function can be defined in such a way that it results in having two free parameters, the RFM FPGA code can determine the best fit to the data that exists for that function. The output parameters are referred to as BFOs (basis function filter output) coefficients which are then sent to Earth. Using the BFOs and the programmed basis functions, the time of flight at sub-sampling resolution as well as pulse parameters (pulse width and pulse energy) can be recalculated on ground.

The major advantage of this implementation lies in the possibility of defining arbitrary basis functions. These functions can be programmed in accordance with the pulse forms which are expected to be obtained from the Hermean surface taking into account the shape of the outgoing laser pulse. This has been fully implemented in the RFM and is a proprietary element of the instrument owned by Thales-Alenia Space Switzerland (formerly part of RUAG Space AG) - see Patent No. EP1912078B1 "Laser altimeter, method for range finding and space craft comprising such a laser altimeter".

The fast implementation in the FPGA allows the determination of up to four possible returns within one range window. This was foreseen for long range imaging when the signal to noise may be low. The additional information may provide support to reduce the PFD by on-ground analysis.

The RFM also implements the concept of the range window. The range window is centred around an estimation when the return pulse will be detected. The usual round trip time 
should be between $2.6 \mathrm{~ms}$ and $10 \mathrm{~ms}$, depending on the spacecraft altitude. During the design phase, it was shown that the RFM would not be able to analyze the coarse samples $(2.6 \mathrm{~ms} / 12.5 \mathrm{~ns}=208,000$ samples $)$ of the whole round trip time because the system could not be fast enough. Hence, a shorter range window is analyzed. The window length is $200 \mu$ seconds (about 16,000 samples) and the time to open the window is estimated by a range tracking algorithm that runs on the DPM. The behaviour of the range tracking algorithm can be tested independently from the RFM because it resides in the flight software. The algorithm makes use of the ranging from the last pulses as well as from altitude information delivered by the spacecraft. This altitude information from the spacecraft is coarse but also allows a rapid start-up of ranging if the scanning to find the return pulse using BELA's internal software is considered too slow. Once BELA is ranging, the range window is set such that the return pulse arrives in the middle of the window.

A major advantage of analyzing only a short window is that the possibility of detecting the real pulse is enhanced because the chance of noise spikes being incorrectly interpreted as a return pulse is reduced. Reduction of the analysed part of the range window to a minimum of $2 \mathrm{~km}(\sim 550$ samples $)$ is implemented in software to further decrease the probability of false detection.

Physically, the electronics of the RFM is located on two Circuit Card Assemblies (CCA), the CCAa and CCAb. The CCAa houses mostly digital and power-handling components, while the analogue circuits are mounted on CCAb. The two boards are connected via a rigid-flex-print CCAc and high density connectors on each board. A top level block diagram is shown in Fig. 27.

The mechanical design is based on the overall ELU design. Its mechanical performance was however analysed and eigenfrequencies determined for completeness. The main goal of the thermal design was to keep the temperature below the maximum derated junction temperatures of the active parts, especially for the FPGA and its voltage regulator which have the highest dissipation values on the CCAa. Measures taken to provide good thermal connections were:

- gluing of the FPGA onto the board (underfill)

- provision of as many copper layers as possible nearly filled to $100 \%$, while avoiding direct connection to the guard/chassis ring

- thermal pathways below the FPGA for heat conduction into the mechanical structure

- placement of voltage regulator and FPGA as close as possible to mounting holes.

Three major blocks provide the RFM functionality (see Fig. 27). The ELU interface block receives power from the PCM and control signals from the DPM. The interface allows power bus switching for redundancy and includes an inrush current limiter for all but the $12.6 \mathrm{~V}$ line, diode cross-strapping, and an LVDS (Low Voltage Differential Signaling) data interface using Aeroflex receivers. During the design phase of the RFM, it has been identified as a necessity to provide the lowest possible power supply source impedance to the individual components. As a general rule of thumb, a source impedance of $1 \Omega$ or less is desirable over a frequency range from $\mathrm{DC}$ to $1 \mathrm{GHz}$.

The FPGA block includes the FPGA itself, an $80 \mathrm{MHz}$ master clock, a reset circuit and a level shifter to match $5 \mathrm{~V}$ logic levels with the $3.3 \mathrm{~V}$ FPGA input/output voltage.

The AEU interface includes housekeeping acquisition, the high voltage set-point generator for the APD, the Peltier driver to stabilize the APD temperature, an analogue input stage for the signal input that provides $50 \Omega$ differential-mode impedence and common mode impedence at $>50 \mathrm{k} \Omega$, and analogue to digital converters (ADCs) deliver the digitized pulse data to the FPGA. An input buffer stage has been implemented followed by a 


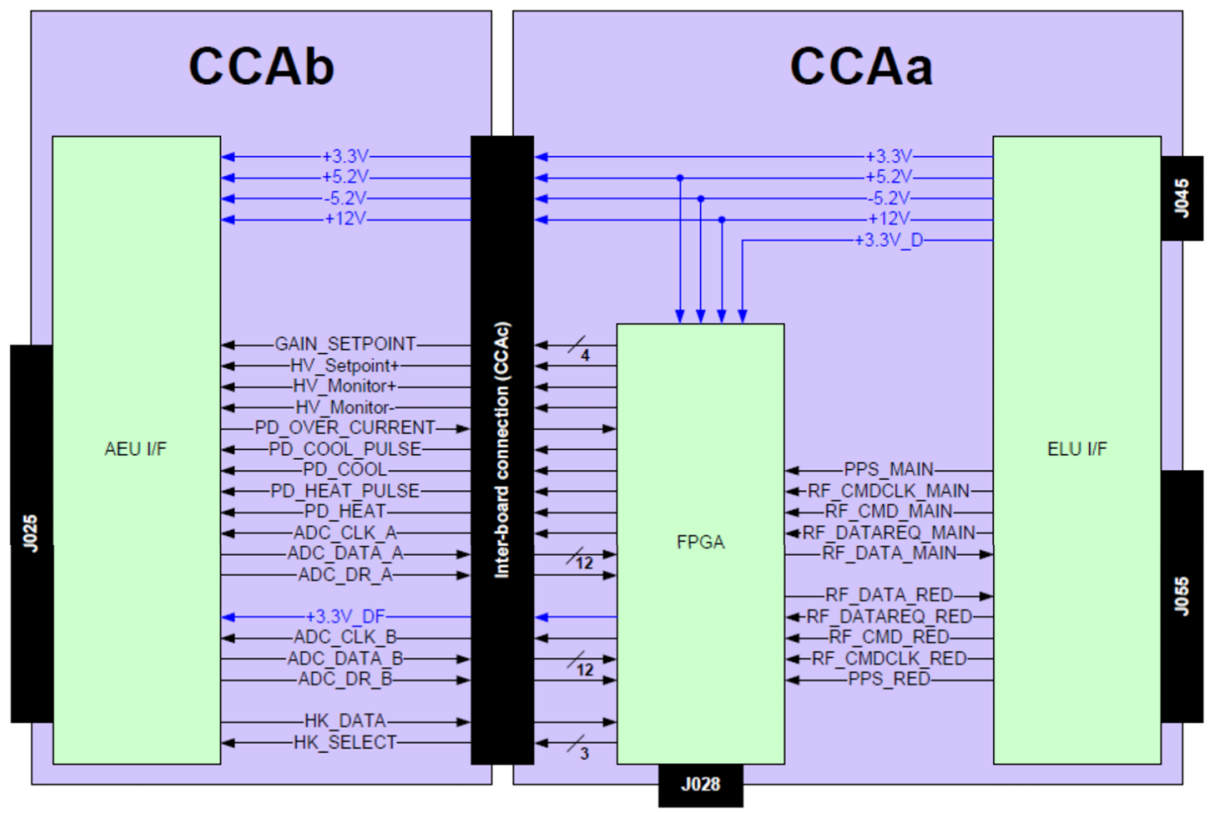

Fig. 27 Top level block diagram of the Range Finder Module (RFM) showing the interfaces to the Field Programmable Gate Array (FPGA) that controls the rangefinder algorithm. The diagram shows how the Electronics Unit (ELU) (to the right) provides main and redundant signals to the FPGA for the Pulse Per Second (PPS) signal and the data request commands while it receives back data from the FPGA. The FPGA in turn sets the parameters for the Analogue Electronics Unit (AEU) (e.g. gain, High Voltage $(H V)$ set-point, Peltier control, and the Analogue-Digital Converter (ADC) parameters) while receiving data and housekeeping from the detector and analogue electronics

differential-to-single ended converter and a second order low pass filter. The purpose of this filter (which is important for the data analysis) is to prevent aliasing effects in the ADCs. It has a Butterworth transfer characteristic with a $3 \mathrm{~dB}$ cut-off frequency of $\sim 27 \mathrm{MHz}$. The analogue input stage is made of three LM6172 operational amplifiers. These devices were selected because of their large bandwidth $(70 \mathrm{MHz})$ and relative ease of use. Nonetheless, during breadboarding experiments, a number of issues had to be resolved to achieve the desired performance. For example, the amplifier chain without lowpass filter already had a noticeable transmission loss at frequencies of $15 \mathrm{MHz}$ and higher. This had to be taken into account when designing the lowpass filter, to obtain a final $3 \mathrm{~dB}$ cut-off frequency within the specified range of $18 \mathrm{MHz}$ to $20 \mathrm{MHz}$.

The RFM uses two identical but phase shifted ADCs. Both ADCs are fed with the same balanced analogue input signal, which is provided by the analogue input stage. Moreover, the ADCs are operated with a common reference voltage, to improve the balancing between them. The importance of the physical layout was recognized early in the project and capacitors implemented to prevent current loops.

\subsection{The Digital Processing Unit}

The BELA Digital Processing Module (DPM) performs the following tasks:

- Reception of telecommands and their decoding 
Fig. 28 Schematic diagram of the BELA electrical interfaces

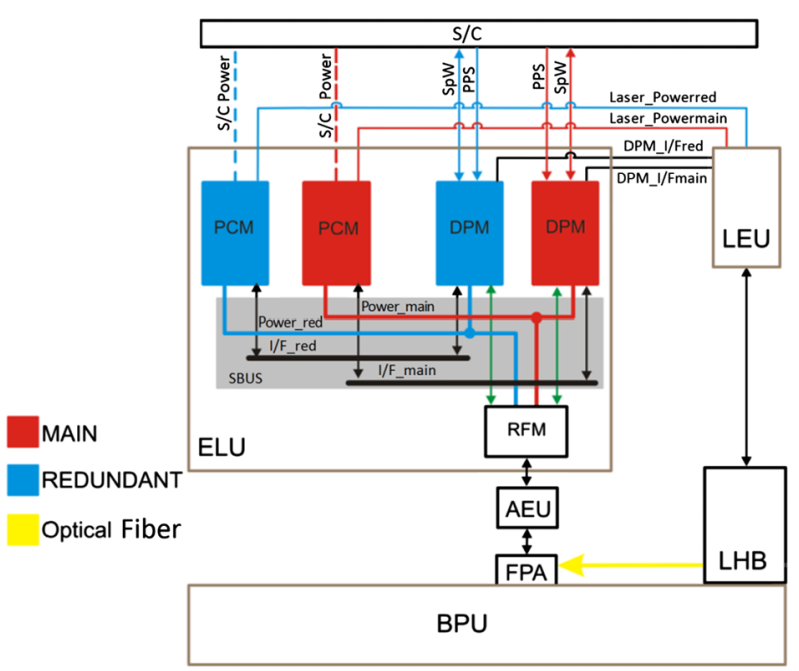

- Laser control, laser pulse initialization

- Control of the RFM operation and setting of ranging parameters (including range tracking)

- Reception and processing of the signal output from the RFM

- Data compression and telemetry formatting and transmission

- Housekeeping data acquisition

- Instrument health check

- Software upload support

Each of the cold-redundant DPMs consists of a single board (Fig. 28). They are included into the Electronics Unit (ELU) which also contains main and redundant Power Converter Module (PCM) and the non-redundant Range Finder Module (RFM).

The communication (telecommand and telemetry) of the DPMs to the spacecraft onboard data handling system is based on the SpaceWire standard ECSS-E-50-12A. Cross strapping between main and redundant configuration between the spacecraft on one side and BELA on the other side is implemented via a router (switching matrix) on the spacecraft side.

The main and redundant DPMs are also served from the spacecraft side with main and redundant PPS timing signals. This connection includes cross-strapping as well. The PPS refers to a time from a reference clock onboard the spacecraft which has an accuracy of better than $10^{-8}$ over 1 day. The scientific data of BELA are based on a time-of-flight measurement of light pulses emitted at a time marked by a stamp which refers to the spacecraft time. Timing errors (ageing and frequency shift) of the RFM master clock are compensated with respect to the PPS signal. In addition, the DPM counts a $1 \mathrm{MHz}$ clock between two PPS signals which allows the time of emission of each shot to be stamped at $1 \mu$ s time resolution. The absolute timing accuracy will depend upon calibrations with the spacecraft system.

The interconnections between the boards inside the ELU are split into 2 separate rigid backplanes:

- A secondary power bus encloses all modules and low-frequency communication interfaces between DPM and PCM. The latter is an LVDS point-to-point interface between each DPM-PCM configuration with drivers and receivers based on UT54LVDS031LV and UT54LVDS032LV. 


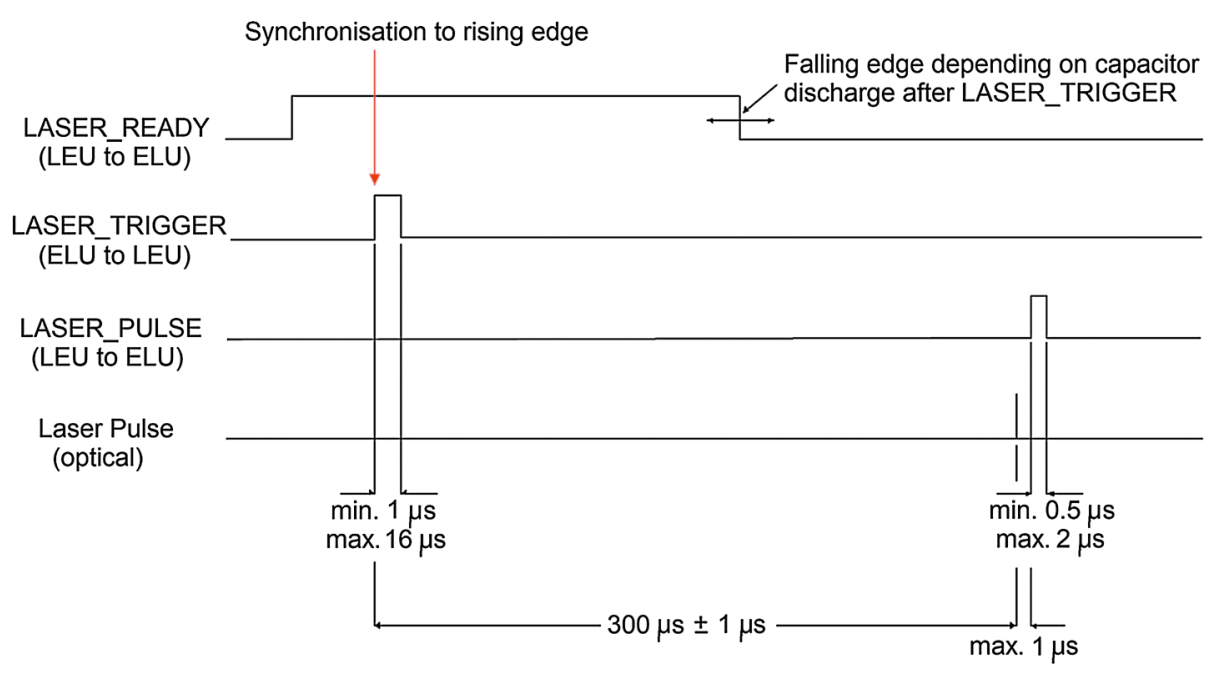

Fig. 29 Digital Processing Module (DPM) - Laser Electronics Unit (LEU) trigger interface

- The implementation of the dedicated DPM-RFM interface accounts for the transition from two DPMs to one non redundant (RFM) structure in terms of power switching and communication. This implementation requires a detection of the active interface (main or redundant) on RFM side.

The logical interface between the ELU/DPM combination and the laser electronics unit (LEU) consists of an asynchronous serial interface with baud-rate of 19200. Communication traffic can be initiated by the DPM only. The laser control module (LCM) command interface inside the LEU uses a tailored version of the Simple Serial Protocol (SSP), designed for master-slave communication on single-master serial buses.

Laser operation is initiated by a simple trigger interface (Fig. 29). The LEU will alert the DPM by a "LASER_READY" such that the capacitor reservoir to drive laser diode pump current is charged. The actual laser pulse emission is then started by a "LASER_TRIGGER". This then triggers the laser diode pump current with a duration of typically $125 \mu$ s to $200 \mu \mathrm{s}$. Then, the laser system will activate the Q-switch in the laser resonator to generate a pulse at $1064 \mathrm{~nm}$ with a typical length of $5 \mathrm{~ns}$. This laser pulse is transmitted towards the planetary surface, while a small fraction is fed to the receiver using optical fibres to provide the start time for the RFM to measure the time of flight. The laser system will then acknowledge with a "LASER_PULSE" signal that an optical pulse has been sent.

The DPM acquires a representative laser energy value during any laser sequence operation. This value is generated by the laser energy monitor. The result of the energy measurement is saved in the DPM and updated during any laser operation sequence. This parameter acts as an initial start value for the next laser operation sequence. The DPM software includes a servo loop code that optimizes laser diode pump time duration and load current of the LEU capacitor reservoir. This regulates the laser pulse energy to a commanded value, nominally $50 \mathrm{~mJ}$. It also avoids the switch-off of the load current of the capacitor reservoir when reaching a maximum voltage. Switching of the load current would result in strong electromagnetic emissions. Therefore, the load current is regulated such that the capacitors are loaded to the optimum voltage to generate a laser pulse with the commanded energy just in the time between two laser shots (nominally $100 \mathrm{~ms}$ ). In this situation, the load current 


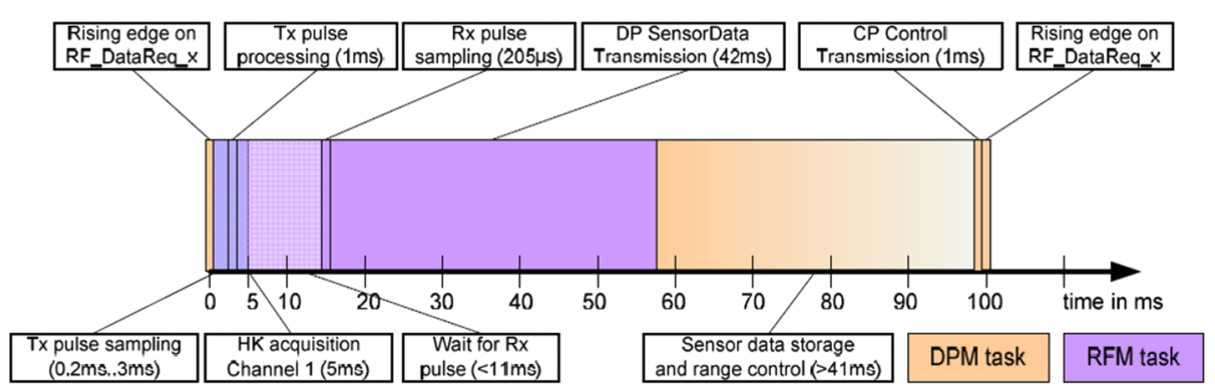

Fig. 30 Timing and tasks in the data mode "Sensor". The system can run a complete cycle in $100 \mathrm{~ms}$. Each task within the cycle is indicated with the time needed to complete that specific operation

has only small temporal changes i.e. it is quasi-steady. The DPM also includes a laser shot counter. The number of counts are saved permanently in the DPM.

The same LASER_TRIGGER signal sent by the DPM to the LEU is also transmitted to the RFM in order to initiate the ranging measurement. The RFM operations are based on triggering used for the cyclic collection of the RFM housekeeping data as well as for the update of the Peltier and HV supply control values. The external trigger signal is used to initiate a RFM processing sequence depending on the data mode.

Two packets are defined for communication between DPM and RFM

a) Control Packets (CP) are sent by the DPM to the RFM. They contain the settings of the complete BELA receiver hardware as well as the settings for the different operation modes.

b) The RFM returns Data Packets (DP) including housekeeping values and science data.

Three receiver science modes have been implemented. The data mode "Sensor" is used to access the complete sensor signal samples (within the Tx or Rx range windows) acquired by the RFM, where the Rx range window sampling (sometimes called pulse sampling) is carried out at a prescribed time delay with respect to the Tx pulse. Other parameters from the CP "Control" used in this data mode are "Rx Gain Code", which selects the gain of the sensor signal path during Rx range window sampling, and "Pulse Repetition Period", which is the time in between two external trigger signals in [msec]. In "Sensor" mode, the time period between two consecutive external trigger signals must be equal to the parameter "Pulse Repetition Period" and at least $100 \mathrm{msec}$, i.e., the highest trigger repetition frequency is $10 \mathrm{~Hz}$.

If the Rx range window (pulse) sampling has finished, the RFM sends the DP "SensorData" to the DPM. Figure 30 summarizes the timing and tasks in the data mode "Sensor" under the condition that a Tx pulse was actually detected in the Tx range window samples during Tx pulse processing.

The data mode "Pulse" is used to process both the Tx and the Rx range window pulses in order to obtain the features of the Tx and the Rx pulse as well as the time (distance) between their detection. The respective timing and tasks executed are presented in Fig. 31.

The data mode "Albedo" is used to access the complete sensor signal samples acquired by the RFM without the detection of a Tx pulse, i.e., the sensor data sampling starts immediately after the reception of the external trigger signal. In the event of laser failure, this mode can be used to recover information about the reflectance of the surface in a passive mode.

An additional "Test" mode is also available but will not be used in flight. 


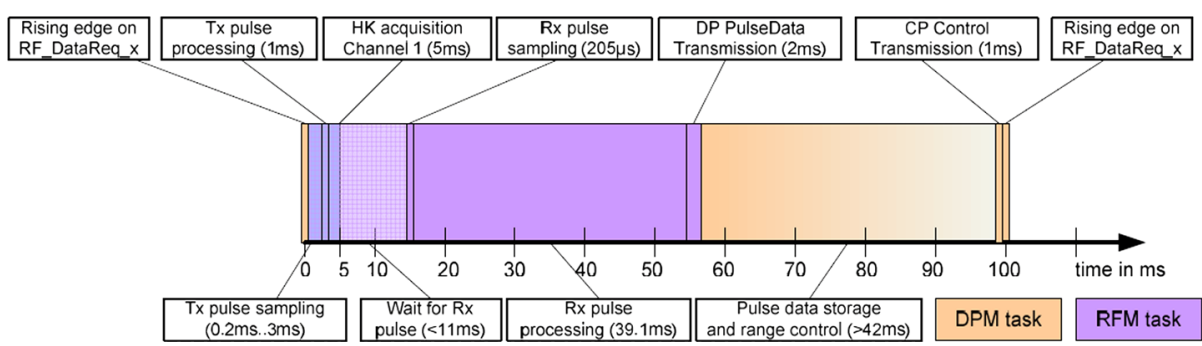

Fig. 31 Timing and Tasking in the data mode "Pulse". The system can run a complete cycle in $100 \mathrm{~ms}$. Each task within the cycle is indicated with the time needed to complete that specific operation. The difference to Fig. 30 is that the samples within the range window are processed before transmission

Table 9 Voltages generated by the PCM. The $28 \mathrm{~V}$ line is taken from the spacecraft and routed through to the laser which has its own voltage regulation

\begin{tabular}{llccc}
\hline Voltage $(\mathrm{V})$ & Tolerance & I min $(\mathrm{mA})$ & I typ $(\mathrm{mA})$ & I max $(\mathrm{mA})$ \\
\hline+3.40 & $\pm 2 \%$ & 1920 & 3950 & 4725 \\
+5.30 & $\pm 2 \%$ & 285 & 575 & 750 \\
-5.30 & $\pm 2 \%$ & 195 & 325 & 365 \\
+12.60 & $\pm 3 \%$ & 0 & 55 & 60 \\
+28 & $-3 \%$ & 0 & 740 & 1000 \\
& $+1 \%$ & & & \\
\hline
\end{tabular}

\subsection{The Power Converter Module (PCM)}

The BELA instrument has been designed to operate with nominal performance within the following steady state voltage limits provided by the spacecraft Power Distribution Unit (PDU) (this is applicable for the main and redundant power lines): Min $=28 \mathrm{~V}-3 \%$, Max $=28 \mathrm{~V}+1 \%$ (see Table 9 ). The instrument will safely survive any standing or fluctuating voltage in the full range 0 to $32 \mathrm{~V}$, and it generates its own secondary power isolated from the input power (Rodrigo et al. 2017).

For those specifications, two identical Power Converter Modules (PCM), main and redundant, are implemented in cold redundant configuration. The PCM performs galvanic isolation from spacecraft primary power and secondary voltage generation. The overall efficiency of the PCM secondary power generation is better than $75 \%$. The PCM limits the inrush current to $1 \mathrm{~A} / \mu \mathrm{s}$. Because of DC/DC galvanic isolated requirements, any power supply pin and the bonding stud of the instrument housings in functional configuration and as well, secondary power lines insulation characteristics from the box structure, must have a capacitance of $<50 \mathrm{nF}$ in parallel with a resistance of $>1 \mathrm{M} \Omega$ when the bonding stud is disconnected from a star point grounding system.

The voltages generated within the PCM are shown in Table 9.

The $+5.3 \mathrm{~V},-5.3 \mathrm{~V}$ and $+12.6 \mathrm{~V}$ outputs are equipped with linear regulators. Due to the high current requirements of the $+3.4 \mathrm{~V}$ output line, this voltage is generated by means of a dedicated buck DC/DC converter in order to avoid the high losses of a linear regulator. The PCM supplies the laser subsystem with the $28 \mathrm{~V}$ spacecraft power bus through a series switch with control of the variability of the current and just after the common mode filter. Figure 32 shows the detailed PCM block diagram which is hereby described.

The final flight model main board is shown in Fig. 33. 


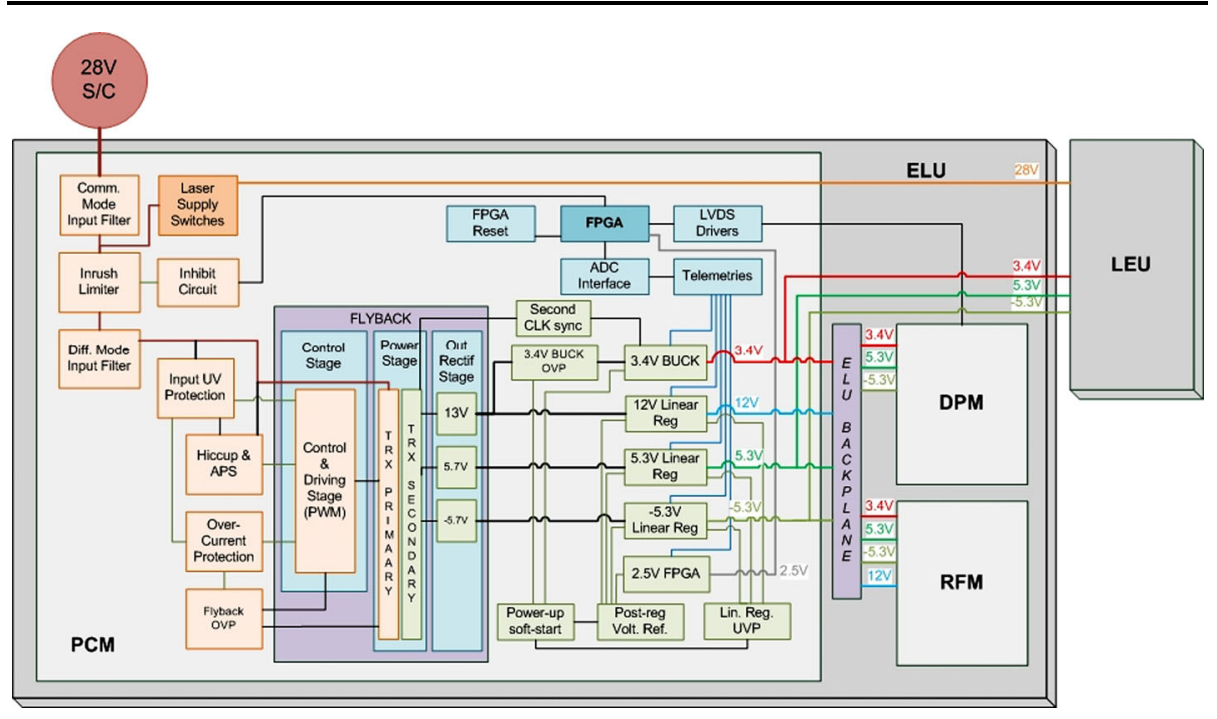

Fig. 32 Power Converter Module (PCM) block diagram

\section{System Integration and Test}

\subsection{Sub-System Integration}

The RTL was integrated onto the baseplate by RUAG Space (now Thales-Alenia Space Switzerland). The FPA incorporating the detector and the BEO was mounted to the back of the RTL to provide an optical receiver chain that could be checked by industry before delivery for higher level integration. The AEU was a separate unit but was delivered simultaneously. RUAG were also responsible for the RFM. They retained a non-flight version of the RFM for testing with the receiver optical chain while delivering the flight version to DLR in Berlin for integration into the ELU.

The ELU was integrated at DLR in Berlin and sub-system level checks of the RFM, DPM, and PCM within the box were satisfactory. This approach was considered to be best because in integrating the receiver section of the instrument with the ELU later in the process, the AEU would merely have to be connected to the RFM in order for the receiver section of the instrument to become operational. In effect the integration here would be simply between elements that had been manufactured by the same Swiss industrial partner. This worked extremely efficiently.

The laser and LEU were manufactured by Cassidian (now Hensoldt), tested by DLR Berlin, and then delivered with the ELU to UBE for system level integration. No electrical integration of the transmitter needed to be performed by UBE, which again simplified the managerial and technical interfaces.

The RBU, the TBU and the SPU were mechanically de-coupled from the rest of the instrument to reduce the conductive thermal load to the instrument and to dump the remaining absorbed heat from the Sun into the spacecraft thermal control system. These units could be delivered directly to the spacecraft with almost no system level testing as they had little influence on the system performance once straylight and thermal balance tests had been completed.

The remaining aspect of the integration was to place the LHB correctly on the baseplate to produce a complete BPU. 

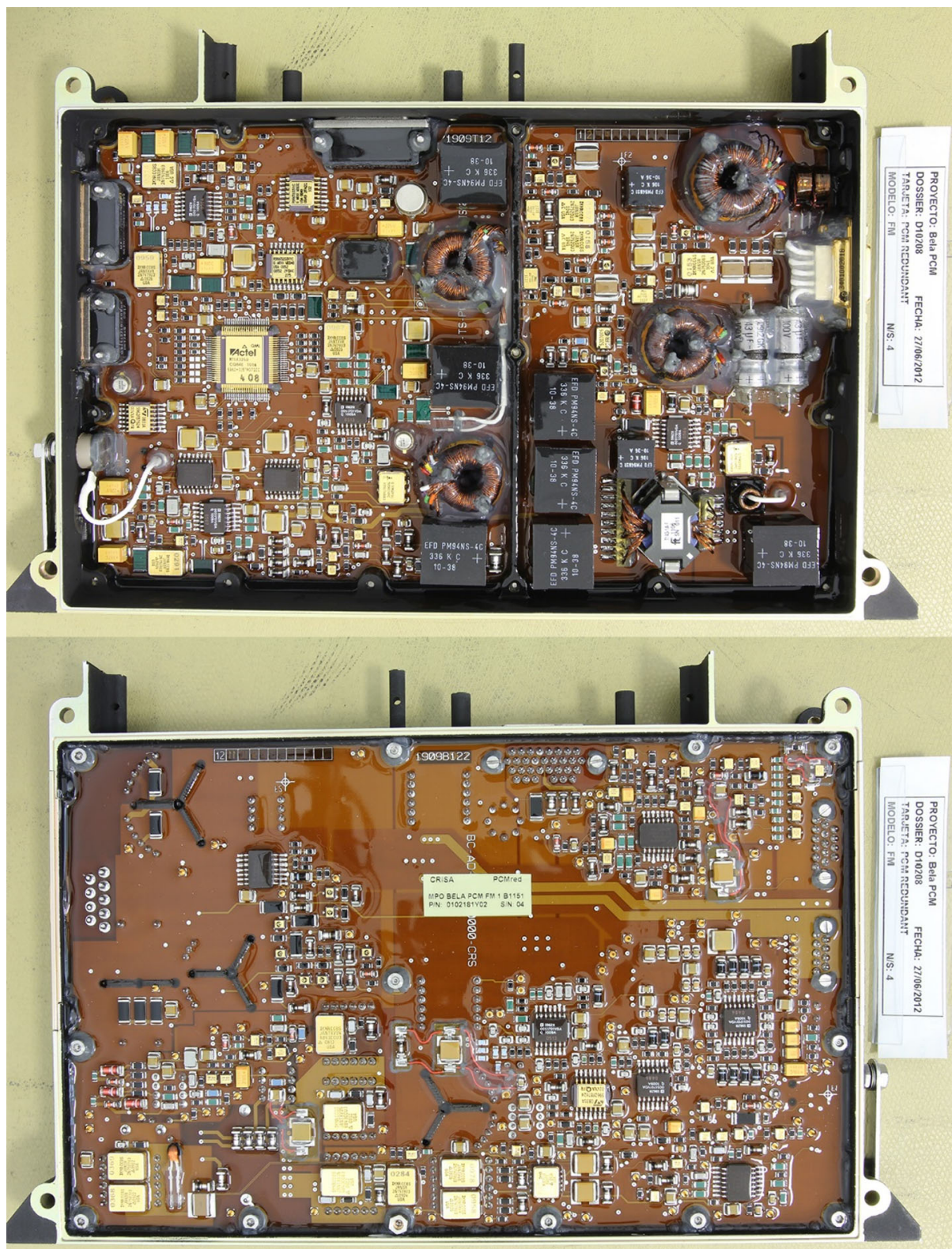

Fig. 33 The Flight Model (FM) Power Converter Module (PCM) board from above and below

\subsection{Alignment}

The transmitter and the receiver were developed in two different countries and then integrated at UBE. A primary concern was the alignment of the transmitter with respect to the receiver and a dedicated facility was built to perform the task. The basic concept is shown in Fig. 34. There were several important aspects. 


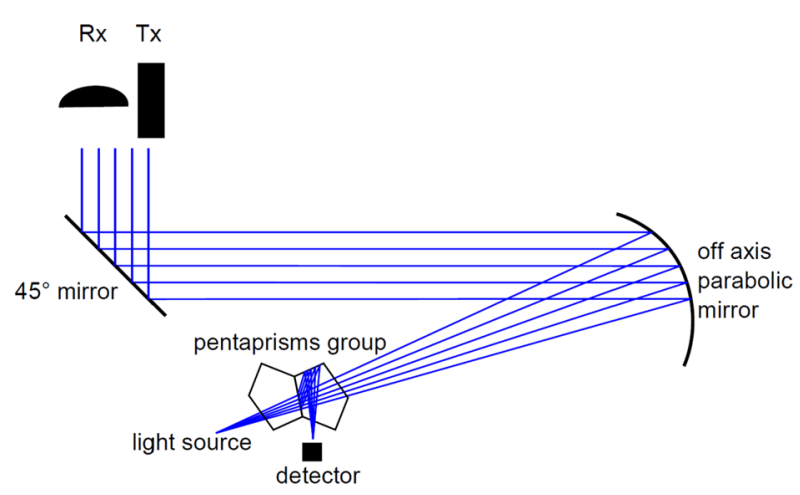

Fig. 34 The basic concept of the alignment facility. The key aspect is the use of the pentaprism group through which light from the transmitter could pass to a detector and through which a light source could pass to the receiver. By using two different wavelengths (the laser wavelength at $1064 \mathrm{~nm}$ and a diode laser at $635 \mathrm{~nm}$ ), coatings on the pentaprism were used to divert the two different wavelengths on different paths allowing alignment without any moving parts or changes in the set-up

The rear of the secondary mirror of the telescope was a plane, gold coated mirror with a cross machined into it (Fig. 13). A diode laser light source $(\lambda=635 \mathrm{~nm})$ at the focus of a large Off-axis Parabolic Mirror (OPM) was used to create a collimated beam. The light was directed towards the BELA laser and telescope, which were mounted on a vertical setup (pointing downwards to reduce distortions in a $1 \mathrm{~g}$ environment). The reflected light from the back of the Secondary Mirror (SM) was aligned to the focus of the OPM. The normal of this mirror with respect to the boresight of the receiver was previously determined by the telescope manufacturer. Hence, the boresight was clearly determined. The laser was then mounted onto the baseplate and emitted pulse was then focused by the OPM to the same effective focal point as the receiver light source by adjusting shims on the laser mounting. An attenuator was needed to reduce the laser power. The detailed procedure was presented by Chakraborty (PhD thesis, Uni Bern, 2012) and in Chakraborty et al. (2012). The sizes of the shims to make the required corrections needed to be computed carefully for the three isostatic mounts.

The receiver field of view was originally required to be $450 \mu \mathrm{rad}$ diameter. The alignment budget (Gouman et al. 2014) took into account residual misalignment and measurement error between transmitter and receiver, the laser divergence, the point ahead angle (resulting from the spacecraft distance of travel during the laser time of flight which could not be pre-compensated because the spacecraft will fly over the planet both "forwards" and "backwards"), and thermal distortion. The image blur resulting from the optics was eventually determined to be the major contributor. This was a consequence of the lightweight structure of the telescope and the difficulty of maintaining the surface form of the primary mirror during machining and coating. Eventually the receiver field of view was slightly increased to ensure $95 \%$ of the signal remained within the field of view at a cost of an increase in reflected background from the planet.

The alignment procedure was verified by a successful open-air test ranging to the mountain, Mönch, from the roof of the University of Bern's Exact Sciences buildings - a distance of just over $60 \mathrm{~km}$ - with the EQM. The misalignment at ambient temperature of the BELA FM instrument was found to be $34 \pm 8 \mu \mathrm{rad}$. 
Fig. 35 Measurements of the position of transmitter footprint within the receiver field of view at different temperatures in vacuo. A systematic trend is visible but the footprint remains well within the receiver field of view (large black circle) over the full temperature range

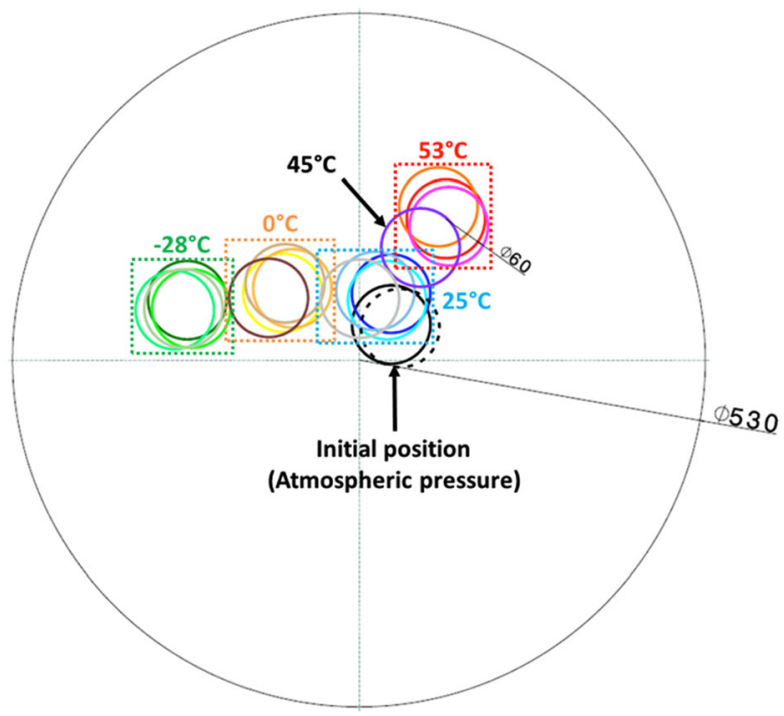

\subsection{Alignment Stability}

BELA is within the spacecraft behind the thermal shield. However, the temperature in orbit will not be constant and will be a strong function of the heliocentric distance. Thermal flexure of the instrument was therefore of major concern and was extensively tested. The test approach was described by Gouman et al. (2014). The testing of the FM instrument was made between $-28{ }^{\circ} \mathrm{C}$ and $+53{ }^{\circ} \mathrm{C}$ and it was demonstrated that the thermal concept and implementation met the requirements.

Figure 35 shows the results of the tests. BELA was placed inside a thermal vacuum chamber. The baseplate orientation, the laser direction and the receiver position were measured from outside the chamber. An optical cube on the baseplate was used as a reference to account for flexure of the chamber itself.

The measurements showed a systematic trend with temperature that had also been seen in the EQM of the instrument. However, the laser beam remained within the field of view of the receiver at all times. The motion of the beam within the field of view defined by the aperture stop combined the APD may have some influence on the final signal to noise of the instrument because of the non-uniformity of the response of the APD when high bias voltages are being applied. This will depend strongly upon the temperature range actually experienced by the instrument in flight and upon the bias voltage chosen as baseline when in orbit.

\subsection{Detector Issues}

A significant issue occurred when the original flight model APD was found to be dead after a system level integration activity. While the root cause of the failure was not proven, several minor changes were made to protect the device better. Firstly, there was a suspicion that the device had been affected by an electrostatic discharge. A possible source was the Ultra High Molecular Weight Polyethylene socket placed behind the detector that was intended to act as additional energetic neutron shielding. This was subsequently removed. Secondly, concern was expressed about operation of the device too close to the breakdown voltage. 
Although a failure from this mechanism could not be reproduced with test devices, it was decided to lower the nominal bias voltage to $340 \mathrm{~V}$ (from $370 \mathrm{~V}$ ) to eliminate any chance of this occurring in flight. The cost here is that the APD gain is reduced from its nominal value of $1.5 \mathrm{MV} / \mathrm{W}$ by around half thereby affecting the PFD. However, the bias voltage can be controlled in software and thus this mitigation measure can be reversed if need be. Thirdly, an additional resistor was added to the circuit to reduce the maximum current through the APD and to the module's High Electron Mobility Transistor gate, during non-nominal illumination events. Finally, a pull down resistor was added in the RFM to ensure the HV set-point stayed below a critical threshold during power up.

It is important to note that there is no indication that the APD module itself was to blame for the failures. Indeed, this device has been demonstrated on numerous occasions to be very robust for this type of application.

\subsection{Electrical Interference Between the Laser and the Receiver}

It was noted relatively late in the programme that the receiver was picking up noise from some other part of the system. This noise was being amplified by the AEU and resulted in a much lower detection threshold than had been predicted. Initially, there was considerable uncertainty about the source because a development model had been placed on the spacecraft for testing and showed no evidence of this noise. However, when the FM was mounted, it became clear that this placeholder model was not sufficiently representative. The problem was ultimately traced to an inadequate specification of the maximum noise level to come from the laser diode driver. The noise entered the grounding loop which, according to spacecraft requirements, joined all sub-systems at a star point. This actually enhanced the problem by producing a conductive path between the laser diode driver and the AEU. The problem was solved by placing Pi-filters (Glenair) at the connectors of the units in the transmitter chain. It was noted that this action also reduced noise on the space wire communications on the spacecraft that had been noted during integrated spacecraft tests. For future implementations, we recommend that the routing and grounding of spacecraft and instrument harnesses should be better defined at an early stage of the project.

\subsection{Ranging Accuracy and Tracking}

Following integration of the BPU, the instrument underwent a series of tests at instrument and spacecraft level. It is important to note that BELA has a series of internal delays resulting from the configuration of the instrument.

The start pulse is passed to the receiver chain using a fibre-optic cable. This leads to the start pulse being detected $2.9 \mathrm{~ns}$ after the pulse has been actually emitted. There is also a delay in the receipt of the return pulse caused by the path length that light travels within the telescope which corresponds to $1.7 \mathrm{~ns}$. Hence there is a net reduction in the distance to the target of $1.2 \mathrm{~ns}$ solely from the instrument configuration when no correction is applied. This value needs to be removed in calibrated data.

Other potential inaccuracies were determined using a set-up with an arbitrary waveform generator (AWG) connected to a modulated laser (Fig. 36). Scenarios could be built using a programmable oscilloscope that triggered the AWG to produce simulated return pulses. The set-up itself needed to be calibrated and was found to have delays of the order of $274 \mathrm{~ns}$. The final measurements indicated that the set-up delays had been well established and that the BELA receiver was determining the time of receipt of the return pulse to an accuracy $<6.67 \mathrm{~ns}$ ( $2 \mathrm{~m}$ in distance and equivalent to its sampling rate) in its coarse detection mode. 


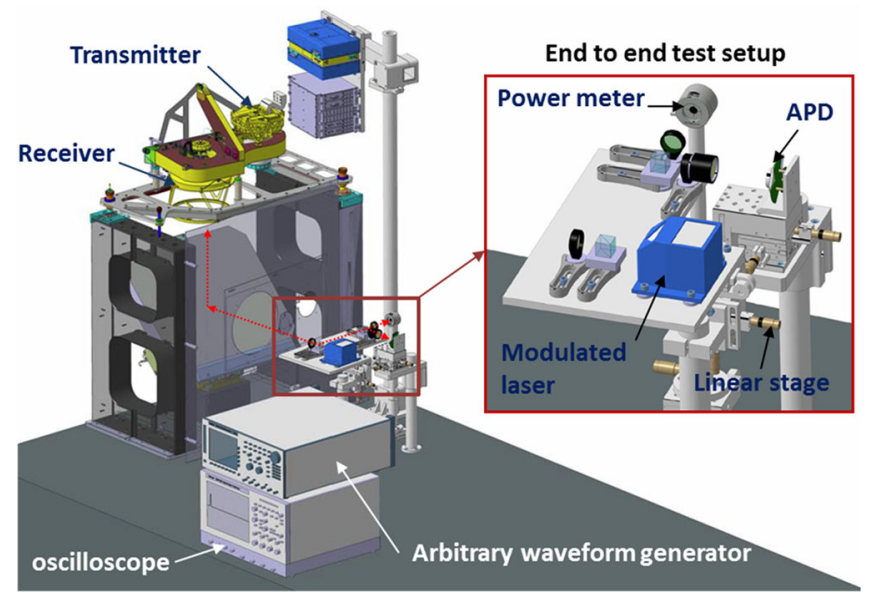

Fig. $36 \mathrm{CAD} / \mathrm{CAM}$ of the set-up used at the University of Bern to support calibration of the BELA instrument

Caution needs to be exercised here with respect to the in-flight calibration because the absolute accuracy also depends upon the characterization of the frequency of the BELA internal oscillator. The instrument counts the number of cycles with respect to the PPS produced by the spacecraft as discussed above and provides this in telemetry. This is intended to calibrate the drift of the internal oscillator. However, this relies upon the spacecraft providing an accurate PPS signal. The PPS frequency may itself drift and thus this needs to be calibrated in-flight by the spacecraft control team in order to reach the desired accuracy.

In its sub-sampling mode, the RFM can provide measurements around a factor of 10 better than the coarse detection mode (under high signal to noise conditions). In the time available, we were not able to verify the absolute accuracy of the flight system at this level. However, relative measurements in sub-sampling mode should be accurate.

A more detailed testing of the qualification model of the RFM was made in a standalone mode to demonstrate the accuracy of the RFM and its algorithm. The RFM was electrically stimulated by simulated laser pulses in order to verify the performance of the subsystem. These pulses were programmed and generated using an arbitrary waveform generator $\left(\right.$ AWG) with a timing resolution of $(1 / 2.7)$ Gsample $\mathrm{s}^{-1}(\approx 0.4 \mathrm{~ns}$ resolution). The procedure to generate a result from one pulse in this test mode are given in Table 10. The sequence could be repeated for an arbitrary number of pulses and the shapes and amplitudes of the pulses could be modified to test various scenarios.

An example of a result is shown in Fig. 37. Taking all results into account (Beck, PhD thesis, Uni Bern, 2011), average height deviations of the order of $5 \mathrm{~cm}$ to $20 \mathrm{~cm}$, depending on the measured pulse width, are evident at high signal to noise. The standard deviations of the measurements are in the same range (around $20 \mathrm{~cm}$ ). For spacecraft heights above $800 \mathrm{~km}$, the measurement accuracy decreases mainly for larger measured pulse widths. It can be stated that generally the time of flight measurement below $1200 \mathrm{~km}$ is very good while the overall average is still in the range of $10 \mathrm{~cm}$ to $30 \mathrm{~cm} \pm 5-40 \mathrm{~cm}$. Above $1200 \mathrm{~km}$ spacecraft height, the measurement accuracy decreases with an average accuracy of $0.5 \mathrm{~m}$ to $1 \mathrm{~m}$ (see e.g. Fig. 37). The RFM also extracts the pulse width and the effective albedo. The pulse width measurement accuracy decreases very quickly for spacecraft heights above $1000 \mathrm{~km}$. An intermediate case is shown in Fig. 38. Consequently, from RFM standalone 
Table 10 Actions occurring during standalone tests to allow time of flight calibration of the Range Finder Module (RFM)

\begin{tabular}{ll}
\hline Step & Action \\
\hline 1 & Control file sent to RFM \\
2 & Trigger signal sent to RFM and AWG \\
3 & Pulse sequence started on AWG; RFM initiates Tx \\
& pulse sampling \\
4 & AWG releases Tx pulse towards RFM \\
5 & RFM Tx pulse processing \\
6 & RFM HK data acquisition \\
7 & RFM wait for range window opening \\
8 & RFM opens range window (Rx pulse sampling) \\
9 & AWG releases Rx pulse \\
10 & RFM Rx pulse processing \\
11 & RFM result data transmission \\
12 & RFM to receive new control file \\
\hline
\end{tabular}

testing, BELA should have an absolute range accuracy of $10-30 \mathrm{~cm}$ in ideal conditions with a small error resulting from unaccounted for delays in the electrical chain between the detector and the RFM input.

HosseiniArani (PhD thesis, Uni Bern, 2020) has indicated that there may also be small systematic errors at the $20 \mathrm{~cm}$ level in the output from the ranging algorithm that could influence measurements attempting to achieve this level of precision.

In addition to the basic accuracy, several other functions were tested at system level.

- The range over which the instrument can successfully range at high signal to noise was tested from $300 \mathrm{~km}$ to $1800 \mathrm{~km}$ successfully.

- An automatic changing of the receiver gain has been implemented in software to increase the signal gain (in the AEU) as the altitude increases. BELA chooses the Rx gain code from a gain look-up table. The gain codes per altitude range can be defined in advance. The automatic switching algorithm was found to work correctly.

- The range tracking algorithm was verified at the nominal maximum rate of change (which can be determined from simple orbital mechanics). This test showed that BELA, operating at $10 \mathrm{~Hz}$, was able to follow an altitude change rate of $420 \mathrm{~m} \mathrm{~s}^{-1}$ from a simulated starting distance of $704.5 \mathrm{~km}$.

- Mercury is well known to have significant scarps resulting from thrust faults. These structures can be $2 \mathrm{~km}$ high. We therefore tested BELA's response to altitude changes at the $2 \mathrm{~km}$ level. In high signal to noise conditions, the system correctly recorded the altitude change.

- BELA contains a scanning algorithm if the expected time of return pulse is completely unknown. This was tested with the default parameters (scanning from $200 \mathrm{~km}$ range to $2000 \mathrm{~km}$ in increments of $\sim 1 / 8$ of the range window per shot). The system was initially set to provide a return pulse at $450 \mathrm{~km}$. This was then switched off and the instrument successfully started to scan. Upon switching on the test pulse again, BELA recovered the pulse correctly. In general, this algorithm would not be used because the spacecraft provides an initial estimate of the distance to the surface so that the time needed to scan can be saved.

- An MLA track over a crater could be implemented in the calibration system by inputting the appropriate delays corresponding to the MLA observation. In this way we could assess 


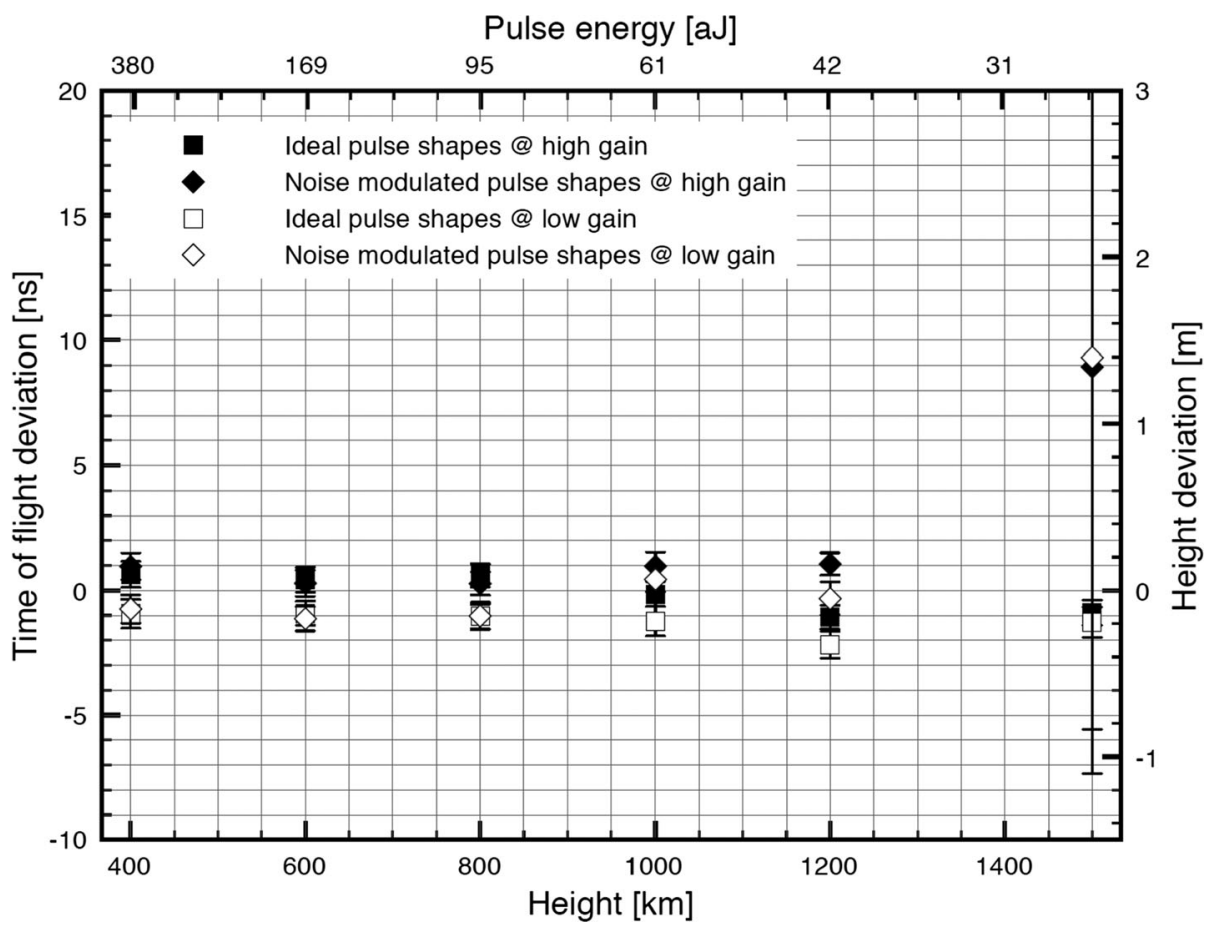

Fig. 37 Range Finder Module (RFM) testing indicating the error in the height measurement as a function of return pulse energy for $20 \mathrm{~ns}$ full-width half maximum pulse shapes

whether BELA could follow a typical Hermean topographic profile as returned by MLA. The instrument successfully performed this on numerous occasions.

- BELA is capable of operating at $5 \mathrm{~Hz}, 2 \mathrm{~Hz}$, and $1 \mathrm{~Hz}$ frequency. The latter is challenging for the tracking algorithm and was studied using the maximum change in elevation resulting from the orbit (see above). This was nonetheless found to work correctly.

The issue with the electrical interference compromised the system level tests for sensitivity and hence a combination of test and analysis was needed. Models of the instrument have been developed over the past 15 years starting with the initial work of Gunderson et al. (2006) and Gunderson and Thomas (2010). The most recently published study was by Steinbrügge et al. (2018) while the BELA team at the University of Bern developed an engineering model of the instrument in software for rapid comparison with test data. As noted by Steinbrügge et al. (2018), calculations are performed in two steps. The first step determines the link budget using the spacecraft altitude and the surface reflectance properties thereby establishing the properties (amplitude, width, time of flight) of the return pulse. The second step establishes the influence of background radiation (reflected sunlight), the effect of the instrument optical characteristics, and the implications of electronics chain (including associated noise).

The noise from the electronics chain for the model calculations was determined by running BELA on the spacecraft and using the output from the instrument in sensor mode. This output was acquired as part of the validation of the noise mitigation measures. Noise samples were obtained both before and after the implementation of the Pi-filters to verify their efficacy. The noise samples after implementation were used to derive the PFD results 


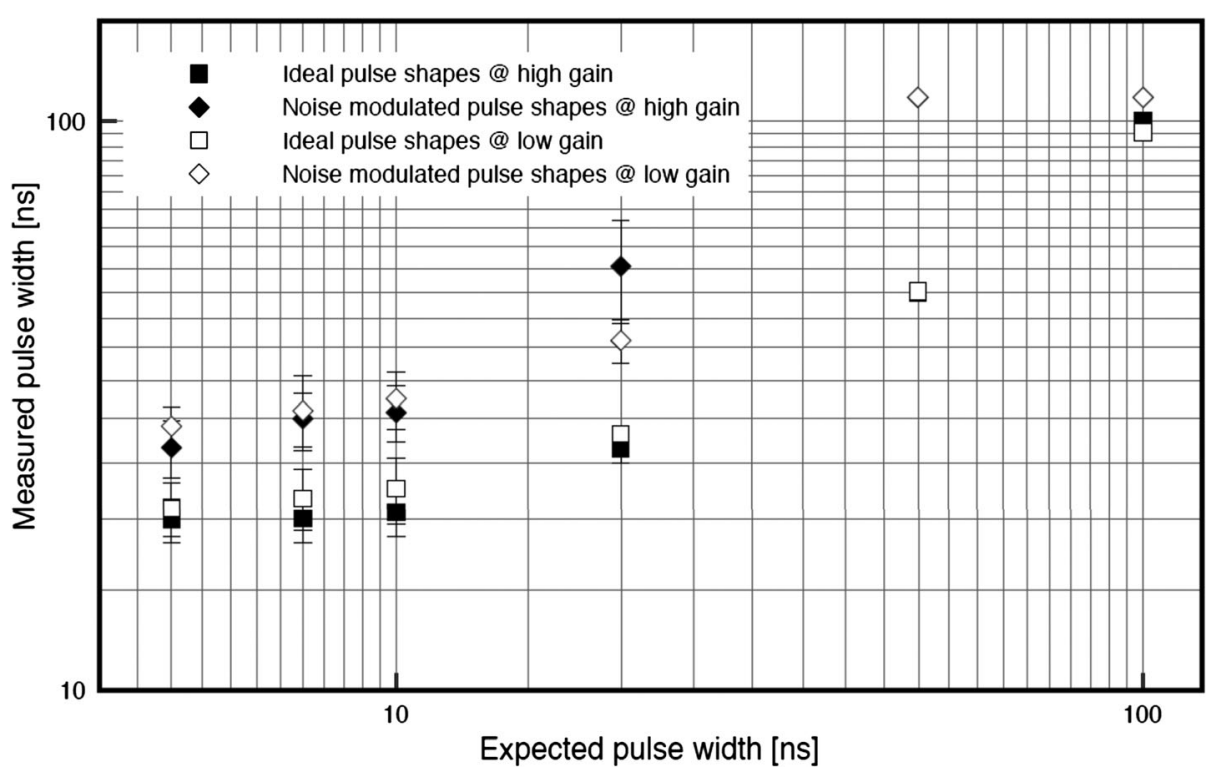

Fig. 38 Range Finder Module (RFM) determination of the pulse width at a simulated altitude of $1200 \mathrm{~km}$. This is a boundary case between accurate pulse width determination at lower altitudes when signal to noise is high, and poor pulse width determination at higher altitudes above the surface

shown in Table 11 and Table 12. These results were obtained using the UBE code but they are consistent to within a few percent with the model provided by Steinbrügge et al. (2018) using a completely independent calculation scheme.

Table 11 shows that as the pulse width broadens, the PFD increases at a specific altitude as might be expected but with the APD bias voltage set to achieve a responsivity of 1.5 $\mathrm{MV} / \mathrm{W}$, the instrument meets its requirement of ranging up to $1055 \mathrm{~km}$. For a return pulse width of $<30 \mathrm{~ns}$, the bandwidth of the electronics dominates the signal width entering the RFM and hence the PFD at a specific altitude is constant for all pulse widths below this.

Table 12 illustrates the effect of using different AEU gain codes and the influence of the bias voltage (and indirectly the APD responsivity). We can see here that the choice of AEU gain code does not have a strong influence on the PFD. However, decreasing the responsivity by a factor of 2 , decreases the maximum working altitude by several hundred kilometres. Consequently, the measures used to protect the APD have a significant effect on the capability of the instrument but the requirement to range to $1055 \mathrm{~km}$ is still met. This can be inverted of course. One could imagine that once the primary objectives of BELA have been met, one could increase the bias voltage to improve the quality of the ranging in a trade-off against risking the APD.

On the basis of the above tables, HosseiniArani et al. (2021) showed that we are able to detect almost all the surface slopes, including the steepest crater walls, from altitudes lower than $670 \mathrm{~km}$, when the APD-A gain = $1.5 \mathrm{MV} / \mathrm{W}$ (and lower than $550 \mathrm{~km}$ when APD-A gain is $0.75 \mathrm{MV} / \mathrm{W}$ ). From higher orbital altitudes, the instrument will only be capable of measuring slopes up to around $15-20^{\circ}$ but this meets the original requirements.

Work has also been performed to assess the influence (worst case) of instrument degradation in flight (resulting from radiation damage, component ageing etc.). There are significant uncertainties but the instrument is well protected and consequently we expect that degradation in the worst case can only lower the working limit of the instrument by around $200 \mathrm{~km}$. 
Table 11 Probability of False Detection (PFD) for an AEU Gain Code $=7$ and an APD responsivity of 1.5 MV/W. Note how the PFD increases as the pulse width broadens. Below $30 \mathrm{~ns}$ pulse width the maximum range is reached at around $1700 \mathrm{~km}$. Yellow boxes indicate marginal PFDs for the instrument. Red boxes indicate critical values with limited detection capability

\begin{tabular}{|c|c|c|c|c|c|c|c|c|c|c|c|c|}
\hline \multirow{2}{*}{$\begin{array}{l}\text { Return } \\
\text { pulse } \\
\text { energy }(\mathrm{J})\end{array}$} & \multirow{2}{*}{$\begin{array}{l}\text { Altitude } \\
(\mathrm{km})\end{array}$} & \multicolumn{11}{|c|}{ FWHM (ns) } \\
\hline & & 10 & 25 & 30 & 35 & 40 & 50 & 60 & 70 & 80 & 90 & 100 \\
\hline $7.93 \mathrm{E}-16$ & 300 & $0 \%$ & $0 \%$ & $0 \%$ & $0 \%$ & $0 \%$ & $0 \%$ & $0 \%$ & $0 \%$ & $0 \%$ & $0 \%$ & $0 \%$ \\
\hline $4.46 \mathrm{E}-16$ & 400 & $0 \%$ & $0 \%$ & $0 \%$ & $0 \%$ & $0 \%$ & $0 \%$ & $0 \%$ & $0 \%$ & $0 \%$ & $0 \%$ & $0 \%$ \\
\hline $2.86 \mathrm{E}-16$ & 500 & $0 \%$ & $0 \%$ & $0 \%$ & $0 \%$ & $0 \%$ & $0 \%$ & $0 \%$ & $0 \%$ & $0 \%$ & $0 \%$ & $0 \%$ \\
\hline $1.98 \mathrm{E}-16$ & 600 & $0 \%$ & $0 \%$ & $0 \%$ & $0 \%$ & $0 \%$ & $0 \%$ & $0 \%$ & $0 \%$ & $0 \%$ & $0 \%$ & $0 \%$ \\
\hline $1.46 \mathrm{E}-16$ & 700 & $0 \%$ & $0 \%$ & $0 \%$ & $0 \%$ & $0 \%$ & $0 \%$ & $0 \%$ & $0 \%$ & $0 \%$ & $0 \%$ & $0 \%$ \\
\hline $1.12 \mathrm{E}-16$ & 800 & $0 \%$ & $0 \%$ & $0 \%$ & $0 \%$ & $0 \%$ & $0 \%$ & $0 \%$ & $0 \%$ & $0 \%$ & $0 \%$ & $0 \%$ \\
\hline $8.81 \mathrm{E}-17$ & 900 & $0 \%$ & $0 \%$ & $0 \%$ & $0 \%$ & $0 \%$ & $0 \%$ & $0 \%$ & $0 \%$ & $0 \%$ & $0 \%$ & $0 \%$ \\
\hline $7.14 \mathrm{E}-17$ & 1000 & $0 \%$ & $0 \%$ & $0 \%$ & $0 \%$ & $0 \%$ & $0 \%$ & $0 \%$ & $0 \%$ & $0 \%$ & $0 \%$ & $0 \%$ \\
\hline $5.90 \mathrm{E}-17$ & 1100 & $0 \%$ & $0 \%$ & $0 \%$ & $0 \%$ & $0 \%$ & $0 \%$ & $0 \%$ & $0 \%$ & $0 \%$ & $0 \%$ & $0 \%$ \\
\hline $4.96 \mathrm{E}-17$ & 1200 & $0 \%$ & $0 \%$ & $0 \%$ & $0 \%$ & $0 \%$ & $0 \%$ & $0 \%$ & $0 \%$ & $2 \%$ & $6 \%$ & $16 \%$ \\
\hline 4.22E-17 & 1300 & $0 \%$ & $0 \%$ & $0 \%$ & $0 \%$ & $0 \%$ & $0 \%$ & $6 \%$ & $8 \%$ & $18 \%$ & $24 \%$ & $52 \%$ \\
\hline $3.64 \mathrm{E}-17$ & 1400 & $0 \%$ & $0 \%$ & $0 \%$ & $2 \%$ & $4 \%$ & $2 \%$ & $20 \%$ & $24 \%$ & $66 \%$ & $72 \%$ & $76 \%$ \\
\hline $3.17 \mathrm{E}-17$ & 1500 & $4 \%$ & $2 \%$ & $2 \%$ & $6 \%$ & $10 \%$ & $20 \%$ & $46 \%$ & $56 \%$ & $66 \%$ & $78 \%$ & $86 \%$ \\
\hline $2.79 \mathrm{E}-17$ & 1600 & $6 \%$ & $8 \%$ & $10 \%$ & $24 \%$ & $38 \%$ & $56 \%$ & $72 \%$ & $74 \%$ & $88 \%$ & $86 \%$ & $94 \%$ \\
\hline $2.47 \mathrm{E}-17$ & 1700 & $30 \%$ & $22 \%$ & $26 \%$ & $40 \%$ & $54 \%$ & $66 \%$ & $74 \%$ & $36 \%$ & $92 \%$ & $96 \%$ & $98 \%$ \\
\hline
\end{tabular}

However, If the return signal is detected the measurement accuracies will not be significantly affected by the degradation effects.

\section{Flight Operations and Data Products}

BELA works optimally when continuously operating in a nadir-pointing geometry from the nominal orbit. This results in a ground-track spacing at the equator of about $25 \mathrm{~km}$. BELA is body-mounted on the MPO so that in order to fill in between ground-tracks, the spacecraft has to roll. This capability appears to be available up to an angle of $5^{\circ}$ off-nadir. This should be sufficient to cover gaps in the surface mapping.

The thermal protection system (the baffles) have been designed assuming that the Sun will not be less than $38^{\circ}$ from the boresight. This value is reached only at apoapsis in the nominal orbit if the instrument is nadir pointing. BELA has been designed thermally to cope with rolling the spacecraft up to $5^{\circ}$ up to this limit. This would allow some flexibility in mapping to prevent large gaps in the coverage and could also be used to force cross-overs at lower latitudes to improve the accuracy of orbit solutions. These aspects will need to be taken into account in the final operations planning.

\subsection{BELA Data Products}

BELA data allow the determination of the distance to the surface from the time of flight of detected laser return pulses, while the intensity and pulse width provide information on the albedo of the surface and on its slopes and roughness, respectively. Here we summarize the characteristics of the BELA raw and calibrated data products to be released through 
Table 12 The probability of False Detection (PFD) determined using different AEU gain codes. A comparison between using the APD at its highest responsivity (1.5 MV/W) and the lower (possibly safer) value of $0.75 \mathrm{MV} / \mathrm{W}$ is also made. The instrument meets its fundamental requirement of ranging to $1055 \mathrm{~km}$ in all cases. A FWHM of the return pulse of $35 \mathrm{~ns}$ corresponds to roughly $4.2 \mathrm{deg}$ of slope at $1000 \mathrm{~km}$

\begin{tabular}{|c|c|c|c|c|c|c|c|}
\hline \multicolumn{8}{|c|}{$\mathrm{FWHM}=35 \mathrm{~ns}$} \\
\hline \multirow{3}{*}{$\begin{array}{l}\text { Distance } \\
(\mathrm{km})\end{array}$} & \multirow{3}{*}{$\begin{array}{l}\text { Pulse } \\
\text { energy }(J)\end{array}$} & \multicolumn{6}{|l|}{ PFD } \\
\hline & & \multicolumn{2}{|l|}{ Gain code 0} & \multicolumn{2}{|l|}{ Gain code 7} & \multicolumn{2}{|c|}{ Gain code 15} \\
\hline & & $\begin{array}{l}\text { APD resp. } \\
=1.5\end{array}$ & $\begin{array}{l}\text { APD resp } \\
=0.75\end{array}$ & $\begin{array}{l}\text { APD resp } \\
=1.5\end{array}$ & $\begin{array}{l}\text { APD resp } \\
=0.75\end{array}$ & $\begin{array}{l}\text { APD resp } \\
=1.5\end{array}$ & $\begin{array}{l}\text { APD resp } \\
=0.75\end{array}$ \\
\hline 300 & $7.93 \mathrm{E}-16$ & $0 \%$ & $0 \%$ & $0 \%$ & $0 \%$ & $0 \%$ & $0 \%$ \\
\hline 400 & $4.46 \mathrm{E}-16$ & $0 \%$ & $0 \%$ & $0 \%$ & $0 \%$ & $0 \%$ & $0 \%$ \\
\hline 500 & $2.86 \mathrm{E}-16$ & $0 \%$ & $0 \%$ & $0 \%$ & $0 \%$ & $0 \%$ & $0 \%$ \\
\hline 600 & $1.98 \mathrm{E}-16$ & $0 \%$ & $0 \%$ & $0 \%$ & $0 \%$ & $0 \%$ & $0 \%$ \\
\hline 700 & $1.46 \mathrm{E}-16$ & $0 \%$ & $0 \%$ & $0 \%$ & $0 \%$ & $0 \%$ & $0 \%$ \\
\hline 800 & $1.12 \mathrm{E}-16$ & $0 \%$ & $0 \%$ & $0 \%$ & $0 \%$ & $0 \%$ & $0 \%$ \\
\hline 900 & $8.81 \mathrm{E}-17$ & $0 \%$ & $0 \%$ & $0 \%$ & $0 \%$ & $0 \%$ & $0 \%$ \\
\hline 1000 & $7.14 \mathrm{E}-17$ & $0 \%$ & $20 \%$ & $0 \%$ & $2 \%$ & $0 \%$ & $2 \%$ \\
\hline 1100 & $5.90 \mathrm{E}-17$ & $0 \%$ & $44 \%$ & $0 \%$ & $14 \%$ & $0 \%$ & $16 \%$ \\
\hline 1200 & $4.96 \mathrm{E}-17$ & $0 \%$ & $70 \%$ & $0 \%$ & $30 \%$ & $0 \%$ & $48 \%$ \\
\hline 1300 & $4.22 \mathrm{E}-17$ & $2 \%$ & $80 \%$ & $0 \%$ & $70 \%$ & $0 \%$ & $66 \%$ \\
\hline 1400 & $3.64 \mathrm{E}-17$ & $4 \%$ & $86 \%$ & $2 \%$ & $82 \%$ & $0 \%$ & $86 \%$ \\
\hline 1500 & $3.17 \mathrm{E}-17$ & $32 \%$ & $94 \%$ & $6 \%$ & $96 \%$ & $0 \%$ & $96 \%$ \\
\hline 1600 & $2.79 \mathrm{E}-17$ & $40 \%$ & $94 \%$ & $24 \%$ & $96 \%$ & $20 \%$ & $96 \%$ \\
\hline 1700 & $2.47 \mathrm{E}-17$ & $82 \%$ & $100 \%$ & $40 \%$ & $98 \%$ & $42 \%$ & $100 \%$ \\
\hline
\end{tabular}

the Planetary Science Archive (PSA) at regular intervals. We also discuss BELA derived data products, which will rely on scientific data processing, analysis of external data, and aggregated BELA datasets. As one of the primary derived properties, absolute position with respect to a body-fixed coordinate system allows assessment of topographic heights, and studies of the global shape properties and planetary rotational dynamics. Precise BELA point measurements will be used to create digital terrain models that can serve as global geodetic reference datasets for Mercury. The topography maps as well as other global maps such as surface roughness maps will also be used for quantitative investigation of Hermean geology, such as surface morphology, tectonics, volcanism, surface ages, and the evolution of the planet.

BELA generates the following observational data for its three different regular operation modes providing science data:

1) In RFM PULSE mode, the pulse discrimination operates on both emitted and received pulse, and a set of pulse detection parameters (centroid position and FWHM) is generated for the emitted pulse and for up to four candidates for received pulse. Thereby the four candidates comprise the most powerful signals within the range window (RW). The range window is a time interval of $200.8 \mu$ s at which the instrument expects the arrival of the return pulse. Optionally, correlation window (CW) samples (CWS), i.e. sub-sets of the original, time-resolved range window samples (RWS), can be recorded.

2) In RFM SENSOR mode, pulse discrimination operates on the outgoing laser pulse only, while the complete set of range window sample (RWS) values is recorded for the incoming signal. 
Table 13 BELA operation modes providing science data and associated product data

\begin{tabular}{|c|c|c|c|c|}
\hline $\begin{array}{l}\text { Operation } \\
\text { mode }\end{array}$ & $\begin{array}{l}\text { Types of data items } \\
\text { for emitted signal }\end{array}$ & $\begin{array}{l}\text { Types of data items } \\
\text { for received signal }\end{array}$ & $\begin{array}{l}\text { Product } \\
\text { files }^{\mathrm{a}}\end{array}$ & Short description \\
\hline PULSE & $\begin{array}{l}\text { - parameters for timing, } \\
\text { intensity, and pulse width } \\
\text { - up to } 42 \mathrm{CW} \text { sensor } \\
\text { samples }\end{array}$ & $\begin{array}{l}\text { - parameters for timing, } \\
\text { intensity, and pulse width } \\
\text { - for up to } 4 \text { pulse } \\
\text { candidates } \\
\text { - up to } 4 \times 42 \mathrm{CW} \text { sensor } \\
\text { samples }\end{array}$ & $\begin{array}{l}\text { ADR } \\
\text { CWS }\end{array}$ & $\begin{array}{l}\text { A data-efficient ranging } \\
\text { mode providing processed } \\
\text { pulse parameters, i.e. } \\
\text { on-board analysis is } \\
\text { applied on emitted and } \\
\text { received pulse. No or } \\
\text { limited number of CW } \\
\text { sensor samples } \\
\text { transmitted. }\end{array}$ \\
\hline SENSOR & $\begin{array}{l}\text { - timing, intensity, pulse } \\
\text { width } \\
\text { - up to } 42 \mathrm{CW} \text { sensor } \\
\text { samples }\end{array}$ & $\begin{array}{l}\text { - } 16064 \text { RW sensor } \\
\text { samples }\end{array}$ & $\begin{array}{l}\text { ADR } \\
\text { CWS } \\
\text { RWS }\end{array}$ & $\begin{array}{l}\text { A ranging mode providing } \\
\text { the complete set of range } \\
\text { window samples, i.e. } \\
\text { on-board analysis is } \\
\text { applied to the emitted } \\
\text { pulse only, the received } \\
\text { signal is recorded by the } \\
\text { full set of RW sensor } \\
\text { samples. }\end{array}$ \\
\hline ALBEDO & no laser shot emitted & - 16064 sensor samples & $\begin{array}{l}\text { ADR } \\
\text { RWS }\end{array}$ & $\begin{array}{l}\text { Passive measurement of } \\
\text { the optical signal at the } \\
\text { laser wavelength of } \\
1064 \mathrm{~nm} \text {, recorded by the } \\
\text { full set of sensor samples. }\end{array}$ \\
\hline
\end{tabular}

${ }^{\mathrm{a}} \mathrm{ADR}=$ Altimetry Data Records, CWS $=$ Correlation Window Samples, RWS $=$ Range Window Samples

3) In RFM ALBEDO mode, the instrument passively measures the ambient radiation at the laser wavelength of $1064 \mathrm{~nm}$ and records a full set of sensor sample values, for the time equivalent of one range window. The data records in BELA data product files resulting from each of these operation modes are denoted as PULSE data records, SENSOR data records, and ALBEDO data records, respectively.

Table 13 provides an overview of the main product data contents for the three regular BELA operation modes providing science data. Note that the selection of data items included in the science telemetry is configurable by software, according to data volume constraints. The selection is controlled by data masks which can be modified by telecommand, or amended by new ones. The data masks are used to control the telemetry data rate and may e.g. differ by the number of transmitted return pulse candidates. Different data masks will be used dependent on the signal-to-noise ratio, which is mainly driven by the ranging distance. With the usage of different data masks, a large variability in the content of the received data packets from BELA is obtained. In contrast to this, the archival BELA data products are adopting a fixed, more user-friendly set of data structures, which, because of the large potential variability of BELA telemetry content, implies that certain subsets of data items or fields might be unused in a given product file (i.e. contain Null values).

BELA raw data products store BELA measurement data in a form close to the telemetry data received from the spacecraft, in accordance with the current PDS4 standard (JPL 2019). They include all of the BELA telemetry data items needed to produce the next higher product level (Calibrated), subsequently. Units are not converted, no ancillary calibration data and no data resampling is applied. Reformatting of data items to obtain standard data 


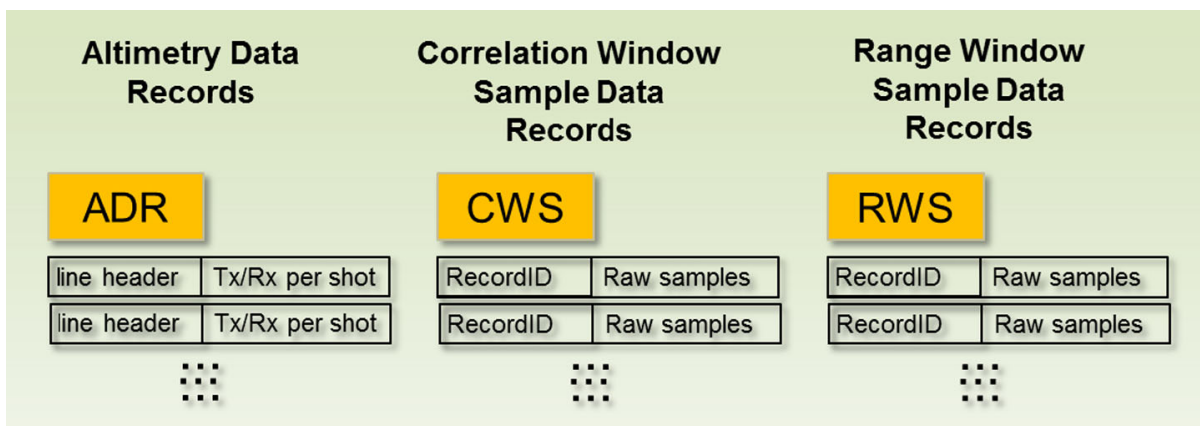

Fig. 39 File organization for BELA Raw data tables

types is applied where telemetry data items are packed into customary bit representations or compound data records to reduce the telemetry data volume.

BELA raw data are stored in binary data tables (called Altimetry Data Records, ADR), each containing data from one Mercury orbit. Two types of ancillary binary data tables containing collections of sensor sample data records are provided optionally, depending on the instrument operation mode. Correlation window sample tables (CWS), i.e. $0.525 \mu$ s subsets of the range window containing a return pulse candidate, are produced when PULSE or SENSOR data records are included in the telemetry data. Range window sample tables (RWS), containing the complete $200.8 \mu$ s range window time interval, are produced when SENSOR or ALBEDO data records are included in the telemetry data. The general structure of the three types of data tables is the same for all operation modes (see Fig. 39 for a schematic representation). All tables are fixed-length binary tables and are organized on a "per shot" basis, i.e. data related to one laser shot (or reflectance measurement in ALBEDO mode) are reported on a single row. Each set of sensor sample values in the optional ancillary sample tables is linked to a unique data record (i.e. row) in the ADR data table.

Each row in the Raw ADR tables basically consists of a line header followed by a set of data items recording the observation data for emitted pulse signals (Tx signal) and received signals (Rx signal). In PULSE mode data records, Rx parameters for up to four alternative return pulse candidates can be present. The line header provides identifiers for the type of data record and index numbers showing whether corresponding data records in the CWS and RWS tables are present and, if applicable, their row number. In addition, the line header contains timing and gain parameters for the data record. The rows of CWS and RWS tables consist of a record identifier storing the row index of the respective data record in the ADR table, followed by the sensor sample values.

BELA Calibrated data products store BELA measurement data in number and time stamp formats, in accordance with the current PDS4 standard (JPL 2019). Data values are converted to physical units, where applicable. The timing of BELA observations is controlled by a comprehensive set of different clock counts and time stamps contained in the telemetry data, which are reduced to unique observation times in the calibrated product.

While the general structure of BELA Calibrated data tables is the same as for Raw data tables (Fig. 39), they partly differ in terms of contained data items. The Calibrated product, for example, includes two preliminary ranging parameters in PULSE mode data records: the NominalTimeOfFlight, providing the measured time difference in milliseconds between emitted and return pulse, according to the nominal value for the sampling rate, and the CoarseRange, providing the measured distance to the surface along the line of sight in kilo- 
meters, according to NominalTimeOfFlight, and not considering the relative motion between s/c and target. As for the Raw product, Rx parameters and ranging parameters for up to four alternative return pulse candidates can be present. BELA Calibrated CWS and RWS tables, if present, contain the sensor samples available also in the corresponding table of the Raw product, now corrected for electronic offsets and normalized according to the gain factor of the analog electronics.

BELA derived data products will rely on scientific processing methodology and will require, as input for their production, external observation data (e.g. spacecraft position and attitude)and/or aggregated BELA data sets, the latter consisting of data from multiple orbital passes for a region, or globally. Three categories of BELA Derived products can be distinguished, based on the type and coverage (spatial and temporal) of required input data:

1) Calibrated geo-located altimetry data records (i.e. 3D surface points), and derived height profiles, 3D models, and map products, will result from the combination of BELA calibrated data with data on spacecraft position and attitude. This allows converting from inertial to body-fixed co-ordinates and applying two-way light-time correction including the effect of relative motion between spacecraft and body.

2) Results from analysis of an aggregated and, ultimately, global BELA data set. In addition to an increased resolution of regional data products, an example for additional information obtainable from aggregated datasets are profile offsets determined at crossoverpoints.

3) Results from joint analysis of BELA data with observational data from other scientific instruments.

Derived data products are expected to undergo revision during the course of the mission, as the knowledge on instrument geometry, spacecraft position, spacecraft attitude, and planetary rotational dynamics may evolve, and while additional observational data become available.

\section{Concluding Remarks}

The BELA instrument is the first European laser altimeter built for interplanetary flight. It was successfully delivered to the spacecraft and, following launch in October 2018, it has been commissioned successfully. The design and implementation evolved during the development phase while the scientific and instrument requirements have been fully met and verified in end-to-end testing on ground. Unlike previous laser altimeters flown in planetary applications, BELA uses a digital approach for range detection and pulse analysis. The ranging accuracy is expected to be better than 2 metres in most cases with values of $20 \mathrm{~cm}$ possible under optimum conditions from the given, slightly elliptical, orbit. Hence, BELA should return a consistent data set for the most if not all of the planet. Over the first 176 days BELA will map the surface to provide a first global topography map even if only one hemisphere can be mapped per orbit. This will continuously be improved by filling the gaps on subsequent orbits. Its $10 \mathrm{~Hz}$ shot frequency, will provide accurate along-track characterization of geological features, e.g. crater structures, ejecta properties and scarps, over the whole planet. The polar orbit is ideal for investigation of the unilluminated interiors of craters at both north and south polar regions that may conceal volatiles including water ice. Crosscorrelation with MLA data in the northern hemisphere should provide additional constraints that warrant detailed investigation. 
Acknowledgements This work was supported by the Swiss State Secretariat for Education, Research and Innovation (SERI) under the PRODEX scheme (contract number C90191). The opinions expressed and arguments employed herein do not necessarily reflect the official views of the Swiss Government. DLR would like to thank all involved collaborators in the BELA transmitter team. Financial support was provided by the DLR Space Administration on behalf of the Federal Ministry of Economics and Technology of Germany. We acknowledge the financial support from the State Agency for Research of the Spanish MCIU through the "Center of Excellence Severo Ochoa" award SEV-2017-0709, and from the research projects ESP 201454062-R, ESP 2016-76076-R and PGC 2018-099425-B-I00.

The authors would especially like to thank A. Himmes, J. Frauchiger, A. Werthmueller, F. Laforce, A. Pandy, B. Lüthi, K. Kudielka, M. Coradini, B. Gramkow, J. Louet, U. Reininghaus, C. Scharmberg, M. Schwetterle, P. Jensen, M. Lazerges, J. van Casteren, K. Seiferlin, S. Graf,J. Benkhoff, J. Zender, E. Montagnon, A. Dietz, F. Giannini, U. Heer, and the BepiColombo science and operations teams at ESTEC, ESOC, and ESAC for their support.

The authors would also like to thank two anonymous referees for comments and suggestions that significantly improved the manuscript.

Funding Note Open Access funding provided by Universität Bern.

Publisher's Note Springer Nature remains neutral with regard to jurisdictional claims in published maps and institutional affiliations.

Open Access This article is licensed under a Creative Commons Attribution 4.0 International License, which permits use, sharing, adaptation, distribution and reproduction in any medium or format, as long as you give appropriate credit to the original author(s) and the source, provide a link to the Creative Commons licence, and indicate if changes were made. The images or other third party material in this article are included in the article's Creative Commons licence, unless indicated otherwise in a credit line to the material. If material is not included in the article's Creative Commons licence and your intended use is not permitted by statutory regulation or exceeds the permitted use, you will need to obtain permission directly from the copyright holder. To view a copy of this licence, visit http://creativecommons.org/licenses/by/4.0/.

\section{References}

J.B. Abshire, J.C. Smith, B.E. Schutz, The Geoscience Laser Altimeter System (GLAS), Space Technology and Applications. AIP Conference Proceedings, vol. 420 (1998), p. 33

J.B. Abshire, X. Sun, R.S. Afzal, Mars orbiter laser altimeter: receiver model and performance analysis. Appl. Opt. 39, 2449 (2000)

J.B. Abshire, X. Sun, H. Riris, M. Sirota, J. McGarry, S. Palm, D. Yi, P. Liiva, Geoscience Laser Altimeter System (GLAS) on the ICESat mission: on-orbit measurement performance. Geophys. Res. Lett. 21, L21S02 (2005). https://doi.org/10.1029/2005GL024028

C. Althaus, H. Michaelis, H. Hussmann, K. Lingenauber, R. Kallenbach, S. Del Togno, F. Lüdicke, BELA transmitter performance and pointing stability verification campaign at DLR-PF. Acta Astronaut. 154, 103-118 (2019)

H. Araki, S. Tazawa, H. Noda, Y. Ishihara, S. Goossens, S. Sasaki, N. Kawano, I. Kamiya, H. Otake, J. Oberst, C. Shum, Lunar global shape and polar topography derived from Kaguya-LALT laser altimetry. Science 323, 897-900 (2009)

H. Becht, H. Hubach, M. Rech, M. Weispfennig, Efficient lasers for remote sensing. Proc. SPIE 7686, 76860D (2010)

T. Beck, B.S. Lüthi, G. Messina, D. Piazza, K. Seiferlin, N. Thomas, Thermal analysis of a reflective baffle designed for space applications. Acta Astronaut. 69, 323 (2011)

T. Behnke, M. Fuji, R. Kallenbach, M. Kobayashi, R. Henkelmann, Y. Sato, J. Uhrle, Laser ranging signal detection in harsh EMC environment, in ESA Workshop on Aerospace EMC, Budapest, Hungary, 20-22 May 2019 (IEEE Press, New York, 2019). https://doi.org/10.23919/AeroEMC.2019.8788946

Benkhoff et al., Space Sci. Rev. (2021). this journal

Y. Berk, Y. Karni, G. Klumel, M. Levy, Y. Openhaim, S. Risemberg, M. Rech, H. Becht, B. Frei, F. Monti di Sopra, Space-grade reliability of $808 \mathrm{~nm}$ QCW laser diode arrays (LDAs) delivering over 20 billion shots, in High-Power Diode Laser Technology and Applications VII, vol. 7198 (2009), $71980 \mathrm{C}$

J.B. Blair, D.L. Rabine, M.A. Hofton, The Laser Vegetation Imaging Sensor: a medium-altitude, digitisationonly, airborne laser altimeter for mapping vegetation and topography. ISPRS J. Photogramm. Remote Sens. 54, 115 (1999) 
J. Blazej, I. Prochazka, K. Hamal, M. Fedyszynova, F. Yang, P. Huang, H. Michaelis, U. Schreiber, Photon counting laser altimeter for planetary exploration - the technology demonstrator. J. Opt. A, Pure Appl. Opt. 9, S98 (2007)

J.L. Bufton, Laser altimetry measurements from aircraft and spacecraft. Proc. IEEE 77, 463 (1989)

J. Bufton, B. Blair, J. Cavanaugh, J. Garvin, Shuttle Laser Altimeter (SLA): a pathfinder for space-based laser altimetry and lidar, in The 1995 Shuttle Small Payloads Symposium (1995), pp. 83-90 (SEE N96-13754 02-12, 83)

J.F. Cavanaugh, J.C. Smith, X. Sun, A.E. Bartels, L. Ramos-Izquierdo, D.J. Krebs, J.F. McGarry, R. Trunzo, A.M. Novo-Gradac, J.L. Britt, J. Karsh, R.B. Katz, A.T. Lukemire, R. Szymkiewicz, D.L. Berry, J.P. Swinski, G.A. Neumann, M.T. Zuber, D.E. Smith, The Mercury laser altimeter instrument for the MESSENGER mission. Space Sci. Rev. 131, 451 (2007)

S. Chakraborty, M. Affolter, K. Gunderson, J. Neubert, N. Thomas, T. Beck, M. Gerber, S. Graf, D. Piazza, A. Pommerol, G. Roethlisberger, K. Seiferlin, High accuracy alignment facility for the receiver and transmitter of the BepiColombo Laser Altimeter. Appl. Opt. 51, 4907 (2012)

E.R. Cohen, B.N. Taylor, The fundamental physical constants. Phys. Today 50, BG7 (1997)

T.D. Cole, M.T. Boies, A.S. El-Dinary, A. Cheng, M.T. Zuber, D.E. Smith, The near-Earth asteroid rendezvous laser altimeter. Space Sci. Rev. 82, 217 (1997)

G. Cremonese, F. Capaccioni, M.T. Capria, A. Doressoundiram, P. Palumbo, M. Vincendon, M. Massironi, S. Debei, M. Zusi, F. Altieri, M. Amoroso, G. Aroldi, M. Baroni, A. Barucci, G. Bellucci, J. Benkhoff, S. Besse, C. Bettanini, M. Blecka, D. Borrelli, J.R. Brucato, C. Carli, V. Carlier, P. Cerroni, A. Cicchetti, L. Colangeli, M. Dami, V. Da Deppo, V. Della Corte, M.C. De Sanctis, S. Erard, F. Esposito, D. Fantinel, L. Ferranti, F. Ferri, I. Ficai Veltroni, G. Filacchione, E. Flamini, G. Forlani, S. Fornasier, O. Forni, M. Fulchignoni, V. Galluzzi, K. Gwinner, W. Ip, L. Jorda, Y. Langevin, L. Lara, F. Leblanc, C. Leyrat, Y. Li, S. Marchi, L. Marinangeli, F. Marzari, E. Mazzotta Epifani, M. Mendillo, V. Mennella, R. Mugnuolo, K. Muinonen, G. Naletto, R. Noschese, E. Palomba, R. Paolinetti, D. Perna, G. Piccioni, R. Politi, F. Poulet, R. Ragazzoni, C. Re, M. Rossi, A. Rotundi, G. Salemi, M. Sgavetti, E. Simioni, N. Thomas, L. Tommasi, A. Turella, T. Van Hoolst, L. Wilson, F. Zambon, A. Aboudan, O. Barraud, N. Bott, P. Borin, G. Colombatti, M. El Yazidi, S. Ferrari, J. Flahaut, L. Giacomini, L. Guzzetta, A. Lucchetti, E. Martellato, M. Pajola, A. Slemer, G. Tognon, D. Turrini, SIMBIO-SYS: Scientific cameras and spectrometer for the BepiColombo mission. Space Sci. Rev. 216(5), 75 (2020). https://doi.org/10.1007/s11214-020-00704-8. http://adsabs.harvard.edu/abs/2020SSRv..216...75C

M.G. Daly, O.S. Barnouin, C. Dickinson, J. Seabrook, C.L. Johnson, G. Cunningham, T. Haltigin, D. Gaudreau, C. Brunet, I. Aslam, A. Taylor, E.B. Bierhaus, W. Boynton, M. Nolan, D.S. Lauretta, The OSIRISREx Laser Altimeter (OLA) investigation and instrument. Space Sci. Rev. 212(1), 899-924 (2017)

C.S. Gardner, Ranging performance of satellite laser altimeters. IEEE Trans. Geosci. Remote Sens. 30, 1061 (1992)

Genova et al., Geodesy, geophysics and fundamental physics investigations of the BepiColombo mission (2020, this journal)

J. Gouman, T. Beck, M. Affolter, N. Thomas, U. Geissbühler, A. Péteut, T. Bandy, A. Servonet, D. Piazza, K. Seiferlin, K. Ghose, Measurement and stability of the pointing of the BepiColombo Laser Altimeter under thermal load. Acta Astronaut. 105, 17 (2014)

K. Gunderson, N. Thomas, BELA receiver performance modeling over the BepiColombo mission lifetime. Planet. Space Sci. 58, 309 (2010)

K. Gunderson, N. Thomas, M. Rohner, A Laser Altimeter performance model and its application to BELA. IEEE Trans. Geosci. Remote Sens. 44, 3308 (2006)

M.A. Hofton, J.B. Minster, J.B. Blair, Decomposition of laser altimeter waveforms. IEEE Trans. Geosci. Remote Sens. 38, 1989 (2000)

A. HosseiniArani, BepiColombo Laser Altimeter (BELA) performance evaluation: from laboratory tests to simulations of flight observations. PhD thesis, University of Bern (2020)

A. HosseiniArani et al., Comprehensive in-orbit performance evaluation of the BepiColombo Laser Altimeter (BELA). Planet. Space Sci. 195, 105088 (2021). https://doi.org/10.1016/j.pss.2020.105088

H. Hussmann, K. Lingenauber, R. Kallenbach, K. Enya, N. Thomas, L.M. Lara, C. Althaus, H. Araki, T. Behnke, J.M. Castro-Marin, H. Eisenmenger, T. Gerber, H. de la Revilla Miguel, C. Hüttig, K. Ishibashi, J. Jiménez-Ortega, J. Kimura, M. Kobayashi, H.-G. Lötzke, A. Lichopoj, F. Lüdicke, I. MartínezNavajas, H. Michaelis, N. Namiki, H. Noda, J. Oberst, S. Oshigami, R. García, J. Pablo, J. Rodrigo, K. Rösner, A. Stark, G. Steinbrügge, P. Thabaut, S. del Togno, K. Touhara, S. Villamil, B. Wendler, K. Wickhusen, K. Willner, The Ganymede laser altimeter (GALA): key objectives, instrument design, and performance. CEAS Space J. 11(4), 381-390 (2019). https://doi.org/10.1007/s12567-019-00282-8. http://adsabs.harvard.edu/abs/2019CEAS...11..381H

Iess et al., Space Sci. Rev. (2021). this journal

Iofolla et al., Space Sci. Rev. (2021). this journal 
S.G. Jin, S. Arivazhagan, H. Araki, New results and questions of lunar exploration from SELENE, Chang'E1, Chandrayaan-1 and LRO/LCROSS. Adv. Space Res. 52, 285-305 (2013)

JPL, Planetary Data System Standards Reference, JPL D-7669, Part 2, Version 1.12.0, vol. 01, April 01, 2019

R. Kallenbach, E. Murphy, B. Gramkow, M. Rech, K. Weidlich, T. Leikert, R. Henkelmann, B. Trefzger, B. Metz, H. Michaelis, K. Lingenauber, S. DelTogno, T. Behnke, N. Thomas, D. Piazza, K. Seiferlin, Space-qualified laser system for the BepiColombo Laser Altimeter. Appl. Opt. 52, 8732 (2013)

R. Kallenbach, T. Behnke, H. Perplies, R. Henkelmann, M. Rech, U. Geissbühler, A. Péteut, A. Lichopoj, R. Schroedter, H. Michaelis, K. Seiferlin, N. Thomas, J.M. Castro, M. Herranz, L. Lara, Electromagnetic compatibility of transmitter, receiver, and communication port of a space-qualified laser altimeter, in ESA Workshop on Aerospace EMC, Valencia, Spain, 23-25 May 2016 (IEEE Press, New York, 2016). https://doi.org/10.1109/AeroEMC.2016.7504591

W.A. Kleinhans, Skew ray results for condensing light pipes and reflective baffles. Opt. Eng. 15, 2437-2439 (1976)

F. Laforce, Low noise optical receiver using Si APD. Proc. SPIE 7212, 721210 (2009)

E. Mazarico, M.K. Barker, G.A. Neumann, M.T. Zuber, D.E. Smith, Detection of the lunar body tide by the Lunar Orbiter Laser Altimeter. Geophys. Res. Lett. 41, 2282 (2014)

M.E. Perry et al., The low-degree shape of Mercury. Geophys. Res. Lett. 42, 6951-6958 (2015). https://doi. org/10.1002/2015GL065101

W. Riede, P. Allenspacher, L. Jensen, Analysis of the air-vacuum effect in dielectric coatings. Proc. SPIE 7132, 71320F (2008)

W. Riede, H. Schroeder, P. Allenspacher, P. Mahnke, Aspects of laser optics qualification for space applications. Proc. SPIE 7504, 75040T (2009)

W. Riede, H. Schroeder, G. Bataviciute, D. Wernham, A. Tighe, F. Pettazzi, J. Alves, Laser-induced contamination on space optics. Proc. SPIE 8190, 81901E (2011)

M.S. Robinson, P.G. Lucey, Recalibrated Mariner 10 color mosaics: implications for mercurian volcanism. Science 275, 197 (1997)

J. Rodrigo, E. Gasquet, J.-M. Castro, M. Herranz, L.-M. Lara, M. Muñoz, A. Simon, T. Behnke, N. Thomas, The BepiColombo Laser Altimeter (BeLA) power converter module (PCM): concept and characterisation. Rev. Sci. Instrum. 88, 034702 (2017)

T.S. Rose, M.S. Hopkins, R.A. Fields, Characterization and control of gamma and proton radiation effects on the performance of Nd: YAG and Nd: YLF lasers. IEEE J. Quantum Electron. 31, 1593-1602 (1995)

D.A. Rothery et al., Rationale for BepiColombo studies of Mercury's surface and composition. Space Sci. Rev. 216, 66 (2020, this journal). https://doi.org/10.1007/s11214-020-00694-7

K. Seiferlin, S. Chakraborty, K. Gunderson, J. Fischer, B. Lüthi, D. Piazza, M. Rieder, M. Sigrist, N. Thomas, T. Weigel, Design and manufacture of a lightweight reflective baffle for the BepiColombo Laser Altimeter. Opt. Eng. 46, 043003 (2007)

D.E. Smith, M.T. Zuber, G.A. Neumann, F.G. Lemoine, Topography of the Moon from the Clementine lidar. J. Geophys. Res. 102, 1591 (1997)

D.E. Smith, M.T. Zuber, G.B. Jackson, J.F. Cavanaugh, G.A. Neumann, H. Riris, X. Sun, R.S. Zellar, C. Coltharp, J. Connelly, R.B. Katz, I. Kleyner, P. Liiva, A. Matuszeski, E.M. Mazarico, J.F. McGarry, A.M. Novo-Gradac, M.N. Ott, C. Peters, L.A. Ramos-Izquierdo, L. Ramsey, D.D. Rowlands, S. Schmidt, V.S. Scott, G.B. Shaw, J.C. Smith, J.-P. Swinski, M.H. Torrence, G. Unger, A.W. Yu, T.W. Zagwodzki, The Lunar Orbiter Laser Altimeter investigation on the lunar reconnaissance orbiter mission. Space Sci. Rev. 150, 209 (2010)

D.E. Smith, M.T. Zuber, G.A. Neumann, E. Mazarico, F.G. Lemoine, J.W. Head, P.G. Lucey, O. Aharonson, M.S. Robinson, X. Sun, M.H. Torrence, M.K. Barker, J. Oberst, T.C. Duxbury, D. Mao, O.S. Barnouin, K. Jha, D.D. Rowlands, S. Goossens, D. Baker, S. Bauer, P. Gläser, M. Lemelin, M. Rosenburg, M.M. Sori, J. Whitten, T. Mcclanahan, Summary of the results from the lunar orbiter laser altimeter after seven years in lunar orbit. Icarus 283, 70 (2017)

S.C. Solomon, R.L. McNutt, R.E. Gold, M.H. Acuña, D.N. Baker, W.V. Boynton, C.R. Chapman, A.F. Cheng, G. Gloeckler, J.W. Head III., S.M. Krimigis, W.E. McClintock, S.L. Murchie, S.J. Peale, R.J. Phillips, M.S. Robinson, J.A. Slavin, D.E. Smith, R.G. Strom, J.I. Trombka, M.T. Zuber, The MESSENGER mission to Mercury: scientific objectives and implementation. Planet. Space Sci. 49(14-15), 1445-1465 (2001). https://doi.org/10.1016/S0032-0633(01)00085-X. http://adsabs.harvard.edu/abs/2001P\&SS...49.1445S

O.N. Stavroudis, System of reflective telescope baffles using conic sections of revolution. US Patent 5,255,931 (1993)

G. Steinbrügge, A. Stark, H. Hussmann, K. Wickhusen, J. Oberst, The performance of the BepiColombo Laser Altimeter (BELA) prior launch and prospects for Mercury orbit operations. Planet. Space Sci. 159, 84 (2018) 
N. Thomas, T. Beck, S. Chakraborty, M. Gerber, S. Graf, D. Piazza, G. Roethlisberger, A wide-beam continuous solar simulator for simulating the solar flux at the orbit of Mercury. Meas. Sci. Technol. 22, 065903 (2011)

N. Thomas, H. Hussmann, L.M. Lara, The BepiColombo Laser Altimeter (BELA): a post-launch summary. CEAS Space J. 11(4), 371-380 (2019). https://doi.org/10.1007/s12567-019-00270-y. http://adsabs.harvard.edu/abs/2019CEAS...11..371T

R.N. Thor et al., Determination of the lunar body tide from global laser altimetry data. J. Geod. 95(1), 4 (2021). https://doi.org/10.1007/s00190-020-01455-8

S.-i. Watanabe, Y. Tsuda, M. Yoshikawa, S. Tanaka, T. Saiki, S. Nakazawa, Hayabusa2 mission overview. Space Sci. Rev. 208, 3 (2017)

K. Weidlich, M. Rech, R. Kallenbach, Qualification testing of the laser transmitter part for ESA's BepiColombo Laser Altimeter (BELA), in Proc. SPIE, vol. 8159, Lidar Remote Sensing for Environmental Monitoring XII, 815906 (2011). https://doi.org/10.1117/12.893071

M.T. Zuber, D.E. Smith, S.C. Solomon, D.O. Muhleman, J.W. Head, J.B. Garvin, J.B. Abshire, J.L. Bufton, The Mars Observer Laser Altimeter investigation. J. Geophys. Res. 97, 7781 (1992)

M.T. Zuber, D.E. Smith, R.J. Phillips, S.C. Solomon, G.A. Neumann, S.A. Hauck, S.J. Peale, O.S. Barnouin, J.W. Head, C.L. Johnson, F.G. Lemoine, E. Mazarico, X. Sun, M.H. Torrence, A.M. Freed, C. Klimczak, J.-L. Margot, J. Oberst, M.E. Perry, R.L. McNutt, J.A. Balcerski, N. Michel, M.J. Talpe, D. Yang, Topography of the northern hemisphere of Mercury from MESSENGER Laser Altimetry. Science 336, 217 (2012) 